\title{
SCHUR MULTIPLIERS OF FINITE SIMPLE GROUPS OF LIE TYPE
}

\author{
BY
}

ROBERT L. GRIESS, JR.

ABSTRACT. This paper presents results on Schur multipliers of finite groups of Lie type. Specifically, let $p$ denote the characteristic of the finite field over which such a group is defined. We determine the p-part of the multiplier of the Chevalley groups $G_{2}(4), G_{2}(3)$ and $F_{4}(2)$; the Steinberg variations; the Ree groups of type $F_{4}$ and the Tits simple group ${ }^{2} F_{4}(2)$ '.

Introduction. For a general account of work on the Schur multipliers of the known finite simple groups, the reader is referred to [8]. The gaps in the multiplier situation as discussed in [8] have since been filled (specifically, $m(.1)=2$, $m(.2)=1, m\left(M(24)^{\prime}\right)=1$ ) and these results will appear in a paper dealing with multipliers of sporadic simple groups [9].

In this paper we prove the following theorems about multipliers of groups of Lie type. Theorem 1 will follow from the theorems of Chapter I, the statements of which contain detailed information about generators and relations for the (unique) covering groups of these groups. The results of Chapter II establish Theorems 2 and 3, and the results of Chapter III establish Theorems 4 and 5.

Theorem 1. $m_{2}\left(G_{2}(4)\right)=2, m_{3}\left(G_{2}(3)\right)=3, m_{2}\left(F_{4}(2)\right)=2$.

Theorem 2. $M\left({ }^{2} A_{2}(q)\right) \cong Z(\operatorname{SU}(3, q))$; i.e., $m(\mathrm{SU}(3, q))=1$.

Theorem 3. Let $G$ be a Steinberg variation defined over a finite field of characteristic $p$, i.e., $G={ }^{2} A_{n}(q), n \geq 2,{ }^{2} D_{n}(q), n \geq 4,{ }^{3} D_{4}(q)$ or ${ }^{2} E_{6}(q)$, where $q$ is a power of $p$. Then $M_{p}(G)=1$ except for

$$
\begin{array}{ll}
M_{2}\left({ }^{2} A_{3}(2)\right) \cong Z_{2}, & M_{3}\left({ }^{2} A_{3}(3)\right) \cong Z_{3} \times Z_{3}, \\
M_{2}\left({ }^{2} A_{5}(2)\right) \cong Z_{2} \times Z_{2}, & M_{2}\left({ }^{2} E_{6}(2)\right) \cong Z_{2} \times Z_{2} .
\end{array}
$$

Theorem 4. If $G$ is a Ree group of type $F_{4}$, then $m(G)=1$.

Theorem 5. The Tits simple group ${ }^{2} \mathrm{~F}_{4}(2)^{\prime}$ has trivial multiplier.

Keceived by the editors December 30, 1971.

AMS (MOS) subject classifications (1970). Primary 20C25, 20D05, 20G 40; Secondary 20G05, 2CF 25.

Key words and phrases. Schur multiplier, covering group, finite group of Lie type, simple group, stable cocycle class. 
Most group theoretic notation used here is fairly standard; see [7] or [10]. Notation for groups of Lie type used here is that of [7, p. 491], and [4]. Other notation for classical groups is that found in [10]. Some notation special to this paper is the following:

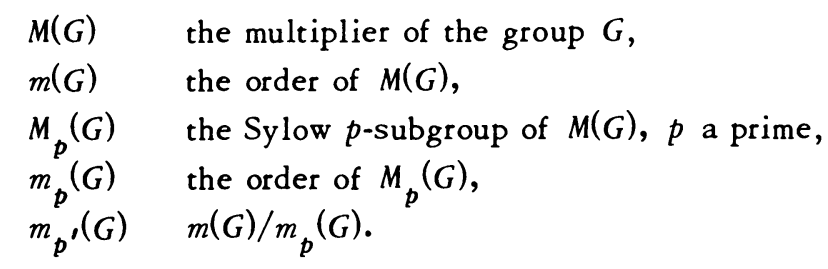

Also, for group elements $x, y$ we define $x^{y}=y^{-1} x y$ and $[x, y]=x^{-1} y^{-1} x y$.

Assumed results. Most of these may be found in [5] or [8]; if not, a source is noted. Fundamental results about multipliers and covering groups are nicely presented in [10].

(1) (Gaschütz's Theorem) If $G$ is a finite group, $H$ a subgroup, $M$ a finite $G$ module and $(|M|,|G: H|)=1$, then an extension of $G$ by $M$ splits if the restriction to $H$ is a split extension.

(2) ("Transfer Lemma") If $P$ is a Sylow $p$-subgroup of $G, x$ a p-element in $Z(G)$, then $x \notin P^{\prime}$ implies $x \notin G^{\prime}$.

(3) (Fitting's Lemma) If $A$ is a group of automorphisms of the finite abelian group $M$ and $(|M|,|A|)=1$, then $M=C_{M}(A) \times[M, A]$.

(4) An automorphism of order prime to $p$ on $P$, a $p$-group, is nontrivial if and only if the induced automorphism of $P / \Phi(P)$ is nontrivial.

(5) The terms $G_{i}$ of the lower central series of $G$ satisfy $\left[G_{i}, G_{j}\right] \leq G_{i+j^{*}}$

(6) (Cartan-Eilenberg) For $H \leq G$, the restriction map $H^{n}(G, M) \rightarrow H^{n}(H, M)$, where $p \nmid|G: H|$, induces a monomorphism of the $p$-primary parts of the cohomology groups. The image is the set of stable elements of $H^{n}(H, M)$ with respect to $G$ ([3, Chapter XII]).

(7) If a Sylow $p$-subgroup $P$ of $G$ is elementary abelian of order $p^{2}$, then $p \nmid m(G)$ if the normalizer of $P$ effects a transformation on $P$ of determinant not 1 . (This follows from $[8$, p. 644].)

(8) All covering groups of a perfect group are isomorphic [10].

(9) An automorphism $\alpha$ of a perfect group can be lifted to an automorphism of the covering group.

Proof. (Alperin) Let $1 \rightarrow R \rightarrow F \stackrel{\pi}{\rightarrow} G \rightarrow 1$ be a free presentation of the perfect group $G$. Say the free generators $x_{i}$ of $F$ map to $g_{i}, i=1, \cdots, n$, a set of generators for $G$. Suppose $g_{i}^{\alpha}=b_{i}$. Write $b_{i}$ as a word $w_{i}\left(g_{1}, \cdots, g_{n}\right)$ in the $g_{j}$. Define an endomorphism $\beta: F \rightarrow F$ by $x_{i}^{\beta}=w_{i}\left(x_{1}, \cdots, x_{n}\right)$. Then $\beta \pi=\pi a$. Now, $x \quad \beta$ maps $R$ into itse lf because if a word $v=v\left(x_{1}, \cdots, x_{n}\right) \operatorname{lies}$ in $R, v^{\beta}=v\left(x_{1}^{\beta}, \cdots, x_{n}^{\beta}\right)$ 
goes under $\pi$ to $v\left(x_{1}^{\beta \pi}, \cdots, x_{n}^{\beta \pi}\right)=v\left(x_{1}^{\pi \alpha}, \cdots, x_{n}^{\pi \alpha}\right)=v\left(x_{1}^{\pi}, \cdots, x_{n}^{\pi}\right)^{\alpha}=1$ because $v \in R$ means $v^{\pi}=1$.

Since $G=G^{\prime}, R F^{\prime}=F$. $\beta$ leaves invariant each vertex of the diagram below.

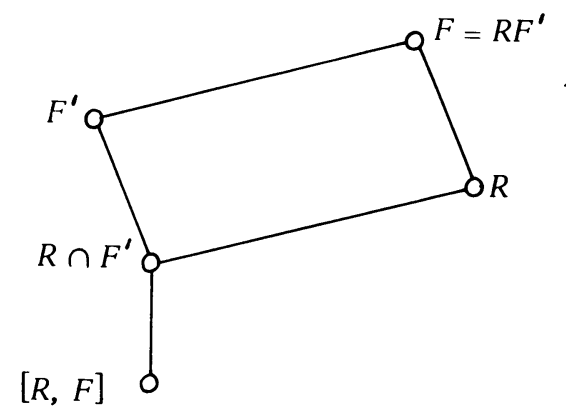

A covering group of $G$ is obtained by taking $F / S$, where $S /[R, F]$ is a complement to $R \cap F^{\prime} /[R, F]$ in $R /[R, F]$. In our case, taking incidence implies $F / S \cong$ $F^{\prime} /[R, F]$ and $\beta$ induces $\alpha$ on $F^{\prime} / R \cap F^{\prime} \cong F / R$. We claim the endomorphism $\beta^{*}$ induced by $\beta$ is an automorphism of $E=F^{\prime} /[R, F]$. Clearly the product of the image of $\beta^{*}$ on $E$ with $R \cap F^{\prime} /[R, F]$ is $E$. But since $R \cap F^{\prime} /[R, F]$ is central, it lies in the Frattini subgroup of $E$. Hence $\beta^{*}$ is onto and so an is omorphism.

(10) If $K / A \cong G, A \leq Z(K) \cap K^{\prime}$, then there is a covering group $H$ of $G$ with quotient isomorphic to $K[10]$.

(11) If $H \triangleleft G, H=H^{\prime}$, and $m_{p}(G / H)=1$, then $m_{p}(H)=1$ implies $m_{p}(G)=1$.

Proof. Take $\widetilde{G}$, a central extension of $G$ by a $p$-group $A$. Then $\widetilde{H}=\widetilde{H}^{\prime} \times A \cong$ $H \times A$ and each factor is normal in $G$. Let $G^{*}=\tilde{G} / \widetilde{H}^{\prime}$, a central extension of $G / H$. If $A^{*}=A \tilde{H}^{\prime} / \tilde{H}^{\prime}, m_{p}(G / H)=1$ implies $A^{*} \cap G^{* \prime}=1$. As $\tilde{G}$ is arbitrary, we get $m_{p}(G)=1$ by $(10)$.

(12) If $K / A=G, A \leq Z(K) \cap K^{\prime}$ and the ordinary representations of $K$ over an algebraically closed field $k$ of characteristic $p>0$ lift the projective representations of $G$ over $k$, then $A \cong M(G) / M_{p}(G)$ (e.g., 3.2 of [18]).

(13) $[x y, z]=[x, z]^{y}[y, z],[x, y z]=[x, z][x, y]^{z}$.

(14) Let $A, B$ be subgroups of $G$. Suppose $[A, B]$ centralizes $A$ and $B$. Then $\left[a a^{\prime}, b\right]=[a, b]\left[a^{\prime}, b\right]$ and $\left[a, b b^{\prime}\right]=\left[a, b^{\prime}\right][a, b], a, a^{\prime} \in A, b, b^{\prime} \in B$. (We say here that $[$,$] is "biadditive" or "bimultiplicative".)$

\section{CHAPTER I. SOME CHEVALLEY GROUPS}

Groups of type $G_{2}$. We begin with a discussion of groups of type $G_{2}$ defined over any finite field $K$. Eventually, we will specialize to $K=\mathrm{GF}(4)$ and $K=\mathrm{GF}(3)$.

Suppose $\Sigma$ is a root system of type $G_{2}$. We may depict $\Sigma$ as a subset of the plane with a real inner product $($,$) .$ 


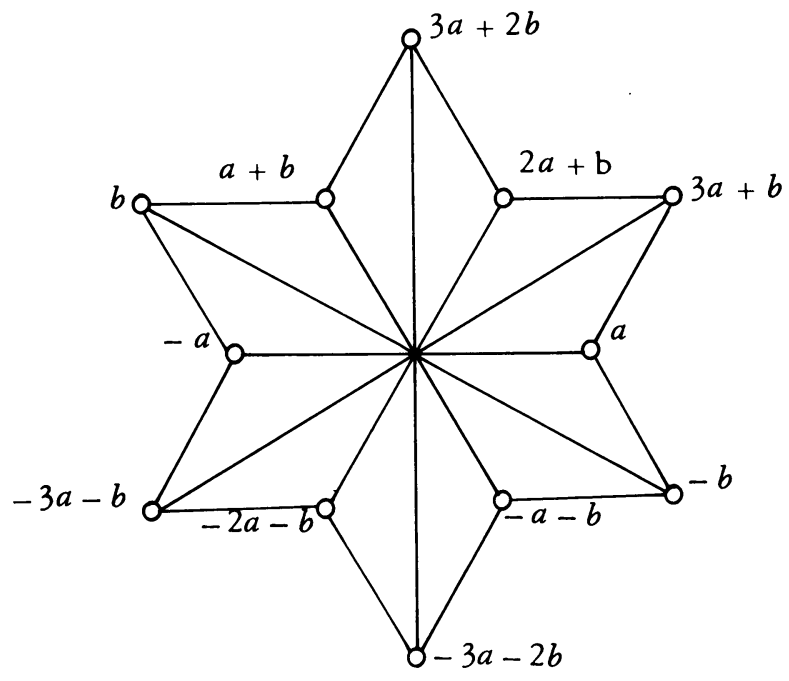

Here, the fundamental roots are $a$ and $b$, and the positive roots $\Sigma^{+}$listed in the usual ordering are $\{a, b, a+b, 2 a+b, 3 a+b, 3 a+2 b\}$.

The group $G_{2}(K)$, defined over the field $K$, is generated by the elements $x_{r}(t), r \in \Sigma, t \in K$. Among the important relations satisfied by these elements are

(A) Additivity. $x_{r}(t) x_{r}(u)=x_{r}(t+u), t, u \in K$, all $r \in \Sigma$.

(B) Chevalley commutator relations. $\left[x_{r}(t), x_{s}(u)\right]=\Pi x_{i r+j s}\left(c_{i j r s} t^{i} u^{j}\right), t$, $u \in K$, all $r, s \in \Sigma, r \not-s$. The $c_{i j r s}$ are certain integers independent of $t$ and $u$, and the product extends over all $i, j$ such that $i r+j s$ is a root, the terms being arranged lexicographically.

We list the commutator relations for $r, s \in \Sigma^{+}$.

$$
\begin{aligned}
{\left[x_{a}(t), x_{b}(u)\right] } & =x_{a+b}(-t u) x_{2 a+b}\left(-t^{2} u\right) x_{3 a+b}\left(t^{3} u\right) x_{3 a+2 b}\left(-2 t^{3} u^{2}\right), \\
{\left[x_{a}(t), x_{a+b}(u)\right] } & =x_{2 a+b}(-2 t u) x_{3 a+b}\left(3 t^{2} u\right) x_{3 a+2 b}\left(3 t u^{2}\right), \\
{\left[x_{a}(t), x_{2 a+b}(u)\right] } & =x_{3 a+b}(3 t u), \\
{\left[x_{b}(t), x_{3 a+b}(u)\right] } & =x_{3 a+2 b}(t u), \\
{\left[x_{a+b}(t), x_{2 a+b}(u)\right] } & =x_{3 a+2 b}(3 t u) .
\end{aligned}
$$

All other commutators $\left[x_{r}(t), x_{s}(u)\right], r, s \in \Sigma^{+}, t, u \in K$, are trivial. These formulas are derived from $[12, \mathrm{p} .443]$.

Let $K=\mathrm{GF}(q)$, where $q$ is a power of the prime $p$. If we let $U=\left\langle x_{r}(t)\right| r \in \Sigma+$, $t \in K\rangle$, then $U$ is a Sylow p-subgroup of $G=G_{2}(q)$. Also, $N_{G}(U)=U H$, where $H$ is a Cartan subgroup. We have $H=\left\langle b_{a}(\omega)\right\rangle \times\left\langle b_{b}(\omega)\right\rangle$, where $\omega$ generates $K^{\times}$and each factor is isomorphic to $Z_{q-1}$. In fact, $b_{r}(u) b_{r}(v)=b_{r}(u v)$, for $u, v \in K^{\times}, r=a, b$. For each $r \in \Sigma$, $H$ normalizes $X_{r}=\left\langle x_{r}(t) \mid t \in K\right\rangle$, and $x_{r}(t)^{b_{s}(u)}=x_{r}\left(t u^{c}\right)$, where $c$ is the Cartan integer $c=c(r, s)=2(r, s) /(s, s)$. 
Notice that certain terms in the relations of type (B) drop out in characteristic 2 or 3. As usual, we let $U_{i}$ be the $i$ th member of the descending central series of $U$. When $K=\mathrm{GF}(4)$, we have

$$
\begin{aligned}
& U_{1}=U, \quad U_{2}=\left\langle X_{a+b}, X_{2 a+b}, X_{3 a+b}, X_{3 a+2 b}\right\rangle, \\
& U_{3}=\left\langle X_{3 a+b}, X_{3 a+2 b}\right\rangle, \quad U_{4}=\left\langle X_{3 a+2 b}\right\rangle, \quad U_{5}=1 .
\end{aligned}
$$

In fact, reversing the order of the terms will give the upper central series. So, the class of $U$ is four, $U_{i} / U_{i+1}$ is elementary abelian, $1 \leq i \leq 4$, of order $2^{4}, 2^{4}, 2^{2}$, $2^{2}$, respectively. When $K=\mathrm{GF}(3)$, we have

$$
\begin{aligned}
& U_{1}=U, \quad U_{2}=\left\langle X_{2 a+b}, X_{3 a+2 b}, x_{a+b}(v)_{3 a+b}(-v) \mid v \in K\right\rangle, \\
& U_{3}=\left\langle X_{2 a+b}, X_{3 a+2 b}\right\rangle, \quad U_{4}=1 .
\end{aligned}
$$

The factor groups $U_{i} / U_{i+1}, 1 \leq i \leq 3$, are elementary abelian of orders $3^{3}, 3,3^{2}$, respectively.

The Weyl group of this root system is dihedral of order 12 and acts as the group of symmetries of a hexagon on the set of short roots and on the set of long roots.

Denote by $H$ a Cartan subgroup of $G$. Assume $q \neq 2$. If we set $N=N_{G}(H)$, then $N / H \cong W$, the Weyl group of $\Sigma$. For each $w_{r}$, the fundamental reflection through the hyperplane orthogonal to $r \in \Sigma$, there is a distinguished $H$-coset representative $n_{r}$ of $N$ which maps onto $w_{r}$ under the above homomorphism: $n_{r}=$ $x_{r}(-1) x_{-r}(1) x_{r}(-1)$. If $q=2$, we define the subgroup $N$ as the subgroup generated by all the $n_{r}$. This element $n_{r}$ has the property $x_{s}(t)^{n_{r}}=x_{w_{r}}(s)\left(\eta_{r, s} t\right)$, where the $\eta_{r, s}$ are certain integers \pm 1 . These $\eta_{r, s}$ satisfy the rules $\eta_{r, r}=-1, \eta_{r,-s}=$ $\eta_{r, s}, \eta_{-r, s}=\eta_{r, w_{r}(s)}:$ A table of $\eta_{r, s}$ for $r, s \in \Sigma^{+}$, taken from Ree, is given below [12].

\section{Table 1}

$$
\text { s: } \quad a \quad b \quad a+b \quad 2 a+b \quad 3 a+b \quad 3 a+2 b
$$

$r$ :

$\begin{array}{lrrrrrr}a & -1 & -1 & -1 & 1 & 1 & 1 \\ b & -1 & -1 & 1 & 1 & -1 & 1 \\ a+b & 1 & 1 & -1 & -1 & 1 & -1 \\ 2 a+b & 1 & 1 & -1 & -1 & 1 & -1 \\ 3 a+b & -1 & -1 & 1 & 1 & -1 & 1 \\ 3 a+2 b & 1 & 1 & 1 & -1 & -1 & -1\end{array}$

If we denote by $G^{*}$ the abstract group presented by generators $x_{r}(t), r \in \Sigma$, 
$t \in K$, satisfying relations (A) and (B), then the theorems of R. Steinberg in [15] show that $G^{*}$ is the covering group of $G$ for representations over an algebraically closed field of characteristic $p$, and that $G^{*} \cong G$. Hence $p$ is the only prime which can divide the order of the Schur multiplier of $G$. We shall assume this fact henceforth.

Let $\hat{G}$ be the covering group of $G$. Since $G$ is simple, $\hat{G}=\hat{G}^{\prime}$, and the kernel of the given map $\phi: \hat{G} \rightarrow G$ is $A=Z(\hat{G})$, the center of $\hat{G}$. When convenient we shall regard $\phi$ as the canonical quotient map of $G$ by $A$.

If $V$ denotes the preimage of $U$ in $\hat{G}$, then $V$ is a Sylow $p$-subgroup of $G$ and $V / A=U$. We have $\left[V_{i}, V_{j}\right] \leq V_{i+j}$, as is true in any p-group, but also $\left[A V_{i}, A V_{j}\right] \leq$ $V_{i+j}$, because $A$ is central. Note further that $[x, y]$, for any $x, y \in \hat{G}$, depends only on the coset of each argument modulo the center $A$.

The group $G_{2}(4)$.

Theorem. The Scbur multiplier of $G=G_{2}(4)$ bas order 2. The covering group $\hat{G}$ of $G$ may be given with the following generators and relations:

$(\hat{A})$

$$
\begin{aligned}
\left\{y_{r}(t) \mid r \in \Sigma, t \in K\right\}, & K=\mathrm{GF}(4), \quad K^{\times}=\left\{1, \omega, \omega^{2}\right\} ; \\
& \sum \text { is a root system of type } G_{2} . \\
y_{r}(0)=1, & y_{r}^{\prime}(t)^{2}=\zeta,
\end{aligned}
$$

$$
\begin{aligned}
y_{r}(1) y_{r}(\omega)=y_{r}\left(\omega^{2}\right), \quad y_{r}(t) y_{r}(u) & =y_{r}(u) y_{r}(t) \zeta, \\
& \text { for all short roots } r \in \Sigma, t, u \in K^{\times} ;
\end{aligned}
$$

$$
y_{r}(t) y_{r}(u)=y_{r}(t+u), \text { all } t, u \in K \text {, all long roots } r \in \Sigma \text {, }
$$

$$
\left[y_{r}(t), y_{s}(u)\right]=\prod_{i, j} y_{i r+j s}\left(c_{i j r s} t^{i} u^{j}\right) f_{r, s}(t, u)
$$

where $f_{r, s}(t, u)=1$ unless $r$ is short (resp. long), $s$ is long (resp. short), and they form an angle of $150^{\circ}$, in which case $f_{r, s}(t, u)=\zeta$ for $t u \neq 0$, or unless $r$ and $s$ are long inclined $120^{\circ}$ to each other, and $t \neq u, t u \neq 0$ in which case $f_{r, s}(t, u)=\zeta$; the $c_{i j \mathrm{rs}}$ are the same integers as in the corresponding formula in $G_{2}(4)$, and the terms in the product are ordered correspondingly.

We are interested in a set of representatives $\widetilde{x_{r}(t)} \in \hat{G}$ and relations like (A) and (B) holding among them. Let $Y_{r}$ be the preimage of $X_{r}$ in $G ; Y_{r} / A=X_{r} \cdot \operatorname{Re}-$ call that $V$ is the preimage of $U$ in $G$ and that $V_{i}$ denotes the $i$ th term of the descending central series of $V$.

Lemma 1. $Y_{r}$ is abelian if $r$ is long. If $s$ is short, $Y_{s}^{\prime}=\langle\zeta\rangle, \zeta^{2}=1$, for all short roots $s$. 
Proof. For some $w \in W,\left(X_{r}\right)^{n_{w}}=X_{3 a+2 b^{*}}$ So, $Y_{r}^{\prime} \subseteq\left[A V_{4}, A V_{4}\right] \subseteq V_{8}=1$, and $Y_{r}$ is abelian.

Let $f_{s}(t, u)=\left[\tilde{x}_{s}(t), \tilde{x}_{s}(u)\right]$. By applying the $n_{w}, w \in W, f_{s}(t, u)=f_{a}(t, u)$ for all short roots $s$. Conjugating the commutator by $b_{a}(v)$, we get $f_{a}(t, u)=$ $f_{a}\left(t v^{2}, u v^{2}\right), v \in K^{\times}$. Also, $1=\left[\tilde{x}_{a}(t), \tilde{x}_{a}(u)^{2}\right]=f(t, u)^{2}$, so $f(t, u)=f(u, t)$ as $[x, y]^{-1}=[y, x]$. All this implies $f_{a}(t, u)=f_{a}\left(t^{\prime}, u^{\prime}\right)$ for any $t \neq u, t^{\prime} \neq u^{\prime}$ in $K^{\times}$. Setting $\zeta=f_{a}(t, u)$ for $t \neq u$ finishes the proof.

For $r$ long, Fitting's theorem gives us a decomposition $Y_{r}=\left[Y_{r}, H\right] \oplus A$. Define $y_{r}(t)$ as the unique element of $x_{r}(t) \cap\left[Y_{r}, H\right]$. For $s$ short, define $y_{s}(0)=1$ and $y_{s}(\lambda t)=\left[\tilde{x}_{s}(t), b_{s}(\lambda)\right]$, for $t \neq 0$ where $\langle\lambda\rangle=K^{\times}$. In either case, these representatives satisfy

$$
\begin{array}{rlrl}
y_{r}(t)^{b} & =y_{r}\left(t^{\prime}\right) & \text { where } b \in H, \quad x_{r}(t)^{b}=x_{r}\left(t^{\prime}\right), \\
y_{r}(t)^{n_{w}} & =y_{w(r)}(t) \quad \text { for } n_{w} \in N, \quad x_{r}(t)^{n_{w}}=x_{w(r)}(t), \\
y_{r}(t) y_{r}(u) & =y_{r}(t+u) & \text { for } r \text { long, } t, u \in K, \\
y_{s}(t) y_{s}(u) & =y_{s}(t+u) d_{s}(t, u) & \text { for } s \text { short, } t, u \in K, d_{s}(t, u) \in A, \\
y_{s}(t)^{2} & =\zeta & \text { for } s \text { short, } t \neq 0 .
\end{array}
$$

The last fact comes from $1=\left[\tilde{x}_{s}(t)^{2}, b_{s}(\lambda)\right]=y_{s}(\lambda t)^{2} \zeta$.

The factor set $d(t, u)=d_{s}(t, u)$ is independent of the short root $s$, by the above. In the group $\bar{G}=\hat{G} /\langle\zeta\rangle, \bar{Y}_{s}$ is abelian. So, Fitting's theorem applies to give $X_{s}=\left[\bar{Y}_{s}, H\right]=\left\langle\bar{y}_{s}(t) \mid t \in K\right\rangle$, and then $\bar{y}_{s}(t) \bar{y}_{s}(u)=\bar{y}_{s}(t+u)$. This says that $d(t, u) \in\langle\zeta\rangle$. As in the previous lemma, $d(t, u)=d(t v, u v)$, all $v \in K^{\times}$. Clearly, $d(t, u)=\zeta d(u, t)$.

The relations (B). For the analogue of a typical Chevalley commutator relation in $\hat{G}$ we write

$$
\left[y_{r}(t), y_{s}(u)\right]=\prod y_{i r+j s}\left(c_{i j r s} t^{i} u^{j}\right) f_{r, s}(t, u) .
$$

Our object is to describe the $f_{r, s}(t, u)$. By applying some $n_{w}$, we then may assume $r, s$ is any given pair of roots of the same lengths forming the same angles, as $W$ is transitive on such pairs.

We can now give a description of certain $f_{r, s}(t, u), r \neq s,-s$.

Lemma 2. Suppose that $X_{r}$ and $X_{s}$ bave different centralizers in $H$ and that $\left[y_{r}(t), y_{s}(u)\right]=\Pi y_{i r+j s}\left(c_{i j r s} t^{i} u^{j}\right) f_{r, s}(t, u)$. Then,

$$
f_{r, s}(t, u)=f_{r, s}\left(t_{1}, u_{1}\right) \text {, for all } t, u, t_{1}, u_{1} \in K^{\times} \text {. }
$$

Moreover, if $\left[y_{r}(t), y_{s}(u)\right]$ commutes with $y_{r}(t)$ and $y_{r}(u)$, and if the $y_{i r+j s}()$ which appear are additive in their arguments and commute with each other, then $f_{r, s}(t, u) \equiv 1$. 
Proof. No $X_{r}$ is centralized by all of $H$, so no $Y_{r}$ is either. By our convention, $y_{r}(0)=1$, all $r^{\prime} \in \Sigma$. So, conjugate the commutator equation first by each $b \in C_{H}\left(X_{r}\right)$ and secondly by every $b \in C_{H}\left(X_{s}\right)$ to obtain the first result. The last hypothesis implies that $[$,$] and all the y_{i r+j s}()$ are bilinear functions of the commutator arguments. This, with the first result, proves $f_{r, s}(t, u) \equiv 1$.

Corollary 1. $\left[y_{r}(t), y_{s}(u)\right]=1$ whenever $\left[x_{r}(t), x_{s}(u)\right]=1$ unless $r$ and $s$ are long roots forming an angle of $60^{\circ}$.

Proof. To use the lemma we must check $C_{H}\left(X_{r}\right) \neq C_{H}\left(X_{s}\right)$ in all the above cases. Since $r \neq s$, there is some $w \in W$ for which $w(r), w(s) \in \Sigma^{+}$. So, $\left[X_{r}, X_{s}\right]=$ $1, C_{H}\left(X_{r}\right) \neq C_{H}\left(X_{s}\right)$ is equivalent to $\left[X_{w(r)}, X_{w(s)}\right]=1, C_{H}\left(X_{w(r)}\right) \neq C_{H}\left(X_{w(s)}\right)$, which is checked for the relevant positive roots by inspecting the Cartan integers.

A separate argument disposes of the exceptional case.

Lemma 3. If $r, s \in \Sigma$ are long roots forming an angle of $60^{\circ}$, then $\left[y_{r}(t)\right.$, $\left.y_{s}(u)\right]=1$.

Proof. $y_{r}(t), y_{s}(u)$ are conjugate to, respectively, $y_{3 a+2 b}(t)$ modulo $A$ and $y_{3 a+b}(u)$ modulo $A$, by some $n_{w} \in N, w \in W$. The commutator is central, so $\left[y_{r}(t), y_{s}(u)\right]=\left[y_{3 a+2 b}(t), y_{3 a+b}(u)\right] \in\left[A V_{4}, A V_{3}\right] \subseteq V_{7}=1$, as $V$ has nilpotence class 4 or 5 .

Corollary 2. If $s$ and $r$ are short roots, inclined $60^{\circ}$ to each other then $\left[y_{s}(t)\right.$, $\left.y_{r}(u)\right]=y_{s+r}(t u)\left(s+r\right.$ is long). If $s$ and $r$ are short roots inclined $120^{\circ}$ to each other, then $\left[y_{s}(t), y_{r}(u)\right]=y_{2 s+r}\left(t^{2} u\right) y_{s+2 r}\left(t u^{2}\right)(2 s+r$ and $s+2 r$ are long roots $)$.

Proof. In the first case, by the conjugacy properties of the $y_{r}(t)$ under the $n_{w}$, we may assume $s=a+b, r=2 a+b, s+r=3 a+2 b$. So, we have that $\left[y_{a+b}(t)\right.$,

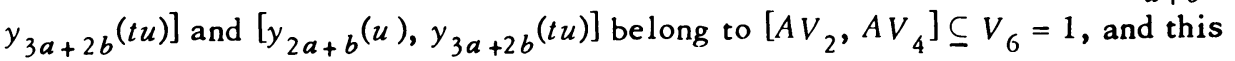
gives $f_{a+b, 2 a+b}(t, u) \equiv 1$, using Lemma 2 .

In the second case, we may assume, as above, that $s=a, r=a+b, 2 s+r=$ $3 a+b, s+2 r=3 a+2 b$. Arguing as above, we apply Lemma 2 to finish the proof.

Lemma 4. If $s, r$ are long roots forming an angle of $120^{\circ},\left[y_{s}(t), y_{r}(u)\right]=$ $y_{s+r}(t u) f_{s, r}(t, u)$. Then $f_{s, r}(t, u)=1$ if $t=0, u=0, t=u$. For other $t, u$, $f_{s, r}(t, u)$ assumes the constant value $\gamma, \gamma^{2}=1$.

Proof. We may assume $s=b, r=3 a+b, s+r=3 a+2 b .\left[X_{b}, X_{3 a+2 b}\right]=$ $\left[X_{3 a+b}, X_{3 a+2 b}\right]=1$ implies that $f_{b, 3 a+b}(t, u)$ is bilinear. Since $C_{H}\left(X_{b}\right)=$ $C_{H}\left(X_{3 a+b}\right), f_{b, 3 a+b}(t v, u v)=f_{b, 3 a+b}(t, u)$, all $v \in K^{\times}$. Application of $n_{a}$ to the commutator equation shows that $f_{b, 3 a+b}(t, u)=f_{b, 3 a+b}(u, t)$. All these facts prove the various parts of the lemma.

Lemma 5. For $\left[y_{r}(t), y_{s}(u)\right]=y_{r+s}(t u) f_{r, s}(t, u)$, where $r$ and $s$ are long roots 
forming a $120^{\circ}$ angle, $f_{r, s}(t, u)=1$ for $t$ or $u=0$ or $t=u, f_{r, s}(t, u)=\delta$ otherwise, some $\delta \in A$ independent of the particular $r, s$.

Proof. By previous relations, $f_{r, s}$ is biadditive. Conjugating the relation by $b_{r}(v), f_{r, s}(t, u)=f_{r, s}\left(v^{2} t, v^{-1} u\right)=f_{r, s}\left(v^{2} t, v^{2} u\right), v \in K^{\times}$. Conjugating by $n_{t}$, where $t$ is a short root orthogonal to $r+s, f_{r, s}(t, u)=f_{s, r}(t, u)=f_{r, s}(u, t)$. If $0 \neq t \neq u \neq 0$, we get $f_{r, s}(t, u)=\delta$. Let $0 \neq t=u+v$, where $u \neq 0 \neq v$. Then $f_{r, s}(t, t)=f_{r, s}(t, u) f_{r, s}(t, u)=\delta^{2}=1$. Conjugating the commutator by all $n_{w}$, $w \in W$, we prove the last part.

Lemma 6. $f_{a, b}(t, u)=\zeta=\delta$ for all $t \neq 0 \neq u$.

Proof. By the first part of Lemma $2, f_{a, b}(t, u)=\gamma$ for all $t u \neq 0$. Now,

$$
\begin{aligned}
& y_{a}(t) y_{a+b}\left(t\left(u_{1}+u_{2}\right)\right) y_{2 a+b}\left(t^{2}\left(u_{1}+u_{2}\right)\right) y_{3 a+b}\left(t^{3}\left(u_{1}+u_{2}\right)\right) f_{a, b}\left(t, u_{1}+u_{2}\right) \\
& =y_{a}(t)^{y_{b}\left(u_{1}+u_{2}\right)}=\left\{y_{a}(t) y_{a+b}\left(t u_{1}\right) y_{2 a+b}\left(t^{2} u_{1}\right) y_{3 a+b}\left(t^{3} u_{1}\right) f_{a, b}\left(t, u_{1}\right)\right\}^{y_{b}}\left(u_{2}\right) \\
& =y_{a}(t) y_{a+b}\left(t u_{2}\right) y_{2 a+b}\left(t^{2} u_{2}\right) y_{3 a+b}\left(t^{3} u_{2}\right) f_{a, b}\left(t, u_{2}\right) y_{a+b}\left(t u_{1}\right) \\
& \cdot y_{2 a+b}\left(t^{2} u_{2}\right) y_{3 a+b}\left(t^{3} u_{1}\right) y_{3 a+2 b}\left(t^{3} u_{1} u_{2}\right) f_{3 a+b, b}\left(t^{3} u_{1}, u_{2}\right) f_{a, b}\left(t, u_{1}\right) \\
& =y_{a}(t) y_{a+b}\left(t u_{2}+t u_{1}\right) d\left(t u_{2}, t u_{1}\right) y_{2 a+b}\left(t^{2} u_{2}+t^{2} u_{1}\right) d\left(t^{2} u_{2}, t^{2} u_{1}\right) \\
& \cdot y_{3 a+2 b}\left(t^{3} u_{1} u_{2}\right) y_{3 a+b}\left(t^{3} u_{2}+t^{3} u_{1}\right) y_{3 a+2 b}\left(t^{3} u_{1} u_{2}\right) \\
& \cdot f_{3 a+b, b}\left(t^{3} u_{1}, u_{2}\right) f_{a, b}\left(t, u_{2}\right) f_{a, b}\left(t, u_{1}\right) .
\end{aligned}
$$

Comparing sides,

$$
\begin{aligned}
& f_{a, b}\left(t, u_{1}+u_{2}\right) \\
& \quad=d\left(t u_{2}, t u_{1}\right) d\left(t^{2} u_{2}, t^{2} u_{1}\right) f_{3 a+b, b}\left(t^{3} u_{1}, u_{2}\right) f_{a, b}\left(t, u_{1}\right) f_{a, b}\left(t, u_{2}\right) .
\end{aligned}
$$

(*)

A similar computation with $y_{b}(u)^{y_{a}^{\left(t_{1}+t_{2}\right)}}=\left\{y_{b}(u)^{y_{a}^{\left(t_{1}\right)}}\right\}^{y_{a}^{\left(t_{2}\right)}}$ yields

$$
f_{a, b}\left(t_{1}+t_{2}, u\right)^{-1}=d\left(t_{2}^{2} u, t_{1}^{2} u\right) d\left(t_{2} u, t_{1} u\right) f_{a, b}\left(t_{2}, u\right)^{-1} f_{a, b}\left(t_{1}, u\right)^{-1} \text {. }
$$

Taking $t=1, u_{1}=u_{2} \neq 0$ in (*), we get $1=d\left(u_{1}, u_{1}\right)^{2} \gamma^{2}=\gamma^{2}$. For $t=1$, $0 \neq u_{1} \neq u_{2} \neq 0, \gamma=d\left(u_{2}, u_{1}\right)^{2} \delta \gamma^{2}=\delta$. Taking $u=1,0 \neq t_{1} \neq t_{2} \neq 0$ in (**), $\gamma^{-1}=d\left(t_{1}, t_{1}^{2}\right) d\left(t_{1}^{2}, t_{1}\right) \gamma^{-2}=\zeta$. So, $\gamma=\delta=\zeta$, proving the lemma.

Proof of Theorem. $\hat{G} /\langle\zeta\rangle=G$ because, modulo $\langle\zeta\rangle$, the $y_{r}(t)$ satisfy the relations (A) and (B), which define the group $G$ abstractly. Hence, $|A|=|\zeta|$ divides 2.

The following is a sketch of the proof that 2||$A \mid$. Let $P=\left\langle U H, X_{-a}\right\rangle$ be a maximal parabolic subgroup. In the permutation representation of $G$ on the cosets 
of $P$, one can (by specifying a system of coset representatives) compute that the involution $x_{a}(t)$ fixes 25 cosets out of 1365 . As a permutation, it is the product of $1 / 2(1365-25)=670$ transpositions. If we embed $G$ in the alternating group $A_{1365}$ in this way, and let $\widetilde{G}$ be the preimage of $G$ in the covering group $\hat{A}_{1365}$ of $A_{1365}$, the fact that $670 \equiv 2(\bmod 4)$ means that a representative in $\tilde{G}$ for $x_{a}(t)$ has square $j,\langle j\rangle=Z\left(\hat{A}_{1365}\right)$ [14]. Hence, $\widetilde{G}$ is a nonsplit central extension of $G$ by $\langle j\rangle=Z_{2} . \zeta \neq 1$ and the theorem follows.

Curiously, the representations of $G$ of degree 1365 on the other parabolic subgroup $Q=\left\langle U H, X_{-b}\right\rangle$ leads to a split extension as $x_{a}(t)$ fixes 21 cosets.

The group $G_{2}(3)$. In this section, we prove the following result.

Theorem. The Schur multiplier of $G_{2}(3)$ bas order 3. The (unique) covering group of $G_{2}(3)$ may be presented as the group generated by symbols $y_{r}(t), r \in \Sigma$, a root system of type $G_{2}$, and $t \in K=\mathrm{GF}(3)$, satisfying the relations

$$
\begin{aligned}
y_{r}(t) y_{r}(u) & =y_{r}(t+u), \quad \text { all } r \in \Sigma, t \in K, \\
{\left[y_{r}(t), y_{s}(u)\right] } & =\prod_{i, j} y_{i r+j s}\left(c_{i j r s} t^{i} u^{j}\right) a_{r s}(t, u),
\end{aligned}
$$

where the indices run over the same roots as in the corresponding Chevalley commutator formula, the $c_{i j r s}$ are the same integers, and the terms on the right-hand side are arranged correspondingly. The $a_{r s}(t, u)$ are 1 unless $\{r, s\}$ is a pair of orthogonal roots or a pair of roots at an angle of 150 degrees to one another. If $\{r, s\}$ is an orthogonal pair, then

$$
a_{r, s}(t, u)=a_{w_{s^{\prime}}(r), w_{s^{\prime}}(s)}\left(\eta_{s^{\prime}, r^{\prime}}, \eta_{s^{\prime}, s} u\right)
$$

for all reflections $w_{s^{\prime}} \in W$, the Weyl group of $\Sigma$, where the $\eta_{r, s}$ are certain integers \pm 1 listed later in this paper. If $r$ is a short root and $s$ is a long root forming an angle of $150^{\circ}$, then

$$
a_{r, s}(1,1)=a_{r, s}(t, u)=\alpha_{w(r), w(s)}(t, u)
$$

for all $t, u \in K$ and all $w \in W$. Finally $a_{a, b}(1,1)=a_{a, 3 a+2 b}(1,1)^{-1}$.

Lemma 1. Set $Y_{r} / A=X_{r}$. Then $Y_{r}$ is abelian.

Proof. If $r$ is short (resp. long), then $X_{r}$ is conjugate to $X_{2 a+b}$ (resp. $\left.X_{3 a+2 b}\right)$. Since each of these last two groups lies in $U_{3}$, we have $\left[A V_{3}, A V_{3}\right] \leq$ $V_{6}=1$. The last equality holds because $U$ having class 4 forces $V$ to have nilpotence class 4 or 5 . Thus, $Y_{r}^{\prime}=1$, for all $r$.

We choose a system of generators of $\hat{G}$ as follows. Write $\hat{H} / A=H, \hat{N} / A=N$. Then $\hat{N} / \hat{H} \cong N / H \cong W$. Since $\hat{H}$ induces a 2-group of automorphisms on the abelian 3-group $Y_{r}$, we obtain a Fitting decomposition 


$$
Y_{r}=\left[Y_{r}, \hat{H}\right] \oplus C_{Y_{r}}(H)=\left[Y_{r}, \hat{H}\right] \oplus A \text {. }
$$

This is a direct sum of $\hat{H}$-modules (3). By regarding $X_{r}$ as an $\hat{H}$-module, we have the isomorphism $\left[Y_{r}, H\right] \cong X_{r}$ of $\hat{H}$-modules induced by the quotient map $\phi$. We now select the generators $y_{r}(t)$ from the cosets $x_{r}(t)$ by the rule $y_{r}(t)=[Y, \hat{H}] \cap$ $x_{r}(t)$.

These generators enjoy the following properties, which follow from the definition and the corresponding properties of the $x_{r}(t)$ in $G$.

$$
\begin{aligned}
y_{r}(t) y_{r}(u)=y_{r}(t+u), & \text { all } r \in \Sigma, \text { all } t, u \in K, & \\
y_{r}(t)^{\hat{b}}=y_{r}\left(t^{\prime}\right), & \text { where } \hat{b} \in b \in H, & x_{r}(t)^{b}=x_{r}\left(t^{\prime}\right), \\
y_{r}(t)^{\hat{n}_{w}}=y_{w(r)}( \pm t), & \text { where } \hat{n}_{w} \in n_{w}, w \in W, & x_{r}(t)^{n_{w}}=x_{w(r)}( \pm t) .
\end{aligned}
$$

The relations (B) are satisfied by the $y_{r}(t)$ modulo a factor from $A$. That is, ( $\hat{B})$

$$
\left[y_{r}(t), y_{s}(u)\right]=\prod_{i, j} y_{i r+j s}\left(c_{i j r s} t^{i} u^{j}\right) \alpha_{r s}(t, u)
$$

where the indices run over the same values as in (B), the $c_{i j r s}$ are the same as before, and $\alpha_{r s}(t, u) \in A$. Our task now is to determine these last functions.

Lemma 2. Suppose that $X_{r}$ and $X_{s}$ bave different centralizers in $H$ and that $\left[y_{r}(t), y_{s}(u)\right]=\Pi y_{i r+j s}\left(c_{i j r s} t^{i} u^{j}\right) \alpha_{r s}(t, u)$. Then $\alpha_{r s}(t, u)=\alpha_{r s}\left(t^{\prime}, u^{\prime}\right)$ for all $t, u, t^{\prime}, u^{\prime} \in K^{\times}$. Moreover if $\left[y_{r}(t), y_{s}(u)\right]$ commutes with $y_{r}(t)$ and $y_{s}(u)$ and if the $y_{i r+j s}\left(c_{i j r s} t^{i} u^{j}\right)$ which are not 1 commute with each other, then $\alpha_{r s}(t, u)=1$, for all $t, u$.

Proof. No $X_{r}$ is centralized by all of $H$, so no $Y_{r}$ is centralized by all of $H$. So, conjugate the commutator equation first by an $\hat{b}$ from each $b \in C_{H}\left(X_{r}\right)$ and secondly by every $\hat{b} \in b \in C_{H}\left(X_{s}\right)$ to obtain the first result. The last hypothesis implies that $[$,$] is a bilinear function of its arguments, hence \alpha_{r s}(t, u)$ is. This, with the first result implies $a_{r s}(t, u)=1$.

Corollary. $\alpha_{r s}(t, u)=1$, unless $\{r, s\}$ is conjugate under the Weyl group to eitber $\{a, 3 a+2 b\}$ or $\{a, b\}$.

Proof. Clearly, $\alpha_{r s}(t, u)=\alpha_{w(r), w(s)}( \pm t, \pm u)$, for all $w \in W$. Except for the excluded cases, we apply the lemma to $r, s$ positive. Inspection of the explicit relations (B) and the Cartan integers (below) gives the conclusion.

Table 2

$$
\begin{array}{rrrrccc}
r: & a & b & a+b & 2 a+b & 3 a+b & 3 a+2 b \\
c(r, a): & 2 & -3 & -1 & 1 & 3 & 0 \\
c(r, b): & -1 & 2 & 1 & 0 & -1 & 1
\end{array}
$$


We now deal with the remaining cases.

Lemma 4. $a_{a, b}(1,1) a_{a, 3 a+2 b}(1,1)=1$.

Proof. Conjugate $y_{a}(t)$ by $y_{b}(1)$ twice.

$$
\begin{aligned}
y_{a}(t)^{y_{b}(-1)}=\left\{y_{a}(t) y_{a+b}(-t) y_{2 a+b}\left(-t^{2}\right) y_{3 a+b}\left(t^{3}\right) y_{3 a+2 b}\left(t^{3}\right) a_{a, b}(t, 1)\right\}^{y_{b}(1)} \\
=y_{a}(t) y_{a+b}(-t) y_{2 a+b}\left(-t^{2}\right) y_{3 a+b}\left(t^{3}\right) y_{3 a+2 b}\left(t^{3}\right) a_{a, b}(t, 1) y_{a+b}(-t) \\
\cdot y_{2 a+b}\left(-t^{2}\right) a_{2 a+b, b}\left(-t^{2}, 1\right) y_{3 a+b}\left(t^{3}\right) y_{3 a+2 b}\left(-t^{3}\right) y_{3 a+2 b}\left(t^{3}\right) a_{a, b}(t, 1) \\
=y_{a}(t) y_{a+b}(t) y_{2 a+b}\left(t^{2}\right) y_{3 a+b}\left(-t^{3}\right) y_{3 a+2 b}\left(t^{3}\right) \\
\cdot a_{a, b}(t, 1)^{2} a_{3 a+b, a+b}\left(t^{3},-t\right) a_{2 a+b, b}\left(-t^{2}, 1\right) .
\end{aligned}
$$

Comparing the above with the expression for $y_{a}(t)^{y} b^{(-1)}$ gives the identity

$$
a_{a, b}(t, 1)^{2} \alpha_{3 a+b, a+b}\left(t^{3},-t\right) a_{2 a+b, b}\left(-t^{2}, 1\right)=a_{a, b}(t, 1) .
$$

For an orthogonal pair, bilinearity and the rules

$$
a_{r, s}(t, u)=a_{s, r}(t, u)^{-1}, \quad a_{r, s}(t, u)=a_{w_{s^{\prime}(r)}, w_{s^{\prime}(s)}}\left(\eta_{s^{\prime}, r} t, \eta_{s^{\prime} s^{\prime}} u\right)
$$

imply that, for $t \neq 0, a_{a, b}(t, 1) a_{3 a+2 b}(1,1)=1$. The first half of Lemma 2 on $\{a, b\}$ finishes the proof.

It is now clear that the multiplier of $G$ has order 1 or 3 , as $\alpha_{r, s}(t, u)=1$ or $\neq 1$, for a pair $\{r, s\}$ of orthogonal roots. We shall prove that the latter case holds.

Let $B$ be the Borel subgroup $B=U H$. We shall construct a certain extension $R$ of $B$ by $C$, a cyclic group of order 3, with $C \leq Z(R) \cap R^{\prime}$, and then we shall check that the cohomology class of its cocycle in $H^{2}(B, C)$ is stable with respect to $G$ in the sense of Cartan-Eilenberg (see Chapter XII of [3]). This will then imply that $H^{2}(G, C)$ is nontrivial.

Let $R$ be the group generated by symbols $z_{r}(t), k_{a}(v), k_{b}(v), r \in \Sigma^{+}, t \in K$, $v \in K^{\times}$, which satisfy

$$
\begin{aligned}
z_{r}(t) z_{r}(u) & =z_{r}(t+u), \quad \text { all } t, u \in K, r \in \Sigma^{+}, \\
k_{r}(v) k_{r}\left(v^{\prime}\right) & =k_{r}\left(v v^{\prime}\right), \quad r=a, b ; v, v^{\prime} \in K^{\times}, \\
{\left[z_{r}(t), z_{s}(u)\right] } & =\prod_{i, j} z_{i r+j s}\left(c_{i j r s} t^{i} u^{j}\right) \beta_{r s}(t, u),
\end{aligned}
$$

where the $c_{i j r s}$, the indices, etc., are as in the formulas for $G$. We let $\beta_{r s}(t, u)=1$ 
unless $\{r, s\}$ is a pair of orthogonal roots or a pair of roots forming an angle of $150^{\circ}$. For an orthogonal pair $\{r, s\}$, we require

$$
\beta_{w_{s^{\prime}(r),} w_{s^{\prime}(s)}}\left(\eta_{s^{\prime}, r} t, \eta_{s^{\prime}, s^{\prime}} u\right)=\beta_{r, s^{\prime}}(t, u)
$$

for all $t, u \in K$ and all $w_{s^{\prime}} \in W$ such that $\left\{r, s, w_{s^{\prime}}(r), w_{s^{\prime}}(s)\right\}$ is contained in $\Sigma^{+}$. If $r$ and $s$ form an angle of $150^{\circ}$, we require $\beta_{r, s}(1,1)=\beta_{r, s}(t, u)=\beta_{w(r), w(s)}(t, u)$ for all $t, u \in K$ and all $w \in W$ such that $\left\{r, s, w_{s^{\prime}}(r), w_{s^{\prime}}(s)\right\}$ is contained in $\Sigma^{+}$.

$$
\begin{gathered}
\beta_{a, b}(1,1)=\beta_{a, 3 a+2 b}(1,1)^{-1}, \\
{\left[\beta_{r s}(t, u), z_{s^{\prime}}(v)\right]=\left[\beta_{r s}(t, u), k_{r^{\prime}}\left(v^{\prime}\right)\right]=1}
\end{gathered}
$$

for all $r, s, s^{\prime}$, for $r^{\prime}=a, b$, all $t, u, v \in K$ and $v^{\prime} \in K^{\times}$.

$$
\begin{aligned}
z_{r}(t)^{k} a^{(v)} & =z_{r}\left(t v^{c(r, a)}\right), \\
z_{r}(t)^{k_{b}(v)} & =z_{r}\left(t v^{c(r, b)}\right) \text { for all } t, v, r, \\
{\left[k_{a}(-1), k_{b}(-1)\right] } & =1 .
\end{aligned}
$$

Now, some remarks about $R$. There is a map $\rho$ of $R$ onto $B$ given by $z_{r}(t) \mapsto$ $x_{r}(t), k_{s}(v) \mapsto b_{s}(v)$. $\rho$ extends to a homomorphism since the images of the generators satisfy the relations of $R$. The group $T=\left\langle k_{r}(-1) \mid r=a, b\right\rangle$ is elementary abelian of order four and $\left.\rho\right|_{T}$ is an isomorphism onto $H . \beta_{0}=\beta_{a, 3 a+2 b}(1,1)$ has cube 1 since $\beta_{a, 3 a+2 b}(t, u)$ is central in $R$ and bilinear in $t$ and $u$. The group $S=\left\langle z_{r}(t) \mid r \in \Sigma^{+}, t \in K\right\rangle$ is therefore a normal Sylow 3-subgroup of $R$. Every element of $R$ may be written uniquely as $\Pi_{r} z_{r}\left(t_{r}\right) \cdot \beta_{0}^{i}, i=0,1,2$, where the terms in the product are ordered as the roots are in $\Sigma^{+}$. It is also clear that $R$ is the semidirect product of $S$ and $T$ because $R=\langle S, T\rangle, S T$ is a subgroup, and $S \cap T$ is a 3-subgroup of $T$, hence 1 .

By the above remarks, $|R|=|S||T|=\left|\left\langle\beta_{0}\right\rangle\right||B|$, as $\left\langle\beta_{0}\right\rangle=\operatorname{ker} \rho$. We need to establish $\beta_{0} \neq 1$. To do this, we shall construct a group isomorphic to $R$ in which the element corresponding to $\beta_{0}$ is not 1 .

First, consider the 3-group $D$ generated by elements $z_{1}, z_{2}, z_{3}, z_{4}$ subject to the relations

$$
\begin{aligned}
& z_{i}^{3}=1, \quad i=1,2,3,4, \\
& {\left[z_{1}, z_{2}\right]=z_{5}, \quad\left[z_{i}, z_{5}\right]=1, \quad i=1,2,3,4,} \\
& {\left[z_{1}, z_{4}\right]=\gamma^{-1}, \quad\left[z_{2}, z_{3}\right]=y, \quad\left[z_{i}, \gamma\right]=1, \quad i=1,2,3,4,}
\end{aligned}
$$

and all other $\left[z_{i}, z_{j}\right]=1$ if not instantly derivable from one of the above. The 
group $D$ has order $\leq 3^{6}$ because every element of $D$ can be put in the form $\Pi_{i=1}^{5} z_{i}^{e} e_{i} \gamma^{e}, e_{i}, e=1,2,3$. On the other hand $D$ must have order $3^{6}$. The correspondence $z_{1} \mapsto x_{b}(1), z_{2} \mapsto x_{3 a+b}(1), z_{3} \mapsto x_{a+b}(1), z_{4} \mapsto x_{2 a+b}(1)$ extends to a homomorphism of $D$ onto $X=\left\langle X_{b}, X_{a+b}, X_{2 a+b}, X_{3 a+b}, X_{3 a+2 b}\right\rangle$ with kernel $\langle\gamma\rangle$ such that $z_{5} \mapsto x_{3 a+2 b}(1) \neq 1$.

The correspondence $z_{i} \mapsto v_{i}, i=1,2,3,4$, extends to a homomorphism of $D$ onto an extra-special group $V$ of order $3^{5}$ with kernel $\left\langle z_{5}\right\rangle$ such that $\gamma \mapsto \delta \neq 1$. $V$ may be given by generators $v_{1}, v_{2}, v_{3}, v_{4}$ and relations $v_{i}^{3}=1,\left[v_{i}, v_{j}, v_{k}\right]=.1$, and $\left[v_{i}, v_{j}\right]=\delta^{f\left(v_{i} v_{j}\right)}$ for all $i, j, k$ where $f$ is the nonsingular symplectic form on the vector space $V / V^{\prime}$ given by the matrix

$$
\left(\begin{array}{rrrr}
0 & 0 & 0 & -1 \\
0 & 0 & 1 & 0 \\
0 & -1 & 0 & 0 \\
1 & 0 & 0 & 0
\end{array}\right)
$$

with respect to the basis $v_{i} V^{\prime}$ of $V / V^{\prime}, i=1,2,3,4$.

Next, we consider some automorphisms of $D$. Define the maps $\xi, \eta$ and $\mu$ of $D$ to $D$ by

$$
\begin{array}{rlrl}
\xi: & z_{1} & \mapsto z_{1} z_{5}^{-1} z_{2}^{-1} z_{4} z_{3} \gamma^{-1}, & z_{2} \mapsto z_{2}, \\
z_{3} \mapsto z_{3} z_{4}^{-1}, & z_{4} \mapsto z_{4} ; \\
\eta: z_{1} \mapsto z_{1}^{-1}, & z_{2} \mapsto z_{2}^{-1}, \\
z_{3} \mapsto z_{3}^{-1}, & z_{4} \mapsto z_{4}^{-1} ; \\
\mu: \quad z_{1} \mapsto z_{1}, & z_{2} \mapsto z_{2}^{-1}, \\
z_{3} & \mapsto z_{3}^{-1}, & z_{4} \mapsto z_{4} .
\end{array}
$$

That these maps extend to homomorphisms is readily checked by noting that the images of the generators $z_{i}$ under these maps satisfy the defining relations on the $z_{i}$. To see that they are isomorphisms, note that they all induce isomorphisms of $D / D^{\prime}$. Any proper kernel therefore must be contained in $D^{\prime}=\left\langle z_{s}, \gamma\right\rangle$, but trivial observations exclude this possibility. Thus, $F=\langle\xi, \eta, \mu\rangle \leq$ Aut $D$.

Direct computation shows that $\xi^{3}=1, \eta^{2}=1, \mu^{2}=1,[\eta, \xi]=[\eta, \mu]=1$, and $\mu \xi \mu=\xi^{-1}$. Hence, $F \cong\left\langle X_{a}, H\right\rangle$ via $\xi \rightarrow x_{a}(1), \eta \rightarrow b_{a}(-1), \mu \rightarrow b_{b}(-1)$.

We can now establish $\beta_{0} \neq 1$. A homomorphism of $R$ onto the semidirect product $D F$ may be defined as follows: 


$$
\begin{array}{rlrl}
z_{a}(1) & \mapsto \xi, & k_{a}(-1) & \mapsto \eta, \\
k_{b}(-1) & \mapsto \mu, \\
z_{b}(1) & \mapsto z_{1}, & z_{3 a+b}(1) & \mapsto z_{2}, \\
z_{a+b}(1) & \mapsto z_{3}, & z_{2 a+b}(1) & \mapsto z_{4}, \\
z_{3 a+2 b}(1) & \mapsto z_{5}, & \beta_{0} & \mapsto \gamma,
\end{array}
$$

We have a homomorphism because the images of the generators of $R$ satisfy the defining relations of $R$. Since we know that $\gamma \neq 1, \beta_{0} \neq 1$ follows, i.e., the extension $R$ of $B$ does not collapse.

This section is concerned $w$ ith the construction of an explicit factor set for the extension $R$ of $B$ by $C$.

Every element $x$ of $B$ may be written uniquely as $x_{1} x_{2}$, with $x_{1} \in U, x_{2} \in H$. Furthermore,

$$
\begin{aligned}
& x_{1}=x_{a}\left(t_{1}\right) x_{b}\left(t_{2}\right) x_{a+b}\left(t_{3}\right) x_{2 a+b}\left(t_{4}\right) x_{3 a+b}\left(t_{5}\right) x_{3 a+2 b}\left(t_{6}\right), \\
& x_{2}=b_{a}\left(v_{1}\right) b_{b}\left(v_{2}\right) .
\end{aligned}
$$

The $t_{i}$ and $v_{j}$ are unique elements of $K$.

Choose representatives $y=y(x)$ for $x=x_{1} x_{2} \in B$ in $R$ as follows: $y=y_{1} y_{2}$, $y_{1} \in S, y_{2} \in T$,

$$
\begin{aligned}
& y_{1}=z_{a}\left(t_{1}\right) z_{b}\left(t_{2}\right) z_{a+b}\left(t_{3}\right) z_{2 a+b}\left(t_{4}\right) z_{3 a+b}\left(t_{5}\right) z_{3 a+2 b}\left(t_{6}\right), \\
& y_{2}=k_{a}\left(v_{1}\right) k_{b}\left(v_{2}\right) .
\end{aligned}
$$

Then the factor set $b$ of this extension is well defined by $y(x) y\left(x^{\prime}\right)=y\left(x x^{\prime}\right) b\left(x, x^{\prime}\right)$.

We note that if $b \in H$, then $b(x, b)=b(b, x)=1$ for all $x \in U$. Also, $b\left(b, b^{\prime}\right)=$ 1 for any $b, b^{\prime} \in H$.

For $x, x^{\prime} \in U$, we give an expression for $b\left(x, x^{\prime}\right)$. Let $x=x_{a}\left(t_{1}\right) x_{b}\left(t_{2}\right) \cdots$ in the above notation and let $x^{\prime}=x_{a}\left(t_{1}^{\prime}\right) x_{b}\left(t_{2}^{\prime}\right) \cdots$, Then choosing $y(x), y\left(x^{\prime}\right)$ by

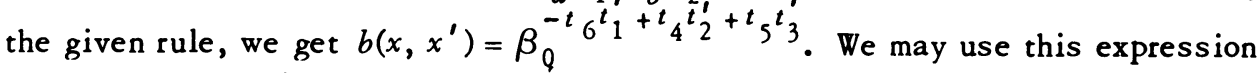
to compute $b\left(x, x^{\prime}\right)$ for any $x, x^{\prime} \in B$ as follows. Write $x=x_{1} x_{2}, x^{\prime}=x_{1}^{\prime} x_{2}^{\prime}$ with $x_{1}, x_{1}^{\prime} \in U$ and $x_{2}, x_{2}^{\prime} \in H$. By the last paragraph,

$$
\begin{aligned}
& y(x) y\left(x^{\prime}\right)=y\left(x_{1}\right) y\left(x_{2}\right) y\left(x_{1}^{\prime}\right) y\left(x_{2}^{\prime}\right) \\
& =y\left(x_{1}\right) y\left(x_{1}^{\prime}\right)^{y\left(x_{2}\right)^{-1}} y\left(x_{2}\right) y\left(x_{2}^{\prime}\right)=y\left(x_{1} x_{1}^{\prime x_{2}^{-1}}\right) b\left(x_{1}, x_{1}^{\prime x_{2}^{-1}}\right) y\left(x_{2} x_{2}^{\prime}\right) \\
& =y\left(x_{1} x_{1}^{\prime x_{2}^{-1}} x_{2} x_{2}^{\prime}\right) b\left(x_{1}, x_{1}^{x_{2}^{-1}}\right)=y\left(x x^{\prime}\right) b\left(x_{1}, x_{1}^{\prime x_{2}^{-1}}\right) .
\end{aligned}
$$


So, $b\left(x, x^{\prime}\right)=b\left(x_{1}, x_{1}^{\prime x_{2}^{-1}}\right)$, for which we can use the above formula.

The extension $R$ of $B$ by $C$ corresponds to an element of $H^{2}(B, C)$ which we also denote by $b$. We show that $b$ is stable under $G$ by showing that the restrictions of $b$ to $B \cap B^{g}$ and $B^{g^{-1}} \cap B$, for all $g \in G$, correspond under the homomorphisms of cohomology groups

$$
c_{g}: H^{2}\left(B \cap B^{g}, C\right) \rightarrow H^{2}\left(B^{g^{-1}} \cap B, C\right)
$$

which are induced by the maps

$$
\begin{aligned}
& \tilde{c}_{g}\left(a(x, y)+B^{2}\left(B \cap B^{g}, C\right)\right) \\
& \quad=a\left(x^{g^{-1}}, y^{g^{-1}}\right)+B^{2}\left(B^{g^{-1}} \cap B, C\right) \in Z^{2}\left(B^{g^{-1}} \cap B, C\right) / B^{2}\left(B^{g^{-1}} \cap B, C\right)
\end{aligned}
$$

in the usual cohomology notation (see [3] or [10]). It suffices to let $g$ range over a set of $(B, B)$ double coset representatives.

Such a set of representatives is $J=\left\{1, n^{-1}, n^{-2}, n^{-3}, n^{-4}, n^{-5}, n_{r}^{-1} \mid\right.$ $\left.r \in \Sigma^{+}\right\}$, where $n=n_{a} n_{b}$.

For $g=1, b\left(x, x^{\prime}\right)=b\left(x^{g^{-1}}\right)$, trivially.

For $g=n_{a+b}^{-1}, n_{2 a+b}^{-1}, n_{3 a+b}^{-1}, n_{3 a+2 b}^{-1}, n^{-2}, n^{-3}, n^{-4}$, the subgroups $B \cap B^{8}$ and $B^{g^{-1}} \cap B$ do not contain two elements $x, x^{\prime}$ such that $b\left(x, x^{\prime}\right) \neq 1$. Thus, $b\left(x, x^{\prime}\right)=b\left(x^{8}, x^{\prime} 8^{-1}\right)=1$ for these $g$. Hence the restrictions of $b$ represent the 0 -elements of both cohomology groups, and so correspond under the homomorphism $c_{\boldsymbol{g}}$.

For $g=n_{a}^{-1}, B \cap B^{g}=B^{g^{-1}} \cap B=\left\langle H, X_{b}, X_{a+b}, X_{2 a+b}, X_{3 a+b}, X_{3 a+2 b}\right\rangle$. In the previous notation, for $x, x^{\prime} \in U \cap U^{8} \leq B \cap B^{8}, b\left(x, x^{\prime}\right)=\beta_{0}^{t} 4^{t_{2}^{\prime}+t_{5} t_{3}^{\prime}}$. A direct computation shows that $b\left(x^{g^{-1}}, x^{g^{-1}}\right)=\beta_{0}^{-t_{4}^{\prime} t_{2}-t_{5}^{\prime} t_{3}}$. We must show that this cocycle $b^{\prime}=c_{8}\left(\left.b\right|_{B \cap B^{8}, B \cap B 8}\right)$ is cohomologous to $b^{\prime \prime}=$

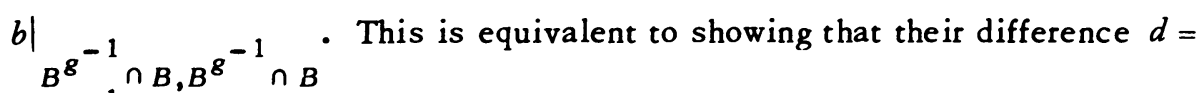
$b^{\prime \prime} b^{\prime-1}$ is the factor set of a split extension. We see that, for 3-elements $x, x^{\prime}$,

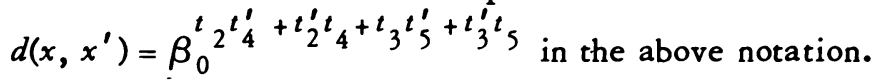

Let $1 \rightarrow C \rightarrow E \rightarrow Y \rightarrow 1$ be the restriction to $Y=U^{g^{-1}} \cap U$ of the extention of $B$ given by $d$. We claim that the extension splits.

First, note that the representatives $y(x)$ for $x \in Y^{\prime}=Z(Y)$ form a central subgroup of $E$. This is immediate from the formula for $d$. In fact, $d\left(x, x^{\prime}\right)=d\left(x^{\prime}, x\right)=$ 1 for $x \in Y^{\prime}, x^{\prime} \in Y$.

Secondly, we see that $C \underline{\nless} E^{\prime}$. We compute the commutators $\left[y(x), y\left(x^{\prime}\right)\right]$. 


$$
\begin{aligned}
y(x) y\left(x^{\prime}\right) & =d\left(x, x^{\prime}\right) y\left(x x^{\prime}\right)=d\left(x, x^{\prime}\right) y\left(x^{\prime} x\left[x, x^{\prime}\right]\right) \\
& =d\left(x, x^{\prime}\right) y\left(x^{\prime}\right) y(x) d\left(x^{\prime}, x\right)^{-1} y\left(\left[x, x^{\prime}\right]\right) .
\end{aligned}
$$

But the formula for $d$ shows $d\left(x, x^{\prime}\right)=d\left(x^{\prime}, x\right)$. So, $\left[y(x), y\left(x^{\prime}\right)\right]=y\left(\left[x, x^{\prime}\right]\right)$. Since the $y(x), x \in Y^{\prime}$, form a subgroup, we have $C \cap E^{\prime}=1$ and $C \underline{\sharp} E^{\prime}$.

Thirdly, $C E^{\prime} / E^{\prime}$ is a summand of $E / E^{\prime}$. This follows from Fitting's theorem on the abelian 3-group $E / E^{\prime}$. $E / E^{\prime}$ carries a 2-group of operators isomorphic to $H, C$ is central, and every element of $E / C E^{\prime}$ is inverted by some $y(b), b \in H$. Thus, $E / E^{\prime} \cong C \oplus E / C E^{\prime}$.

These steps imply that $E$ is a split extension. A normal complement to $C$ is the kernel of the composite $E \rightarrow E / E^{\prime} \rightarrow C E^{\prime} / E^{\prime}$.

Since $Y$ is a Sylow 3-subgroup of $B^{g^{-1}} \cap B$, and since $d$ restricted to $Y$ is cohomologous to the split extension, Gaschütz's theorem implies that $d$ is cohomologous to the split extension of $B^{g^{-1}} \cap B$.

A similar argument verifies that the restrictions of $b$ do correspond under $c_{n_{b}}$. For the remaining values $g=n^{-1}, n^{-5}$ of $J$, routine computation shows

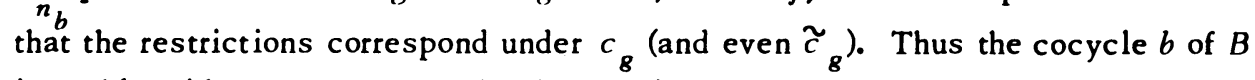
is stable with respect to $G$. The theorem is proven.

The group $F_{4}(2)$. Some notation will be given before the main theorem is stated. A root system $\Sigma$ of type $F_{4}$ may be given as the following set of vectors in four-dimensional Euclidean space with orthonormal basis $\left\{\xi_{1}, \xi_{2}, \xi_{3}, \xi_{4}\right\}$.

$$
\xi_{i} \quad \xi_{i}+\xi_{j} \quad 1 / 2\left(\xi_{i}+\xi_{j}+\xi_{k}+\xi_{l}\right)
$$

where $i, j, k, l= \pm 1, \pm 2, \pm 3, \pm 4,|i|,|j|,|k|,|l|$ are all distinct, $i^{\prime}=-i$ and $\xi_{i^{\prime}}=-\xi_{i}$ for all $i$.

Two systems of notation for these roots will be used. In the first, $\xi_{i}$ will be denoted by $i, \xi_{i}+\xi_{j}$ by $i j$, and $1 / 2\left(\xi_{i}+\xi_{j}+\xi_{k}+\xi_{l}\right)$ by $i j k l$. In the second, write the root $\xi$ as a linear combination $\Sigma_{i=1}^{4} a_{i} \xi_{i}$ and denote $\xi$ by $a_{1} a_{2} a_{3} a_{4}$ if $\xi=\xi_{i}$ or $\xi_{i}+\xi_{j}$ and by $b_{1} b_{2} b_{3} b_{4}$ if $\xi=1 / 2\left(\xi_{i}+\xi_{j}+\xi_{k}+\xi_{l}\right)$ where $b_{i}=2 a_{i}$ and where -1 is written $1^{\prime}$. The advantages of each system will become apparent.

The Dynkin diagram for $F_{4}$ is

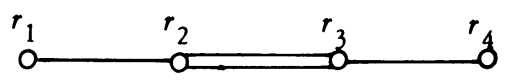

where we take our fundamental roots to be $r_{1}=11^{\prime} 1^{\prime} 1^{\prime}, r_{2}=0001, r_{3}=0011^{\prime}$, $r_{4}=011^{\prime} 0$. We order our roots by the convention $s>r$ if the first nonzero coefficient of $s-r=\Sigma_{i=1}^{4} c_{i} r_{i}$ is positive.

$F_{4}(2)$ is generated by elements $x_{r}(t), r \in \Sigma, t \in K=\mathrm{GF}(2)$. The se elements satisfy the relations: 
(A) Additivity. $x_{r}(t) x_{r}(u)=x_{r}(t+u), r \in \Sigma, t, u \in K$.

(B) Chevalley commutator relations.

$$
\left[x_{r}(t), x_{s}(u)\right]=\prod_{i, j} x_{i r+j s}\left(c_{i j r s} t^{i} u^{j}\right), \quad r \neq-s,
$$

where the product runs over all $i, j$ such that $i r+j s \in \Sigma$, the terms are arranged lexicographically, and the integers $c_{i j r s}$ depend on $\Sigma$ but not on $t$ and $u$.

The nontrivial relations $\left[x_{r}(t), x_{s}(u)\right]$ for $F_{4}(K)$ over a field $K$ of characteristic two are listed below

$$
\begin{aligned}
{\left[x_{i}(t), x_{j}(u)\right] } & =1, \quad\left[x_{i}(t), x_{i^{\prime} j}(u)\right]=x_{j}(t u) x_{i j}\left(t^{2} u\right), \\
{\left[x_{i j}(t), x_{j^{\prime} k}(u)\right] } & =x_{i k}(t u), \quad\left[x_{i}(t), x_{i^{\prime} j k l}\right]=x_{i j k l}(t u), \\
{\left[x_{i j}(t), x_{i^{\prime} j^{\prime} k l}(u)\right] } & =x_{i j k l}(t u) x_{k l}\left(t u^{2}\right), \quad\left[x_{i j k l}(t), x_{i j^{\prime} k^{\prime} l^{\prime}}(u)\right]=x_{i}(t u), \\
{\left[x_{i j k l}(t), x_{i j k^{\prime} l^{\prime}}(u)\right] } & =1 .
\end{aligned}
$$

Theorem. The Schur multiplier of $F_{4}(2)$ bas order two. The (unique) covering group may be presented with the generators $y_{t}(t), r \in \Sigma, t \in K$, and relations

$$
\begin{array}{r}
y_{r}(t) y_{r}(u)=y_{r}(t+u), \quad r \in \Sigma, \quad t, u \in K, \\
{\left[y_{r}(t), y_{s}(u)\right]=\prod_{i, j} y_{i r+j s}\left(c_{i j r s} t^{i} u^{j}\right) \cdot \alpha_{r s}(t, u),}
\end{array}
$$

where the indices $c_{i j r s}$, etc., are the same as in the formulas (B) for $F_{4}(2)$, and where $a_{r s}(t, u)=1$ unless $\{r, s\}$ consists of a short and a long root forming an angle of $135^{\circ}$ and $t, u \neq 0$. For any sucb $r, s, t, u, \alpha_{r s}(t, u)=\zeta \neq 1$ and $\langle\zeta\rangle$ is the center of the covering group.

Lemma. The Weyl group $W$ of $\Sigma$ is transitive on roots of the same length. If $\{r, s\},\left\{r^{\prime}, s^{\prime}\right\} \subseteq \Sigma, r, r^{\prime}$ (resp. $s, s^{\prime}$ ) bave the same lengths and the angle between $r$ and $s$ equals the angle between $r^{\prime}$ and $s^{\prime}$, then there is a $w \in W$ such that $w(r)=r^{\prime}$, $w(s)=s^{\prime}$.

Proof. The first part restates Lemma 5 of Chevalley [5]. So, we may assume $r=r^{\prime}$ and that $r=1000$ or 1100 as $r$ has length 1 or $\sqrt{2}$, respectively. The set $\Sigma_{0}$ of roots orthogonal to $r$ forms a root system of type $B_{3}$ or $C_{3}$, respectively, and the stabilizer $W_{0}$ of $r$ in $W$ acts as the Weyl group of $\Sigma_{0}$. Hence $W_{0}$ is transitive on roots of the same length in $\Sigma_{0}$. In general, write all $s$ of a given length and angle to $r$. It is then easy to give enough $w \in W_{0}$ to prove the transitivity of $W_{0}$ on these sets. The lemma is proven.

The lemma enables us to partition the set of pairs $\{r, s\}, r, s \in \Sigma$ into families of $W$-conjugate pairs denoted by triples $(,$, ). The first two (unordered) entries 
are $(l, l),(s, s)$ or $(l, s)$ as $r$ and $s$ are both long, both short, or of different lengths, and the last entry is the angle between $r$ and $s$. We list these families:

$$
\begin{array}{lll}
\left(l, l, 0^{\circ}\right) & \left(s, s, 0^{\circ}\right) & \left(l, s, 90^{\circ}\right) \\
\left(l, l, 180^{\circ}\right) & \left(s, s, 180^{\circ}\right) & \left(l, s, 45^{\circ}\right) \\
\left(l, l, 9^{\circ}\right) & \left(s, s, 90^{\circ}\right) & \left(l, s, 135^{\circ}\right) \\
\left(l, l, 60^{\circ}\right) & \left(s, s, 60^{\circ}\right) & \\
\left(l, l, 120^{\circ}\right) & \left(s, s, 120^{\circ}\right) &
\end{array}
$$

Denote by $U$ the subgroup of $F_{4}(2)=G$ generated by all $x_{r}(1)=x_{r}$, for $r \in \Sigma^{+}$. Then $U$ is a Sylow 2-subgroup of $G$.

Lemma. The commutator subgroup $U^{\prime}$ of $U$ contains $x_{r}, r \in \Sigma^{+}, r \neq 0001$, $0010,0100,11^{\prime} 11^{\prime} 1$, $011^{\prime} 0,0011,11^{\prime} 00,0011^{\prime}$.

Proof. By direct calualation.

Lemma. If $\{r, s\} \in\left(l, l, 60^{\circ}\right), .\left(s, s, 60^{\circ}\right),\left(l, s, 45^{\circ}\right)$, then there is a $w \in W$ with $w(r)=1000$ if $r$ is short, $w(r)=1100$ if $r$ is long, and $x_{w(s)} \in U^{\prime}$.

Proof. Use the two previous lemmas.

Let $\hat{G}$ be a covering group of $G$. Letting $A=Z(\hat{G})$, we have $A \subseteq \hat{G}^{\prime}$ and $\hat{G} / A \cong G$. We may think of $G$ as the quotient group $\hat{G} / A$, the elements of $G$ as cosets, etc., when convenient. Let $V$ be the preimage of $U$ in $G$; then $V / A=U$.

Select $\tilde{y}_{r}(t) \in x_{r}(t)$ for all $r \in \Sigma, t \in K$, and define

$$
\begin{aligned}
y_{i k}(t u) & =\left[\tilde{y}_{i j}(t), \tilde{y}_{j^{\prime} k}(u)\right], \\
y_{i}(t u) & =\left[\tilde{y}_{i j k l}(t), \tilde{y}_{i j^{\prime} k^{\prime} l^{\prime}}(u)\right], \\
y_{i j k l}(t u) & =\left[\tilde{y}_{i}(t), \tilde{y}_{i^{\prime} j k l}(u)\right] .
\end{aligned}
$$

These elements are well defined because the commutators depend only on the coset of each argument modulo $A$. They enjoy the property $y_{r}(t)^{\hat{n}_{w}}=y_{w(r)}(t)$ for all $w \in W$, where $\hat{n}_{w} \in n_{w}$. To see this, write $r=s+s^{\prime}$ with $r, s, s^{\prime}$ of the same length. Then

$$
y_{r}(t)^{\hat{n}} w=\left[y_{s}(t), y_{s^{\prime}}(1)\right]^{\hat{n}} w=\left[y_{w(s)}(t), y_{w\left(s^{\prime}\right)}(1)\right]=y_{w(r)}(t)
$$

The relations (A) and (B) hold for the $y_{r}(t)$ modulo a factor from $A$ :

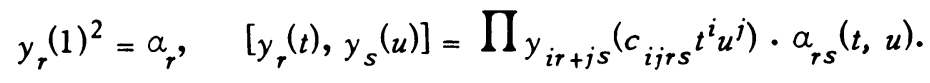

We shall determine the $a_{r}$ and $a_{r s}(t, u)$. Abbreviate $y_{r}=y_{r}(1)$. 
Lemma. $a_{r, s}(t, u)=1$ for $\{r, s\} \notin\left(l, s, 135^{\circ}\right)$.

Proof. If $r, s$ are orthogonal, let $\Sigma_{0}$ be the roots orthogonal to $r$. Then, $X_{0}=$ $\left\langle x_{r} \mid r \in \Sigma_{0}\right\rangle \cong \mathrm{Sp}(6,2)$, simple. So, $\left[\left\langle y_{r}\right\rangle, \hat{X}_{0}\right]=1$ as $\left[\left\langle x_{r}\right\rangle, X_{0}\right]=1$. If $r, s$ form a $60^{\circ}$ or $45^{\circ}$ angle, there is a $w \in W$ with $w(r)=1000$ or 1100 and $x_{w(s)} \in U^{\prime}$. Since $x_{w(r)} \in Z(U), y_{w(r)} \in Z_{2}(V)$, the second center of $V$. So,

$$
\left[y_{w(r)}, y_{w(s)}\right] \in\left[Z_{2}(V), A V^{\prime}\right]=1
$$

giving $a_{r, s}(t, u)=1$ in this case. Finally, $a_{r, s}(t, u)=1$ for $\{r, s\} \in\left(l, l, 120^{\circ}\right)$, $\left(s, s, 120^{\circ}\right)$ by definition of the $y_{r}(t)$.

Corollary. $y_{r}^{2}=1$ for all $r \in \Sigma$, i.e., $\alpha_{r}=1$.

Proof. Write $r=s+s^{\prime}$ where $r, s, s^{\prime}$ have the same length and $r$ forms an angle of $60^{\circ}$ with $s$ and $s^{\prime}$. So,

$$
\begin{aligned}
1 & =\left[y_{s^{\prime}}^{2}, y_{s^{\prime}}\right]=\left[y_{s}, y_{s^{\prime}}\right]^{y} s\left[y_{s}, y_{s^{\prime}}\right] \\
& =\left[y_{s^{\prime}}, y_{s^{\prime}} y_{s+s^{\prime}}\right]\left[y_{s}, y_{s^{\prime}}\right]=\left[y_{s^{\prime}} y_{s^{\prime}}\right]\left[y_{s^{\prime}}, y_{s^{\prime}}\right]=y_{r}^{2},
\end{aligned}
$$

by the lemma.

Lemma. For $\{r, s\},\left\{r^{\prime}, s^{\prime}\right\} \in\left(l, s, 135^{\circ}\right), \alpha_{r s}(t, u)^{2}=1$ and $\alpha_{r s}(t, u)=\alpha=$ $a_{r^{\prime}, s^{\prime}}(t, u)$, for $t, u \neq 0$.

Proof. Using the last lemma, expand the commutator $\left[x_{r}^{2}, x_{s}\right]=1$ for the first assertion. The second part follows from $y_{r}(t)^{n} w=y_{w(r)}(t)$.

By the theorems of R. Steinberg [15], the abstract group generated by symbols $x_{r}(t), r \in \Sigma, t \in K$, satisfying relations (A) and (B), is isomorphic to $F_{4}(2)$. Our results so far show that the Schur multiplier of $F_{4}(2)$ has order 1 or 2 , as $\alpha=1$ or $\alpha \neq 1$ respectively. We must show $\alpha \neq 1$.

Let $r_{0}=1100$ be the root of maximal height in the ordering we have given for $\Sigma$. Let $\Sigma_{0}$ consist of the roots orthogonal to $r_{0}, \Sigma_{0}^{+}=\Sigma^{+} \cap \Sigma_{0}, \Sigma_{1}=\Sigma^{+} \backslash \Sigma_{0}$. Then $P=\left\langle x_{r} \mid r \in \Sigma^{+} \cup \Sigma_{0}\right\rangle=C_{G}\left(x_{r_{0}}\right)$ is a parabolic subgroup, $O_{2}(P)=\left\langle x_{r} \mid r \in \Sigma_{1}\right\rangle$, and $S=\left\langle x_{r} \mid r \in \Sigma_{0}\right\rangle=C_{3}(2)=\mathrm{Sp}(6,2) . P=O_{2}(P) \cdot S$, a semidirect product. $\left|\Sigma_{0}\right|=18,\left|\Sigma_{1}\right|=15$, and

$$
\Sigma_{0}^{+}=\left\{11^{\prime} 00,0011,0011^{\prime}, 0001,0010,11^{\prime} 11,11^{\prime} 1^{\prime} 1,11^{\prime} 11^{\prime}, 11^{\prime} 1^{\prime} 1^{\prime}\right\}
$$

We shall construct a nonsplit extension $R$ of $U$ by $C \cong Z_{2}$ and show that the corresponding element of $H^{2}(U, C)$ is stable with respect to $G=F_{4}(2)$ in the sense of Cartan-Eilenberg [3]. This will imply $H^{2}(G, C) \neq 1$ and hence that $\alpha \neq 1$. 
Let $X=O_{2}(P)$ and set $T=\left\langle x_{r} \mid r \in \Sigma_{0}^{+}\right\rangle$. We wish to define an action of $T$ on $Y=X \times C$. To do this, we will define certain automorphisms $z_{s}, s \in \Sigma_{0}^{+}$, of $Y$ and show that $T$ is isomorphic to the subgroup $Q=\left\langle z_{s} \mid s \in \Sigma_{0}\right\rangle \subseteq$ Aut $Y$.

The group $Y=X \times C=\left\{\left(x, \gamma^{i}\right) \mid x \in X, i=0,1, C=\langle\gamma\rangle\right\}$ may be presented as the group generated by $\left(x_{r}, 1\right), r \in \Sigma_{1},(1, \gamma)$ satisfying the appropriate relations among those of (A) and (B), plus $(1, \gamma)^{2}=1,\left[\left(x_{r}, 1\right),(1, \gamma)\right]=1$, all $r \in \Sigma_{1}$. Now, for each $s \in \Sigma_{0}^{+}$, define $z_{s}: Y \rightarrow Y$ by $(1, \gamma)^{z_{s}}=(1, \gamma),\left(x_{r}, 1\right)^{z_{s}}=\left(x_{r}\left[x_{r}, x_{s}\right]\right.$, $\left.\gamma^{e(r, s)}\right)$, where $e(r, s)=1$ if $r, s \in\left(l, s, 135^{\circ}\right), e(r, s)=0$ otherwise. The $z_{s}$ extend to automorphisms because the images of the generators of $Y$ under $z_{s}$ satisfy the relations defining $Y$. For later use, we may write $z_{s}(t)=\left\{\begin{array}{ll}1, & t=0 \\ z_{s}, & t=1\end{array}\right.$.

If $W_{0}$ is the stabilizer of $r_{0}$ in $W$, then $W_{0}$ acts as the Weyl group of $\Sigma_{0}$ on $\Sigma_{0}$. Arguing as before, $W_{0}$ is transitive on pairs $\{r, s\}, r, s \in \Sigma_{0}, r \neq \pm s$ with the same number of long and short roots which make a fixed angle between them. These families (listed below in previous notation) meet seven of the nine families of $\Sigma$. Since the definition of the action of $z_{s}$ on the $\left(x_{r}, 1\right)$ is invariant under application of $w \in W_{0}$ to the indices (provided $s, w(s) \in \Sigma_{0}^{+}$) it is sufficient to compute $\left[z_{s}\right.$, $\left.z_{s^{\prime}}\right]$ on each $\left(x_{r}, 1\right)$ for a representative $\left\{s, s^{\prime}\right\}$ from each of the seven relevant families.

A set of representatives $s, s^{\prime}$ for these families is as follows:

$$
\begin{array}{ll}
\left\{11^{\prime} 00,0011^{\prime}\right\} \in\left(l, l, 90^{\circ}\right) & \{0010,0001\} \in\left(s, s, 90^{\circ}\right), \\
\phi=\Sigma_{0}^{+} \cap\left(l, l, 60^{\circ}\right), & \left\{0010,11^{\prime} 11\right\} \in\left(s, s, 60^{\circ}\right), \\
\phi=\Sigma_{0}^{+} \cap\left(l, l, 120^{\circ}\right) & \left\{0010,11^{\prime} 1^{\prime} 1\right\} \in\left(s, s, 120^{\circ}\right),
\end{array}
$$

$$
\begin{aligned}
& \left\{11^{\prime} 00,0010\right\} \in\left(l, s, 90^{\circ}\right), \\
& \left\{0011^{\prime}, 0010\right\} \in\left(l, s, 45^{\circ}\right), \\
& \left\{0011^{\prime}, 0001\right\} \in\left(l, s, 135^{\circ}\right) .
\end{aligned}
$$

Since the $z_{s}$ leave $C=\langle(1, \gamma)\rangle$ invariant, we see that the group of automorphisms induced by $Q=\left\langle z_{s} \mid s \in \Sigma_{0}^{+}\right\rangle$on $Y / C \cong X$ is isomorphic to $T$. We must show that this homomorphism of $Q$ onto $T$ is faithful. $T$ may be presented as the abstract group generated by the $x_{r}, r \in \Sigma_{0}^{+}$, subject to the appropriate relations (A) and (B). It is therefore sufficient to show that these same relations hold among the $z_{s}$.

The relations (A), namely $z_{s}^{2}=1$, are easily checked.

For each of the seven pairs, the relations (B) may be checked from the table below, which gives $\left[\left(x_{r}, 1\right), z_{s}\right]$ in the row $r$, column $s$. The generators $\left(x_{r_{0}}, 1\right)$ and $(1, \gamma)$ are omitted since they are central in $R$. This establishes $Q \cong T$.

The semidirect product $R=Y Q$ is our desired extension of $U$ by $C . R$ does not split over $C$ as $C \subseteq Z(R) \cap R^{\prime}$.

We now define an explicit factor set for this extension. In addition to our 
given ordering $<$ on $\Sigma$, we use another ordering $\prec$ on the roots of $\Sigma^{+}$, defined by $r \prec s$ whenever $r$ is short, $s$ is long, or else $r<s$ when $r$ and $s$ have equal lengths. By an easy argument, every element $x \in U$ can be written uniquely as $\Pi_{r} x_{r}\left(t_{r}\right)$, where $r$ runs over $\Sigma^{+}$in the order determined by $\prec$, and $t_{r} \in K$. A coset representative $y(x)$ for $x \in U \cong R / C$ in $R$ is chosen as follows: $y(1)=1, y(x)=\Pi_{r} y\left(x_{r}\left(t_{r}\right)\right.$ ), where for $s$ short

$$
y\left(x_{s}\right)= \begin{cases}\left(x_{s}, 1\right), & s \in \Sigma_{1} \\ z_{s}, & s \in \Sigma_{0}^{+}\end{cases}
$$

and for $r$ long

$$
y\left(x_{r}\right)= \begin{cases}\left(x_{r}, 1\right), & r \in \Sigma_{1}, \\ z_{r} \cdot(1, y) & r \in \Sigma_{0}^{+}\end{cases}
$$

The factor set $b\left(x, x^{\prime}\right)$ of this extension is therefore well defined by $y(x) y\left(x^{\prime}\right)=$ $y\left(x x^{\prime}\right) b\left(x, x^{\prime}\right)$.

The extension $R$ of $U$ by $C$ corresponds to an element of $H^{2}(U, C)$ which we also denote by $b$. We show that $b$ is stable under $G$ by showing that the restrictions of $b$ to $U \cap U^{g}$ and $U^{g-1} \cap U$, for all $g \in G$, correspond under the homomorphisms of cohomology groups $c_{g}: H^{2}\left(U \cap U^{g}, C\right) \rightarrow H^{2}\left(U^{g^{-1}} \cap U, C\right)$ which are induced by the maps of cocycles $\tilde{c}_{g}(a(x, y))=a\left(x^{g^{-1}}, y^{g^{-1}}\right) \in Z^{2}\left(U^{g^{-1}} \cap U, C\right)$, for $a(x, y) \in Z^{2}\left(U \cap U^{g}, C\right)$, in the usual cohomology notation (see [3] or [10]). It suffices to let $g$ range over a set of $(U, U)$-double coset representatives. The subgroup $N$ forms such a set of representatives $\left(H=1\right.$ in $F_{4}(2)$, so $\left.N \cong W\right)$. Every element of $N$ is of the form $n_{w}, w \in W$.

By an induction argument, we shall establish stability by proving the stronger result

$$
\begin{cases}{ }_{g}^{c}\left(\left.b\right|_{U \cap U^{g}}\right)=\left.b\right|_{U^{g^{-1}} \cap U} & \text { for all } g \in N, \\ \text { i.e., } b\left(x, x^{\prime}\right)=b\left(x^{g^{-1}}, x^{\prime g^{-1}}\right) & \text { for all } g \in N ; x, x^{\prime} \in U \cap U^{g} .\end{cases}
$$

Given $g$, we call a pair $\left(x, x^{\prime}\right)$ for which $(\dagger)$ holds a $g$-stable pair.

Define $\operatorname{supp}(x)=\left\{r \mid t_{r} \neq 0\right\}$ for $x=\Pi_{r} x_{r}\left(t_{r}\right)$. Note that $b\left(x, x^{\prime}\right)=1$ if $\operatorname{supp}(x)$ consists entirely of short roots or if $\operatorname{supp}\left(x^{\prime}\right)$ consists entirely of long roots. Since these conditions are $g$-invariant, $\left(x, x^{\prime}\right)$ is a pair stable under all $g \in N$ with $x, x^{\prime} \in U \cap U^{g}$.

$$
\text { If } x=x_{r_{1}} \cdots x_{r_{k}}, x=\xi \eta, \xi=x_{r_{1}} \cdots x_{r_{i}}, \eta=x_{r_{i_{+}}} \cdots x_{r_{k}} \text {, then } x \in U \cap U^{g}
$$
implies $\xi, \eta \in U \cap U^{g}$, and conversely. 


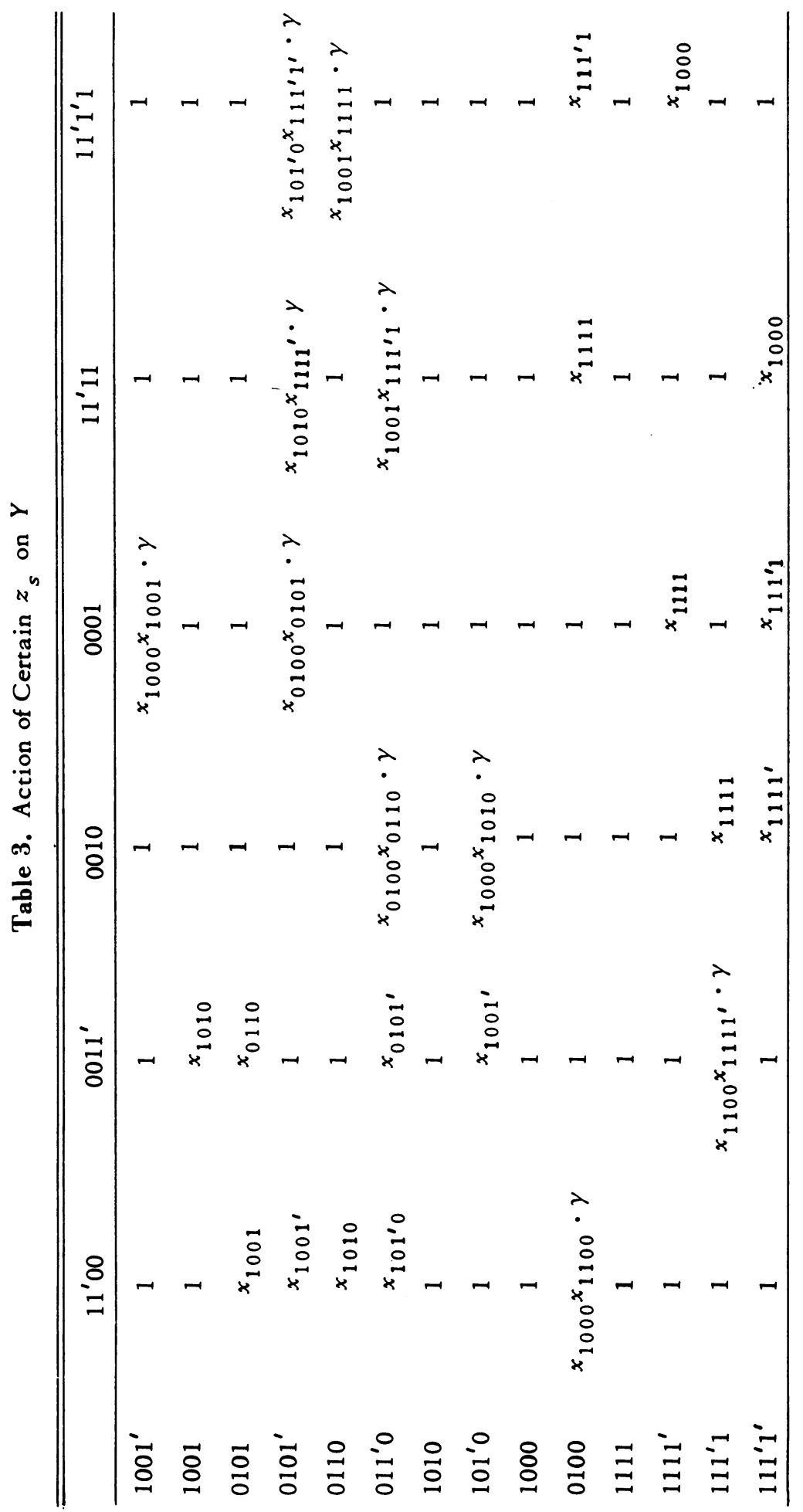


If $|\operatorname{supp}(x)|>1$, then write $x=\xi \eta$ as above. Using

$$
\begin{aligned}
b\left(x, x^{\prime}\right) & =b\left(\xi \eta, x^{\prime}\right)=y\left(\xi \eta x^{\prime}\right)^{-1} y(\xi \eta) y\left(x^{\prime}\right) \\
& =y\left(\eta x^{\prime}\right)^{-1} y(\xi)^{-1} b\left(\xi, \eta x^{\prime}\right) y(\xi) y(\eta) b(\xi, \eta)^{-1} y\left(x^{\prime}\right) \\
& =b\left(\eta, x^{\prime}\right) b\left(\xi, \eta x^{\prime}\right) b(\xi, \eta)^{-1}
\end{aligned}
$$

we obtain $b\left(x, x^{\prime}\right)=b\left(x^{g^{-1}}, x^{\prime g^{-1}}\right)$ for $g \in N$ such that $x, x^{\prime} \in U \cap U^{g}$ by induction on $|\operatorname{supp}(x)|$. We are thus reduced to proving $(\dagger)$ for $x$ such that $|\operatorname{supp}(x)|=1$, i.e., $x=x_{r}, r \in \Sigma^{+}$.

Our first case is $x=x_{r}, x^{\prime}=x_{s}, r, s \in \Sigma^{+}$. If $r$ is short, or $s$ is long, then $\left(x_{r}, x_{s}\right)$ is a $g$-stable pair, for the relevant $g \in N$, by previous remarks. So, we assume $r$ is long, $s$ short. By

$$
\begin{aligned}
b\left(x_{r}, x_{s}\right) & =y\left(x_{r} x_{s}\right)^{-1} y\left(x_{r}\right) y\left(x_{s}\right)=y\left(x_{s} x_{r}\left[x_{r}, x_{s}\right]\right)^{-1} y\left(x_{r}\right) y\left(x_{s}\right) \\
& =y\left(\left[x_{r}, x_{s}\right]\right)^{-1} y\left(x_{r}\right)^{-1} y\left(x_{s}\right)^{-1} y\left(x_{r}\right) y\left(x_{s}\right) \cdot b\left(x_{r},\left[x_{r}, x_{s}\right]\right),
\end{aligned}
$$

if $r$ and $s$ form an angle not $135^{\circ}$, then $x_{r}, x_{s}$ commute and so do $y\left(x_{r}\right)$ and $y\left(x_{s}\right)$ which implies $b\left(x_{r}, x_{s}\right)=1$. If $r$ and $s$ form an angle of $135^{\circ}$, then $\left[x_{r}, x_{s}\right]=$ $x_{r+s} x_{r+2 s}$. For all such pairs $r, s,\left[y\left(x_{r}\right), y\left(x_{s}\right)\right]=y\left(x_{r+s}\right) y\left(x_{r+2 s}\right) \cdot(1, \gamma)=$ $y\left(x_{r+s} x_{r+2 s}\right) \cdot(1, y)$ by our rules for choosing $y(x)$ and the fact that $r+s$ is short, $r+2 s$ is long. Now $b\left(x_{r},\left[x_{r}, x_{s}\right]\right)=b\left(x_{r}, x_{r+s}\right) b\left(x_{r} x_{r+s}, x_{r+2 s}\right) b\left(x_{r+s}, x_{r+2 s}\right)^{-1}$. The last two terms are 1 since $r+2 s$ is long. The first term $b\left(x_{r}, x_{r+s}\right)=1$ since $\left[x_{r}, x_{r+s}\right]=1$ by a previous case. So, for such $\{r, s\}, b\left(x_{r}, x_{s}\right)=(1, \gamma)$. In all cases, therefore, $\left(x_{r}, x_{s}\right)$ is a stable pair.

We introduce the order relation $\ll$ on $U$. We say that $x_{1} \ll x_{2}$ if

(i) the least $(\prec)$ member of $\operatorname{supp}\left(x_{1}\right)$ is $>$ that of $\operatorname{supp}\left(x_{2}\right)$,

(ii) when these are the same, $\left|\operatorname{supp}\left(x_{1}\right)\right|<\left|\operatorname{supp}\left(x_{2}\right)\right|$,

(iii) when both these are the same, the least $(\prec)$ root in

$$
\left(\operatorname{supp}\left(x_{1}\right) \cup \operatorname{supp}\left(x_{2}\right)\right) \backslash\left(\operatorname{supp}\left(x_{1}\right) \cap \operatorname{supp}\left(x_{2}\right)\right) \text { belongs to } \operatorname{supp}\left(x_{2}\right) \text {. }
$$

In general, if $x^{\prime}=\mu \nu$,

$$
\begin{aligned}
b\left(x, x^{\prime}\right) & =b(x, \mu \nu)=y(x \mu \nu)^{-1} y(x) y(\mu \nu) \\
& =y(\nu)^{-1} y(x \mu)^{-1} b(x \mu, \nu) y(x) y(\mu) y(\nu) b(\mu, \nu)^{-1} \\
& =y(\nu)^{-1} b(x, \mu) b(x \mu, \nu) y(\nu) b(\mu, \nu)^{-1}=b(x, \mu) b(x \mu, \nu) b(\mu, \nu)^{-1} .
\end{aligned}
$$

We shall prove $(\dagger)$ by $<$-induction on the second argument $x^{\prime} .(\dagger)$ holds if $\left|\operatorname{supp}\left(x^{\prime}\right)\right|=0$, or 1 as shown before. In our special case of $(* *)$ set $x=x_{r}, x^{\prime}=$ $x_{s_{1}} \cdots x_{s_{t}}, t>1, \mu=x_{s_{1}}, \nu=x_{s_{2}} \cdots x_{s_{t}}$. By previous remarks we may assume $r$ is long, $s=s_{1}$ is short. On the right side of $(* *)(\mu, \nu)$ is stable by <--induction, 
$(x, \mu)$ is stable as $|\operatorname{supp}(\mu)|=1 . b(x \mu, \nu)$ is the remaining term. If $r, s$ form an angle of $45^{\circ}$ or $90^{\circ}$ then $x \mu=\mu x$. Now, (*) implies $b(x \mu, \nu)=b(\mu, x \nu) b(\mu, x)$ is stable as $(x, \nu)$ is by $<<-$-induction and the others are, since $\operatorname{supp}(\mu)=\{s\}, s$ short. Finally if $r$ and $s$ form an angle of $135^{\circ}$, then $x \mu=\mu x x_{r+s} x_{r+2 s}=\left(\mu x_{r+s}\right) \cdot$ $\left(x x_{r+2 s}\right)$, grouping by root lengths. By $(*)$,

$$
b(x \mu, \nu)=b\left(\mu x_{r+s} x x_{r+2 s}, \nu\right)=b\left(x x_{r+2 s}, \nu\right) b\left(\mu x_{r+s}, x x_{r+2 s} \nu\right) b\left(\mu x_{r+s}, x x_{r+2 s}\right) .
$$

The last two terms are stable as $\operatorname{supp}\left(\mu x_{r+s}\right)$ consists of short roots. Again (*) implies $b\left(x x_{r+2 s}, \nu\right)=b\left(x_{r+2 s}, \nu\right) b\left(x, x_{r+2 s} \nu\right) b\left(x, x_{r+2 s}\right)$. Since $r+2 s$ is long, the last term is stable, and the first two terms are stable by $<<-$-induction (n.b. the $\prec-$ minimal member of $\operatorname{supp}\left(x_{r+2 s} \nu\right)$ is $s_{2}$, not $\left.r+2 s\right)$. Putting all this together, we have expressed $b\left(x, x^{\prime}\right)$ as a product of stable terms, so $\left(x, x^{\prime}\right)$ is a stable pair.

Our claim $(\dagger)$ is established. The proof of the theorem is complete.

\section{CHAPTER II. THE STEINBERG VARIATIONS}

Let $G_{0}$ be a Chevalley group and $G$ the Steinberg variation (if $G_{0}$ admits one) in which the field centralized by the associated field automorphism has $q=$ $p^{f}$ elements, $p$ a prime.

Steinberg proved in [16], that if $G$ has type ${ }^{2} A_{n}$ (odd $\left.n>2\right),{ }^{2} D_{n}(n \geq 4)$, ${ }^{3} D_{4}$, or ${ }^{2} E_{6}$, then the linear representations of the group $\Gamma$ presented by the relations (A) and (B) (stated below for each type) cover projective representations for $G$ over an algebraically closed field of characteristic $p$. Thus, if $\hat{G}$ is the covering group for $G$, then there is a homomorphism $\phi$ of $\hat{G}$ onto $\Gamma, A=\operatorname{ker} \phi \subseteq$ $Z(G) \cap G^{\prime}$, and $A$ is a finite p-group. $A$ is the Schur multiplier of $\Gamma$ and also the $p$-primary part of the multiplier of $G$. The corresponding statements for ${ }^{2} A_{n}(q)$, $n$ even, were not established in [16]. However, Steinberg has recently proven these statements for ${ }^{2} A_{n}(q), n \geq 4, n$ even. The case $n=2$ is handled in this paper.

Our task, then, is to determine the multiplier of $\Gamma$. In the case of ${ }^{2} A_{n}(q), n$ even, we can determine the $p$-part of $M(G)$ (which turns out to be trivial) merely by showing that in any central extension $\tilde{G}$ of $G$ by $A \cong Z_{p}$, the induced extension of $U$ splits, where $U$ is a unipotent (Sylow $p-$ ) subgroup of $G$.

Preparation for the proof. The reader is expected to know the standard machinery for Chevalley groups, [4], [5], [17], especially the root systems, which are detailed in [2].

By abusing terminology slightly, we write $U$ for the $U^{1}$ (or $U^{2}$ ) of [17]-that is, the fixed points in the standard unipotent subgroup of the Chevalley group under the outer automorphism defining $G$. Similarly, we write $H$ for $H^{1}, N$ for $N^{1}, W$ for $W^{1}$, etc. We write $t \mapsto \bar{t}$ for the associated field automorphism, $r \mapsto \bar{r}$ for the associated permutation of roots. For the trially twisted groups, these maps have 
order 3 and are written $t \mapsto \overline{\bar{t}}, r \mapsto \bar{r} \mapsto \overline{\bar{r}}$.

If $G_{0}$ has root system $\Sigma$, the "twisted" root system for $G$ is denoted ${ }^{2} \Sigma$ (or ${ }^{3} \Sigma$ if $\left.G={ }^{3} D_{4}(q)\right)$. See [17] for details and notation. If $R, S$ are roots of ${ }^{2} \Sigma$ or ${ }^{3} \Sigma$ ), we say they are long, short, orthogonal, etc. as elements of the "twisted" root system. If $G \neq{ }^{3} D_{4}(q)$ or ${ }^{2} A_{n}(q), n$ even, a long (short) root of ${ }^{2} \Sigma$ consists of one (two) roots of $\Sigma$. If $G={ }^{2} A_{n}(q), n$ even, a long root consists of three roots, and a short root of two roots. If $G={ }^{3} D_{4}(q)$ a long root consists of one root, a short root of three roots.

$K_{0}$ denotes the ground field of $q$ elements and $K$ the extension field of $q^{2}$ (or $q^{3}$ ) elements.

Suppose $G \neq{ }^{3} D_{4}(q),{ }^{2} A_{n}(q), n$ even. If $R=\{r\}, S=\{s, \bar{s}\} \in{ }^{2} \Sigma$, the corresponding "one parameter elements" are $x_{r}(t), t \in K_{0}$, and $x_{s}(u) x_{\bar{s}}(\bar{u}), u \in K$. We define $x_{R}(t)=x_{r}(t)$. However, for $S$, the one parameter element could be denoted $x_{S}(u)$ or $x_{S}(\bar{u})$. One could conceivably make a choice for a fixed $S$, then extend to other short roots under conjugation by certain elements of $N$. There are problems with the process, as the section on ${ }^{2} E_{6}(2)$ illustrates. But this is unnecessary for most of our purposes. That is, within a calculation involving various $x_{S}(u), S$ short, we fix an arbitrary choice for each $S$ which may be inferred from the context. A similar situation holds for short roots in ${ }^{3} D_{4}(q)$, and for short and long roots in ${ }^{2} A_{n}(q), n$ even.

If $R$ is the latter kind of root, $x_{r}(t) x_{\bar{r}}(\bar{t}) x_{r+\bar{r}}(u)=x_{\bar{r}}(\bar{t}) x_{r}(t) x_{r+\bar{r}}\left(u-N_{r \bar{r}} t \bar{t}\right)$ is denoted $x_{R}(t, u)$ or $x_{R}\left(\bar{t}, u-N_{r r}-t \bar{t}\right)$. (The $N_{r s}$ are certain constants related to the root system.)

As in $[17, \S 2]$, we may think of ${ }^{2} \Sigma$ as a subset of the lattice in Euclidean space generated by $\Sigma$ and $W$ as a subgroup of the Weyl group of $\Sigma$. The reflection $w_{R}$ corresponding to $R$ is $w_{r}$ if $R=\{r\}, w_{r} w_{\bar{r}}$ if $R=\{r, \bar{r}\}, w_{r} w_{r} w_{\bar{r}}$ if $R=\{r, \bar{r}$, $\overline{\bar{r}}\}$, and $w_{r+\bar{r}}$ if $R=\{r, \bar{r}, r+\bar{r}\}$. The corresponding element of $N$ is $n_{R}=n_{r}, n_{r} n_{\bar{r}}$, $n_{r} n_{r} n_{\bar{r}}, n_{r+\bar{r}}$ respectively $\left(n_{r}=x_{r}(-1) x_{-r}(1) x_{r}(-1)\right)$. The elements of $H$ correspond to self-conjugate characters on the lattice determined by $\Sigma$. We write $b_{R}(\lambda)$ for $b_{r}(\lambda), b_{r}(\lambda) b_{\bar{r}}(\bar{\lambda}), b_{r}(\lambda) b_{\bar{r}}(\bar{\lambda}) b_{\bar{r}}(\overline{\bar{\lambda}})$ respectively, where

$$
b_{r}(\lambda)=x_{r}\left(\lambda^{-1}\right) x_{-r}(-\lambda) x_{r}\left(\lambda^{-1}\right) n_{r}^{-1} \text {. }
$$

The ambiguity here for $|R| \neq 1$ is handled as for one-parameter elements.

A word of caution. $n_{R}$ permutes the $X_{S}$ as $w_{R}$ permutes the roots $S$. However, $x_{S}(t)$ may be conjugated under $n_{R}$ to $x_{S^{\prime}}( \pm t)$ or $x_{S^{\prime}}( \pm \bar{t})$, depending on our definition of the one-parameter elements for $|S| \neq 1$.

Unless $R$ is long in ${ }^{2} A_{n}(q), n$ even, the additivity relations of type (A) read $x_{R}(t) x_{R}(u)=x_{R}(t+u)$. The relations of type (B), the analogues of the Chevalley commutator relations, are stated as we treat each family. For each family, we choose representatives $y_{R}(t)$ for $x_{R}(t)$ in $\hat{G}$. The $y_{R}(t)$ satisfy (A) and (B) up to 
a factor (usually written $d$ or $f$ ) from $A$. If $G \neq{ }^{2} A_{n}(q), n$ even, we can get $\hat{G} \cong \Gamma$ (equivalently, $m(\Gamma)=1$ ) if we show that the $y_{R}(t)$ satisfy the relations $(A)$ and (B). The exceptions are handled individually, and the slightly different argument for ${ }^{2} A_{n}(q), n$ even, is discussed later.

For convenience, we identify $\Gamma$ with $\hat{G} / A$ and regard elements of $\Gamma$ as cosets of $A$ in $G . \tilde{x} \in \hat{G}$ denotes an arbitrary element of $x \in \Gamma$. Commutators in $G$ and conjugating elements depend only on their $A$-coset. Hence, elements and subgroups of $\Gamma$ operate on $\hat{G}$.

We say that a system of representatives $y_{1}, y_{2} \cdots$ in $G$ for $x_{1}, x_{2} \cdots$ in $\Gamma$ has Property (C) if $y_{i}^{n}=y_{j}, n \in N$, whenever $x_{i}^{n}=x_{j}$; and Property (I) if $y_{i} y_{j}=y_{k}$ whenever $x_{i} x_{j}=x_{k}$, and $x_{i}, x_{j}, x_{k}$ belong to the same one-parameter subgroup.

Despite the warning three paragraphs ago, to show that the factor $f_{R, s}(t, u) \in A$ in a relation $\left[y_{R}(t), y_{S}(u)\right]=\cdots$ of type (B) is identically 1 , it is enough to prove triviality for $f_{R^{\prime}, S^{\prime}}(t, u)$, when there is a $w \in W$ with $w(R)=R^{\prime}, w(S)=S^{\prime}$, once we know the $y_{R}(t), y_{S}(u), y_{R^{\prime}}\left(t^{\prime}\right), y_{S^{\prime}}\left(u^{\prime}\right)$ involved satisfy (C).

Lemma 1. Suppose that a long root $R$ in ${ }^{2} \Sigma$ is written $R=S+S^{\prime}=T+T^{\prime}$ where $S, S^{\prime}, T, T^{\prime}$ are short. Then there is a $w \in W$, with $w(S)=T, w\left(S^{\prime}\right)=T^{\prime}$.

Proof. Since $W$ is transitive on roots of the same length (2.8 of [5]), we may assume $R=\{r\}$, where $r$ is a root of maximal height in $\Sigma$. Let $S=\{s, \bar{s}\}, S^{\prime}=$ $\left\{s^{\prime}, \bar{s}^{\prime}\right\}, T=\{t, \bar{t}\}, T^{\prime}=\left\{t^{\prime}, \bar{t}^{\prime}\right\}$. By renaming the roots within these pairs if necessary, we have

$$
r=s+s^{\prime}=\bar{s}+\bar{s}^{\prime}=t+t^{\prime}=\bar{t}+\bar{t}^{\prime} .
$$

Given a system $r_{1}, \cdots, r_{n}$ of fundamental roots for $\Sigma$, the height of a root $\sum_{i=1}^{n} z_{i}{ }^{r}{ }_{i}$ is defined to be $\sum_{i=1}^{n} z_{i}$ ( $z_{i}$ integers $)$. Since the height is additive, all roots in $(*)$ are positive.

Suppose $\Sigma$ has type $A_{n}, r=r_{1}+\cdots+r_{n}$. We may assume $S=r_{1}+\cdots+r_{m}$, $s^{\prime}=r_{m+1}+\cdots+r_{n}, t=r_{1}+\cdots+r_{k}, t^{\prime}=r_{k+1}+\cdots+r_{n}, 1 \leq m<k<n$. Let $w^{\prime}$ be the reflection corresponding to the root $r_{m+1}+\cdots+r_{k}, w^{\prime \prime}$ the reflection corresponding to $r_{n=k+1}+\cdots+r_{n-m+p^{*}}$ Then $w=w^{\prime} w^{\prime \prime} \in W$ and $w(s)=t$, $w\left(s^{\prime}\right)=t^{\prime}, w(\bar{s})=\bar{t}, w\left(\bar{s}^{\prime}\right)=\bar{t}^{\prime} . w(S)=T, w\left(S^{\prime}\right)=T^{\prime}$ follows.

A similar argument works for $\Sigma$ of type $E_{6}$ and $D_{n}, n \geq 4$. In fact, for $D_{n}$, note that $\left\{S, S^{\prime}\right\}=\left\{T, T^{\prime}\right\}$ always.

Lemma 2. If ${ }^{2} \Sigma$ bas type $F_{4}$ or $B_{n-1}$, and $R=S+S^{\prime}=T+T^{\prime}$, where all these roots are long, there is a w $w$ with $w(S)=T, w\left(S^{\prime}\right)=T^{\prime}$.

Proof. By direct examination, as in previous lemma.

Remark. The hypothesis is never satisfied for ${ }^{2} \Sigma$ of type $C_{n}$.

We shall repeatedly use the following without comment.

Lemma 3. Suppose $K_{1}, K_{2}$ are fields and $f: K_{1} \times K_{2} \rightarrow A$ is a map into an 
abelian group satisfying (a) (resp. (b)).

(a) $f\left(t+t^{\prime}, u\right)=f(t, u) f\left(t^{\prime}, u\right)$, all $t, t^{\prime} \in K_{1}, u \in K_{2}$,

(b) $f\left(t, u+u^{\prime}\right)=f(t, u) f\left(t, u^{\prime}\right)$, all $t \in K_{1}, u, u^{\prime} \in K_{2}$,

then if $f(t, u)=f(\lambda t, u)$ for some $\lambda \neq 0,1$ in $K_{1}$ and for all $t \in K_{1}, u \in K_{2}$ (resp. $f(t, u)=f(t, \mu u), u \neq 0,1$ in $K_{2}$, all $\left.t, u\right)$, then $f$ is identically 1 .

Proof. $1=f((\lambda-1) t, u)$, for all $t, u$. But $(\lambda-1) t$ ranges over $K_{1}^{\times}$as $t$ does, implying $f \equiv 1$. The alternate case is similar.

We call an $f$ satisfying (a) and (b) biadditive.

The 3-dimensional unitary groups ${ }^{2} A_{2}(q)$. The result of this section is that the multiplier of $G=\operatorname{SU}(3, q)$ is trivial. See [10] for the information below about $G$.

Let $G=\mathrm{SU}(3, q), q=p^{n}, K=\mathrm{GF}\left(q^{2}\right), K_{0}=\mathrm{GF}(q),\langle\tau\rangle=\mathrm{Gal}\left(K / K_{0}\right), \lambda^{\tau}=\lambda^{q}$, $\lambda \in K$. $G$ is the subgroup of $\operatorname{SL}\left(3, q^{2}\right)$ stabilizing the Hermitian form whose matrix is

$$
\left(\begin{array}{lll}
0 & 0 & 1 \\
0 & 1 & 0 \\
1 & 0 & 0
\end{array}\right)
$$

$$
\begin{aligned}
& |\operatorname{SU}(3, q)|=q^{3}\left(q^{3}+1\right)\left(q^{2}-1\right), \quad\left|\operatorname{SL}\left(3, q^{2}\right)\right|=q^{6}\left(q^{6}-1\right)\left(q^{4}-1\right), \\
& |\operatorname{SL}(2, q)|=q\left(q^{2}-1\right) .
\end{aligned}
$$

The special p-groups $Q_{1}=\left\{x(a, b) \mid b+b^{\tau}+a a^{\prime}=0, b, a \in K\right\}, Q_{2}=\{y(a, b) \mid$ $\left.b+b^{\tau}+a a^{\tau}=0, b, a \in K\right\}$ form Sylow $p$-subgroups of $G$, where

$$
x(a, b)=\left(\begin{array}{ccc}
1 & a & b \\
0 & 1 & -a^{\tau} \\
0 & 0 & 1
\end{array}\right), \quad y(a, b)=\left(\begin{array}{rrr}
1 & 0 & 0 \\
a^{\tau} & 1 & 0 \\
b & -a & 1
\end{array}\right) .
$$

Their normalizers are generated by $Q_{i}$ and the cyclic group $H, H=\{b(\lambda) \mid \lambda \in K$, $\lambda \neq 0\}$, where

$$
b(\lambda)=\left(\begin{array}{lll}
\lambda^{-\tau} & 0 & 0 \\
0 & \lambda^{\tau-1} & 0 \\
0 & 0 & \lambda
\end{array}\right) \quad \begin{aligned}
& b(\lambda)^{-1} x(a, b) b(\lambda)=x\left(\lambda^{2 \tau-1} a, \lambda^{\tau+1} b\right), \\
& b(\lambda)^{-1} y(a, b) b(\lambda)=y\left(\lambda^{\tau-2} a, \lambda^{-\tau-1} b\right) .
\end{aligned}
$$

The groups $Z_{1}=\{x(0, b)\} \subseteq Q_{1}, Z_{2}=\{y(0, b)\} \subseteq Q_{2}$ satisfy $Z_{i}=Q_{i}^{\prime}=Z\left(Q_{i}\right)$ and $S=\left\langle Z_{1}, Z_{2}\right\rangle \cong \operatorname{SL}(2, q)$. $S$ is normalized by $H,|H \cap S|=q-1,|H S|=$ $q\left(q^{2}-1\right)(q+1)$. The subgroup of $H$ of order $q+1$ centralizes $Z_{1}, Z_{2}$, hence $S$.

To prove $m(G)=1$, we prove $m_{r}(G)=1$ for all primes $r$ dividing $|G|$.

The index of $H S$ in $G$ is $q^{2}\left(q^{2}-q+1\right)$. It is straightforward to verify that 


$$
(|H S|, G: H S \mid)=\left\{\begin{array}{rrrrr}
(\mathrm{i}) & 3 q & p \neq 3 & q \equiv 2 & (\bmod 3) \\
\text { (ii) } & q & p \neq 3 & q \equiv 1 & (\bmod 3) \\
\text { (iii) } & q & p=3 & &
\end{array}\right.
$$

Consider a central extension $\widetilde{H S}$ of $H S$ by a cyclic group $A$ of prime order $r$, $r \neq p . S=\operatorname{SL}(2, q)$ is generated by its p-elements, and $r \nmid m(S)$ [13]. So, the induced extension $\tilde{S}$ of $S$ splits. Now, $S \cong S_{0} \triangleleft H S$, where $S_{0}$ is the characteristic subgroup of $\tilde{S} \triangleleft \tilde{H S}$ generated by the $p$-elements of $\tilde{S} ; \tilde{S}=S_{0} \times A$. Since $H S / S$ is cyclic, $A \not \underline{(H S)^{\prime}}$. Thus, $m_{r}(H S)=1$, for all $r \neq p$.

If $r \neq p$ is a prime, $r|| G: H S|, r \nmid| H S \mid$, then a Sylow $r$-subgroup $R$ of $G$ is cyclic. In fact, for such $r, R$ is a Sylow $r$-subgroup of $\operatorname{SL}\left(3, q^{2}\right) \supset G$, and $R$ is contained in a cyclic Singer subgroup [10] of $\operatorname{SL}\left(3, q^{2}\right)$. So, $m_{r}(G)=1$ for such $r$.

Now assume $r$ is a prime, $r|| H S \mid, r \neq p$. If (i) holds and $r \neq 3$, or (ii) or (iii) holds, a Sylow $r$-subgroup of $G$ is contained in $H S$. But $m_{r}(H S)=1$ implies $m_{r}(G)=1$.

We next treat separately the more difficult cases (I): $r=3, p \neq 2, q \equiv 2$ $(\bmod 3)$; and $(\mathrm{II}): r=p$. Note that in case $(\mathrm{I}), Z(G)$ has order 3 .

(I) If $T$ is a Sylow 3-subgroup of $H S, T=T_{1} \times T_{2}, T_{1} \cong T_{2} \cong Z_{3 m}, 3^{m}=$ $(q+1)_{3}$. $T$ has index 3 in $P$, a Sylow 3 -subgroup of $G$, because (i) applies. So, $|P|=3^{2 m+1}$, and $Z(G)=\Omega_{1}\left(T_{2}\right), T_{2} \subseteq H, T_{1} \subseteq S$.

Let $x \in N_{S}\left(T_{1}\right)$ where $x$ inverts $T_{1}$. Then $x$ centralizes $T_{2}$, and $|x|=4$ if $q$ is odd, $|x|=2$ if $q$ is even. Since $1 \neq Z(G) \subset P, P$ is nonabelian, by (2).

We now look at the subgroup $N=N_{G}(T)$, which contains a Sylow 3-subgroup of $G$. The normal subgroup $N_{0}$ of $N$, consisting of elements which induce automorphisms of determinant 1 on the vector space $T / \Phi(T)$, has index 2 in $N$, since $x \in N \backslash N_{0}$. By the Frattini argument, a Sylow 3-subgroup $P$ of $G$ is normalized by $x_{1} \in x N_{0}$. Replacing $x_{1}$ by an odd power of $x_{1}$, we may assume $x_{1}$ is a 2-element, acting with eigenvalues $\{1,-1\}$ on $T / \Phi(T)$. $x_{1}$ also acts on $P / T \cong Z_{3}$. Since $x_{1}$ permutes an odd number of pairs $\left\{u, u^{-1}\right\}=T y \cup T y^{-1}, y \in P \backslash T$, we may assume $y^{x_{1}}$ equals $y$ or $y^{-1}$. We assume $y^{x_{1}}=y$ and derive a contradiction. Set $T=$ $V_{1} \times V_{2}, V_{1}=\left[T, x_{1}\right], V_{2}=C_{T}\left(x_{1}\right) ; V_{1} \cong V_{2}$ are cyclic, generated by $v_{1}, v_{2}$, respectively. Since $x_{1}$ and $y$ commute, $y$ normalizes $V_{1}$ and $V_{2}$. As $y^{3} \in T$, abelian, $y$ effects automorphisms of orders 1 or 3 on $V_{1}$ and $V_{2}$. So, $\left[V_{i}, y\right] \subseteq$ $\Omega_{1}\left(V_{i}\right), i=1,2$. If $\left\langle V_{2}, y\right\rangle$ were cyclic, then $Z(G)=\Omega_{1}\left(V_{2}\right)$ is not contained in $P^{\prime}$. By a transfer lemma $(2), Z(G) \nsubseteq G^{\prime}$, a contradiction to $G$ having no nontrivial factor group. So, the noncyclic group $\left\langle V_{2}, y\right\rangle$ contains an elementary abelian subgroup $E$ of order $3^{2}$. Since $\Omega_{1}\left(V_{1}\right) \subseteq Z(P),\left\langle\Omega_{1}\left(V_{1}\right), E\right\rangle$ is elementary abelian subgroup $E$ of order $3^{3}$, an impossibility in $G \subset \operatorname{SL}\left(3, q^{2}\right)$. Thus, $y^{x_{1}}=y^{-1}$, and $y^{3} \in V_{1}$.

Let $\widetilde{G}$ be an extension of $G$ by $\langle\alpha\rangle=A \cong Z_{3} . \widetilde{R}$ denotes the induced extension of $R \subseteq G$, and $\tilde{g}$ belongs to the coset $g \in \widetilde{G}=G / A$. To show $\tilde{G}$ splits, it suffices to show $\widetilde{P}$ splits. 
First, we show $A \nsubseteq \underline{T^{\prime}}$. If $a \in \tilde{T}^{\prime}, a^{ \pm 1}=\left[\tilde{v}_{1}, \tilde{v}_{2}\right]_{\sim}$ But, modulo $A, x_{1}$ inverts $v_{1}$, centralizes $v_{2}$ and so inverts $\alpha$, contrary to $a \in Z(G)$. Next, if $\langle\zeta\rangle=Z(G)=$ $\Omega_{1}\left(T_{2}\right)$, we show $\tilde{\zeta}^{3}=1$. Since $\langle\zeta\rangle \subseteq P^{\prime}$ and $T$ is abelian, $\zeta=[t, y]$, some $t \in T$. As $G$ is perfect, $\tilde{\zeta}$ is central in $\tilde{G}$. So, if $\tilde{\zeta} \alpha^{i}=[\tilde{t}, \tilde{y}]$, bilinearity of the commutator and $\tilde{y}^{3} \in \tilde{T}$, abelian, imply $\left(\tilde{\zeta} \alpha^{i}\right)^{3}=\left[\tilde{t}, \tilde{y}^{3}\right]=1$ and $\tilde{\zeta}^{3}=1$. This means $\tilde{T}$ splits over $A: \tilde{T}=T_{0} \times A, T_{0}$ is $\tilde{x}_{1}$-invariant and $T_{0}=V_{01} \times V_{02}, V_{01}=\left[T_{0}\right.$, $\left.\tilde{x}_{1}\right]=\left\langle v_{01}\right\rangle, V_{02}=C_{T_{0}}\left(\tilde{x}_{1}\right)=\left\langle v_{02}\right\rangle$. Consider the action of $\left\langle x_{1}, y\right\rangle$ on $\bar{T}=\tilde{T} / \Phi(\tilde{T})$ (note $\Phi(\widetilde{T})=\Phi\left(T_{0}\right)$ ). Denoting images of $\tilde{T} \rightarrow \bar{T}$ by - , we have $\bar{T}=\left\langle\bar{v}_{01}\right\rangle \times$ $\left\langle\bar{v}_{02}\right\rangle \times\langle\bar{a}\rangle$, and the factors are $\tilde{x}_{1}$-invariant. Replacing, if necessary, $\tilde{y}$ by a power of $\left[y, \tilde{x}_{1}\right] \in y^{-2}$ we may assume. $\tilde{y}^{\tilde{x}}=\tilde{y}^{-1}$; then $\tilde{y}^{3} \in V_{01}$. If $\tilde{y}$ acts trivially on $\bar{T}$, then $\tilde{P}$ splits, i.e., $\left\langle T_{0}, \tilde{y}\right\rangle$ is a complement to $\langle\alpha\rangle$ in $\widetilde{P}$. If $\tilde{y}$ acts nontrivially, we get a splitting as above, provided we show $\bar{\alpha} \notin[\bar{T}, \tilde{y}]$. If $\bar{\alpha} \in[\bar{T}, \tilde{y}]$, then $\bar{\alpha}=\left[\bar{v}_{01}^{i} \bar{v}_{02}^{j}, \tilde{y}\right]=\left[\bar{v}_{01}, \tilde{y}\right]^{i}\left[\bar{v}_{02}, \tilde{y}\right]^{j}$. Conjugating both sides by $\tilde{x}$, the effect of $\tilde{x}$ on the various elements forces $\left[\bar{v}_{02}, \tilde{y}\right]^{j}=\overline{1}$. If $i \neq 0(\bmod 3)$, then in $P$, $\left[V_{1}, y\right] \subseteq \Phi(T)=\mho^{1}(T)$. Consequently, $\left[\Omega_{1}\left(V_{1}\right), y\right]=1$ and $\left[\Omega_{1}(T), y\right]=1$. Now, we claim, $\left\langle y^{3}\right\rangle$ is a proper subgroup of $V_{1}$. If $\left\langle y^{3}\right\rangle=V_{1},\left[V_{1}, y\right]=1$. Since $y$ is nontrivial on $T / \Phi(T)$, this forces $\left[v_{2}, y\right] \equiv v_{1}^{k}(\bmod \Phi(T)), k \neq 0(\bmod 3)$. Hence $\left[\Omega_{1}\left(V_{2}\right), y\right]=\Omega_{1}\left(V_{1}\right) \neq 1$, absurd, as $\Omega_{1}\left(V_{2}\right)=Z(G)$. By this claim, we may choose $w \in\left\langle v_{1}\right\rangle$ with $w^{3}=y^{3}$. Then $\left(y w^{-1}\right)^{3}=1$ since $[w, y] \in \Omega_{1}\left(V_{2}\right)=Z(G)$. So, $\left\langle y w^{-1}, \Omega_{1}(T)\right\rangle$ is elementary abelian of order $3^{3}$, contradiction. Therefore, $i \equiv 0$ (mod 3), implying $\bar{\alpha} \notin[\bar{T}, \tilde{y}]$. This proves $m_{3}(G)=1$.

(II) Let $\widetilde{G}$ be a central extension of $G$ by a $p$-group $A$. Denote by $\widetilde{Q}$ the induced extension of $Q$, by $\overrightarrow{b(\lambda)}$ a representative in $\widetilde{G}$ for $b(\lambda) \in G$. Assume $A \subseteq \widetilde{Q}^{\prime}$. To show $m_{p}(G)=1$, we show $\overrightarrow{b(\lambda)}$, for $\lambda$ a generator of $K^{\times}$, has no eigenvalue 1 on the Lie algebra $L(Q)$ associated with $Q$ (unless $G=\mathrm{SU}(3,2)$ in which case $m_{2}(G)=1$ as $Q \cong Q_{8}$ the quaternion group). We will then be done by a transfer lemma (2).

$$
\begin{array}{ll}
L(Q)=L_{1} \oplus L_{2}, & L_{1}=Q / Q^{\prime}, \quad L_{2}=Q^{\prime}, \\
L(\tilde{Q})=\tilde{L}_{1} \oplus \tilde{L}_{2} \oplus \tilde{L}_{3}, & \tilde{L}_{1} \simeq L_{1} \text { as } \widetilde{b(\lambda)} \text {-groups, }
\end{array}
$$

and these first terms generate $L(\widetilde{Q}), L(Q)$ respectively, as rings. The eigenvalues for $\overparen{b(\lambda)}$ on $L_{1}$ are $\left\{\lambda^{(2 q-1) a} \mid \alpha=1, p, p^{2}, \cdots, p^{2 n-1}\right\}$ and those on $L_{2}$ are $\left\{\lambda^{(q+1)} \beta \mid \beta=1, p, \cdots, p^{n-1}\right\}$.

If 1 were to occur as an eigenvalue on $\tilde{L}_{2}$, then $i=\lambda^{(2 q-1)\left(\alpha_{+} \alpha^{\prime}\right)}$. We may assume $a^{\prime}=1$. The exponent must satisfy $(\alpha+1)(2 q-1) \equiv 0\left(\bmod q^{2}-1\right)$.

Now, $\left(2 q-1, q^{2}-1\right)=(2 q-1, q+1)=1$ or 3 . In the first case, $q^{2}-1 \mid a+1$ $=p^{i}+1 \cdot p^{i}<q^{2}$ forces $i=2 n-1$ and $p^{2 n}-1=p^{2 n-1}+1$; hence $q=2$, the case we eliminated right away. In the second case $q^{2}-1 \mid 3(\alpha+1)=3\left(p^{i}+1\right)$, or $p^{2 n}-1 \leq 3 p^{i}+3, p^{2 n}-3 p^{i} \leq 2, p^{i}\left(p^{2 n-i}-3\right) \leq 2$. If $p^{2 n-i}-3 \leq 0$, then $1<p^{2 n-i} \leq 3$. So, $p^{2 n-i}=2\left(p^{2 n-i}=3\right.$ is out since $\left.3 \mid q+1\right)$. Thus $q^{2}=2 p^{i}$, or 
$i=2 n-1 . q^{2}-1 \mid 3\left(p^{i}+1\right)$ implies $2 p^{i}-1 \mid 3 p^{i}+1=\left(2 p^{i}-1\right)+p^{i}+2$, and $2 p^{i}-$ $1 \leq p^{i}+2$, or $p^{i} \leq 3$. Hence, $p^{i}=2$ and $q=2$, and we are done as before. If $p^{2 n-i}-3>0$ and $p^{i}=1$, then $p^{2 n-i}-3=1$ or 2 , and $1 \neq p^{2 n-i}=4$ or 5 . But $q^{2}=$ $p^{i} p^{2 n-i}=4$ or 5 forces $q=2$, done. If $p^{2 n-i}-3>0$ and $p^{i} \neq 1$, then $p^{i}=2$ and $p^{2 n-i}=4$. But $q^{2}=p^{i} p^{2 n-i}=8$ is absurd. Thus the eigenvalue 1 does not occur on $L_{2}$.

If 1 were to occur as an eigenvalue on $\widetilde{L}_{3}$, we must have $1=\lambda^{(2 q-1) a} \lambda^{(q+1) \beta}$, or $(2 q-1) \alpha+(q+1) \beta \equiv 0 \bmod \left(q^{2}-1\right)$. We may assume $\alpha=1$. Then $q-1 \leq$ $(2 q-1)+(q+1) p^{i}=\left(2+p^{i}\right) q+\left(p^{i}-1\right)$, where $\beta=p^{i}<q$. So, $q\left(q-p^{i}-2\right) \leq p^{i}$, which forces $q-p^{i}-2 \leq 0, q \leq p^{i}+2$, and $q=2,3$, or $4, i=0,1 . q=2$ is out, and $2 q-1+(q+1) p^{i} \equiv 0 \bmod \left(q^{2}-1\right)$ cannot be satisfied for $q=3$ or 4 . So, 1 does not occur on $\tilde{L}_{3}$.

This proves $m_{p}(G)=1$, the last step to proving $m(G)=1$.

The unitary groups ${ }^{2} A_{n}(q), n \geq 5, n$ odd. $\Sigma$ denotes a root system of type $A_{n}, n \geq 5, n$ odd, with Dynkin diagram

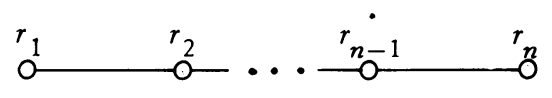

${ }^{2} \Sigma$ has type $C_{m+1}, n=2 m+1$, with a set of fundamental roots $S_{1}=\left\{r_{1}, r_{n}\right\}, S_{2}=$ $\left\{r_{2}, r_{n-1}\right\}, \cdots$ (short), and $S_{m+1}=\left\{r_{m+1}\right\}$ (long).

(i) $\left[x_{R}(t), x_{S}(u)\right]=1 \quad R, S$ long,

(ii) $\quad=1 \quad R$ long, $S$ short,

(B)

(iii) $\quad=1 \quad R, S$ short,

(iv) $\quad=x_{R+S}(\epsilon t u) \quad R, S R+S$ short, $\epsilon= \pm 1$,

(v) $\quad=x_{R+S}(\epsilon(t \bar{u}+\bar{t} u)) \quad R, S$ short, $R+S$ long, $\epsilon= \pm 1$,

(vi) $\quad=x_{R+S}(\epsilon t u) x_{R+2 S}(\eta t u \bar{u}) R$ long, $S$ short, $\epsilon= \pm 1, \eta= \pm 1$.

The relations (A). Set $Y_{R} / A=X_{R}$, all $R \epsilon^{2} \Sigma$. We claim that $Y_{R}$ is abelian. If $R$ is long, we may assume $R$ is a root of maximal height, i.e., $R=\left\{r_{1}+r_{2}+\cdots+\right.$ $\left.r_{n}\right\}$. Then $X_{R}=\left[X_{S}, X_{T}\right] \subseteq U^{\prime}$ where $S=\left\{r_{1}+r_{2}+\cdots+r_{m}, r_{m+2}+\cdots+r_{n}\right\}$, $T=\left\{r_{1}+\cdots+r_{m+1}, r_{m+1}+\cdots+r_{n}\right\}$. Since $X_{R} \subseteq Z(U), Y_{R} \subseteq Z_{2}(V) \cap A V^{\prime}$. So, $Y_{R}$ is abelian. If $R$ is short, we may assume $R=S$ (above). Then $X_{R}=$ $\left[X_{s_{1}}, X_{Q}\right], Q=\left\{r_{2}+\cdots+r_{m}, r_{m+2}+\cdots+r_{n-1}\right\}$ and $X=X_{S_{1}} X_{Q} X_{R}$ is a group of class 2. So, for $Y / A=X, Y$ has class 2 or 3 , and $Y_{R} \subseteq A Y^{\prime}$ is abelian. By Fitting's lemma, $Y_{R}=C_{Y_{R}}(H) \oplus\left[Y_{R}, H\right], H$ the Cartan subgroup.

If $R$ is short, or if $q>2$ and $R$ is long, $H$ is nontrivial on $X_{R}$. Define $y_{R}(t)$ as the unique element of $x_{R}(t) \cap\left[Y_{R}, H\right]$. If $R$ is long and $q=2$, choose short 
roots $S, T$ with $S+T=R$. Define $y_{R}(1)=\left[y_{S}(\omega), y_{T}(1)\right]$, where we choose some $\omega \neq 1$. Taking $R, S, T$ as in the previous paragraph, $X_{S}, X_{T} \subseteq U^{\prime}$ since $n \geq 5$. So, $\left[Y_{R}, Y_{S}\right]=\left[Y_{R}, Y_{T}\right]=1$.

Define $y_{R}(0)=y_{R}(1)^{2}=1$. In (v), we will see that this is independent of $\omega$ (we do not need independence until after (v)). Then, by Lemma 1, it is independent of $S$ and $T$.

These representatives satisfy (C) and (I).

The relations (B). (i) $\left[y_{R}(t), y_{S}(u)\right]=f(t, u)$. We may assume that $R$ is a root of maximal height and that $S$ is positive: $S=\left\{r_{j}+\cdots+r_{n-j+1}\right\}, 1<j \leq m+1$. We may assume that $j \leq m$ upon conjugating the equation by $n_{s_{m}}$ if $j=m+1$. Then, $X_{S}=\left[X_{Q}, X_{T}\right] \subseteq U^{\prime}$, where $Q=\left\{r_{j}, r_{n-j+1}\right\}, T=\left\{r_{j+1}+\cdots+r_{n-j+1}\right.$, $\left.r_{j}+\cdots+r_{n-j}\right\}$. So, $Y_{R} \subseteq Z_{2}(V), Y_{S} \subseteq A V^{\prime}$, which implies $\left[Y_{R}, Y_{S}\right]=1$, i.e., $f \equiv 1$.

(ii) $\left[y_{R}(t), y_{S}(u)\right]=f(t, u)$. We may assume $R$ is a root of maximal height and $S$ positive. $S=\left\{r_{j}+\cdots+r_{k}, r_{n-k+1}+\cdots+r_{n-j+1}\right\}$ for $k \leq m$ or $k \geq m+2$; $j \leq m, j<n-k+1$. If $k \geq m+2$, by applying $n_{Q}$ (which fixes $R$ ) to $S$, where $Q=\left\{r_{n-k+1}+\cdots+r_{k}\right\}$, we may assume $k \leq m$. Applying $b_{S_{k}}(\lambda)$ to our relation gives $f(t, u)=f\left(t, \lambda^{-1} u\right), \lambda \in K$. Since $f$ is biadditive and $|K| \geq 4, f \equiv 1$.

(iii) $\left[y_{R}(t), y_{S}(u)\right]=f(t, u)$. Clearly, $f$ is biadditive. We may assume $R=S_{1}$. Then $S=\left\{r_{1}+\cdots+r_{j}, r_{n-j+1}+\cdots+r_{n}\right\}, j \neq m+1, n,\left\{r_{j}+\cdots+r_{k}, r_{n-k+1}+\right.$ $\left.\cdots+r_{n-j+1}\right\}, 3 \leq j \leq m, k \neq n-j+1, n-1$, or $-\left\{r_{j}+\cdots+r_{k}, r_{n-k+1}+\cdots+\right.$ $\left.r_{n-j+1}\right\}, 2 \leq j \leq m, k \neq n-j+1$. We treat only the first possibility for $S$. If $j>2$, conjugation by $b_{s_{j}}(\lambda)$ gives $f(t, u)=f\left(t, \lambda^{2} u\right)$. Biadditivity and $|K| \geq 4$ gives $f \equiv 1$. If $j=2, m \geq 3$, conjugation by $b_{s_{3}}(\lambda)$ gives the same conclusion. Assume $j=2, m=2, n=5$. We have $Q+T=S, Q=\left\{r_{1}+r_{2}+r_{3}+r_{4}, r_{2}+r_{3}+\right.$ $\left.r_{4}+r_{5}\right\}, T=-\left\{r_{2}+r_{3}, r_{3}+r_{4}\right\}$, and $\left[y_{Q}(u), y_{T}(\epsilon)\right]=y_{S}(u) a, a \in A$, by (iv). Conjugating the left side by $y_{R}(t)$, we get $\left[y_{Q}(u) y_{Q+R}(\eta(u \bar{t}+\bar{t} u)), y_{T}(\epsilon)\right]=\left[y_{Q}(u)\right.$, $\left.y_{T}(\epsilon)\right], \eta= \pm 1, \epsilon= \pm 1$, by (v), (ii) and the commutator identities. So, $y_{R}(t)$ commutes with the right side too, hence with $y_{S}(u)$; thus, $f \equiv 1$ here. The second and third cases are handled similarly.

(iv) $\left[y_{R}(t), y_{S}(u)\right]=y_{R+S}(\epsilon t u) f(t, u)$. By (iii), $f$ is biadditive. We may assume $R=S_{1}$. Then, $S=\left\{r_{2}+\cdots+r_{k}, r_{n-k+1}+\cdots+r_{n-1}\right\}$, or $-\left\{r_{1}+\cdots+r_{k}\right.$, $\left.r_{n-k+1}+\cdots+r_{n}\right\}$. We consider the former case, the latter being similar. As in (ii), we may assume $k \leq m$. We can get $f \equiv 1$ if $n \geq 7$ upon conjugating the relation by $b_{Q}(\lambda)$ where $Q=S_{k}$ if $k>2$, or $Q=S_{k+1}$ if $k=2$. For $n=5$, conjugation by $b_{s_{2}}(\lambda)$ gives $f(t, u)=f\left(\lambda^{-1} t, \lambda^{2} u\right)$. Conjugation by $b_{s_{3}}(\mu)$ gives $f(t, u)=$ $f\left(t, \mu^{-1} u\right), \mu \in K_{0}^{\times}$. This implies $f \equiv 1$ if $q>2$. For $q=2$, all we can say for now is that $\langle f(t, u) \mid t, u \in K\rangle$ is a subgroup of a four-group. The next section treats the case $q=2$.

(v) $\left[y_{R}(t), y_{S}(u)\right]=y_{R+S}(\epsilon(t \bar{u}+\bar{t} u)) f(t, u)$. By (ii), $f$ is biadditive. We may 
assume $R=S_{1}, S=\left\{r_{1}+\cdots+r_{n-1}, r_{2}+\cdots+r_{n}\right\}$ or $-\left\{r_{1}+\cdots+r_{k}, r_{n-k+1}+\right.$ $\left.\cdots+r_{n}\right\}$; we treat the first case only. Conjugating the relation by $b_{R}(\lambda), f(t, u)=$ $f\left(\lambda^{2} t, \lambda \lambda^{-1}\right)$. Applying $b_{s_{2}}(\lambda), f(t, u)=f\left(\lambda^{-1} t, \bar{\lambda} u\right)$. So, $f(t, u)=f(t, \lambda \bar{\lambda} u)$. By (ii) and (I), if $q>2, f$ is biadditive, which gives $f \equiv 1$. Let $q=2, Q=\left\{r_{2}+\cdots\right.$ $\left.+r_{n-2}\right\}, K=\left\{0,1, \omega, \omega^{2}\right\}$. Since $x_{R}(t)^{n} Q=x_{S}(t), x_{S}(u)^{n} Q=x_{R}(u)$, we have $f(t, u)=f(u, t)$. Depending on how we defined $y_{R+S}(1)$, either $f(\omega, 1)$ or $f\left(\omega^{2}, 1\right)$ is 1 , but the choice need not be specified for this argument. Using (ii) and the above,

$$
\begin{aligned}
f(1,1) & =\left[y_{R}(1), y_{S}(1)\right]=\left[y_{R}\left(\omega+\omega^{2}\right), y_{S}(1)\right]=y_{R+S}(1)^{2} f(\omega, 1) f\left(\omega^{2}, 1\right) \\
& =y_{R+S}(1)^{2} f\left(1, \quad \omega^{2}\right)^{2}=\left[y_{R}(1), y_{S}\left(\omega^{2}\right)^{2}\right]=1 .
\end{aligned}
$$

Consequently, $\left[y_{R}(\omega), y_{S}(1)\right]=\left[y_{R}(1+\omega), y_{S}(1)\right]=\left[y_{R}\left(\omega^{2}\right), y_{S}(1)\right]$, so $f(\omega, 1)=$ $f\left(\omega^{2}, 1\right)=1$ also. This clears up the ambiguity in the choice of $y_{T}(1), T$ long. So, $f \equiv 1$.

(vi) $\left[y_{R}(t), y_{S}(u)\right]=y_{R+S}(\epsilon t u) y_{R+2 S}(\eta t u \bar{u}) f(t, u)$. Using previous relations and the commutator identities, $f(t, u)$ is biadditive. We may assume $S=S_{1}$; then $R=\left\{r_{2}+\cdots+r_{n-1}\right\}$ or $-\left\{r_{1}+\cdots+r_{n}\right\}$. We treat the first case only. Conjugating by $b_{s_{1}}(\lambda)$ gives $f(t, u)=f\left(\lambda^{-1} \bar{\lambda}^{-1} t, \lambda^{2} u\right)$. Using $b_{s_{2}}(\mu), f(t, u)=f\left(\mu \bar{\mu} t, \mu^{-1} u\right)$. Thus, $f(t, u)=f(t, \lambda u)$, and $f \equiv 1$ as $|K| \geq 4$.

The exceptional case ${ }^{2} A_{5}(2)=\mathrm{PSU}_{6}(2)$. As shown in the previous section, the only relations which cannot be lifted to the $y_{R}(t)$ are of type (iv). Hence, $A$ is generated by all $f(t, u)$ of this type. Since any triple $\{R, S, R+S\}$ of this type is conjugate under the Weyl group (an easy verification), the $f(t, u)$ for a fixed $R, S$ generate $A$. Now, let $X$ be the group generated by $X_{R}, X_{S}, X_{R+S}, X_{-R}$, $X_{-S}, X_{-R} S^{\circ} X$ maps onto the simple group $A_{2}(4)$ which has the Chevalley commutator relations as those holding among the $x_{R}(t), x_{S}(u), \cdots$ (rewriting $x_{R}(t)=$ $x_{r}(t) x_{\bar{r}}(\bar{t})$ as $x_{R}(\bar{t})$ if necessary) and $Z(X)=\left\langle b_{R}(\lambda) b_{S}\left(\lambda^{-1}\right)\right\rangle \cong Z_{3}$. Let $Y / A=X$. By construction, $y_{R}(t), y_{S}(u), \cdots \in Y^{\prime}$; hence $A \subseteq Y^{\prime}$. Since $M_{2}(X) \cong Z_{4} \times Z_{4}$ [8] and $f(t, u)^{2}=1, A$ is a subgroup of a four-group.

One could prove that $A$ is a four-group by constructing a stable cocycle. However, in the group $M(22)$ discovered by Fischer [6], the centralizer of a 3 transposition is a perfect extension of $U_{6}(2)$ by $Z_{2}$. Hence, 2||$A \mid$. In the determination of $m_{2}(M(22))$ (see [9]), it is seen that 4||$A \mid$, forcing $|A|=4=m_{2}\left({ }^{2} A_{5}(2)\right)$. An alternative proof is to note that the diagonal outer automorphism $d$ of order 3 on ${ }^{2} A_{5}(2)$ induces a diagonal outer automorphism $d$ on $X$. Since the action of $d$ on cohomology commutes with the restriction map and $d$ acts fixed-point-freely on $M_{2}(X), d$ acts fixed point freely on the image of the restriction $A=H^{2}\left({ }^{2} A_{5}(2), C^{\times}\right)$ $\rightarrow H^{2}\left(X, \mathbf{C}^{\times}\right)$. The last paragraph implies that the restriction is injective. 
So, $|A|=4$ as $2 \leq|A| \leq 4$ and $d$ cannot be fixed point free on a group of order 2 .

The unitary groups ${ }^{2} A_{n}(q), n$ even, $n \geq 4$. $\Sigma$ denotes a root system of type $A_{n}, n=2 m$, with Dynkin diagram

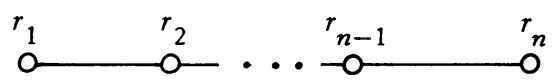

${ }^{2} \Sigma$ is a root system of type $C_{m}$ having as a set of fundamental roots $S_{1}=\left\{r_{1}\right.$, $\left.r_{n}\right\}, \cdots S_{m-1}=\left\{r_{m-1}, r_{m+2}\right\}$ (short) and $S_{m}=\left\{r_{m}, r_{m+1}, r_{m}+r_{m+1}\right\}$ (long). The "one-parameter subgroups" $X_{R}$ are abelian of order $q^{2}$ if $R$ is short, nonabelian of class two of order $q^{3}$ if $R$ is long.

We let $\Gamma \cong \mathrm{SU}(n+1, q) ; \Gamma / Z(\Gamma) \cong G . \Gamma$ is generated by elements $x_{R}(t)$, $x_{R}(u, v)$ satisfying the relations below. We let $\hat{G}$ be a perfect extension of $\Gamma$ by $M_{p}(\Gamma)$ and lift these generators and relations to $\hat{G}$.

$$
x_{R}(t) x_{R}(u)=x_{R}(t+u) \quad R \text { short, } t, u \in K \text {, }
$$

$\left[x_{R}(t, u), x_{R}(v, w)\right]=x_{R}(0, \epsilon(t \bar{v}-\bar{t} v))$,

$R$ long, $R=\{r, \bar{r}, r+\bar{r}\}$, $\epsilon=N_{r \bar{r}}= \pm 1$, $x_{R}(t, u) x_{R}(v, w)=x_{R}(t+v, u+w-\epsilon \overline{t v})$.

$$
\left[x_{R}(t), x_{S}(u)\right]=1
$$

$R, S$ short,

$$
=x_{R+S}(\epsilon t u)
$$

$$
=x_{R+S}(0, \epsilon(t \bar{u}-\overline{t u}))
$$

$R, S, R+S$ short,

$\epsilon= \pm 1$,

$R, S$ short,

$R+S$ long,

(iv) $\left[x_{R}(t, u), x_{S}(v, w)\right]=x_{1 / 2(R+S)}(\epsilon t v)$

$\epsilon= \pm 1$,

$R, S$ long,

$1 / 2(R+S)$ short,

$\epsilon= \pm 1$.

(v) $\quad\left[x_{R}(t, u), x_{S}(v)\right]=1$

$R$ long, $S$ short,

$$
\begin{aligned}
& =x_{R+S}(\epsilon \bar{u} v) x_{R+2 S}(\eta t v, \delta u v \bar{v}) R, R+2 S \text { long, } \\
& S, R+S \text { short, } \\
& \epsilon= \pm 1, \quad \eta= \pm 1 \text {, } \\
& \delta= \pm 1 \text {. }
\end{aligned}
$$

The relations (A). Let $Y_{R} / A=X_{R}$. For $R$ short, we claim $Y_{R}$ is abelian. We may assume $R=\left\{r_{1}+\cdots+r_{m+1}, r_{m}+\cdots+r_{n}\right\}$. Then $\left[X_{Q}, X_{S}\right]=X_{R}$ by 
(iv), where $Q=\left\{r_{1}+\cdots+r_{m}, r_{m+1}+\cdots+r_{n}, r_{1}+\cdots+r_{n}\right\}, S=S_{m}$. Since elements of $Y_{R}$ commute with $Y_{Q}, Y_{S}$ modulo $A, Y_{R}$ commutes with $\left[Y_{Q}, Y_{S}\right.$ ]; hence $Y_{R}$ is abelian. Since $b_{R}(\lambda)$ acts nontrivially on $X_{R}$, it also does on $Y_{R}$. So, we define $y_{R}(t)$ as the unique element of $x_{R}(t) \cap\left[Y_{R}, H\right]$.

Regarding the choice of $y_{R}(t)$ for $R$ long, we know that $\left\langle X_{R}, X_{-R}\right\rangle$ is isomorphic to $\mathrm{SU}(3, q)$. By our earlier results, $M_{p}(\mathrm{SU}(3, q))=1$. For $q>2, \mathrm{SU}(3, q)$ is perfect; hence $\left\langle Y_{R}, Y_{-R}\right\rangle=A \times\left\langle Y_{R}, Y_{-R}\right\rangle^{\prime}$, and $\left\langle Y_{R}, Y_{-R}\right\rangle^{\prime} \cong\left\langle X_{R}, X_{-R}\right\rangle$. For $q=2$, $\mathrm{SU}(3,2) / O_{3}(\mathrm{SU}(3,2))=Q_{8}$, the quaternion group. So, for $Y=\left\langle Y_{R}, Y_{-R}\right\rangle, Y / Y^{\prime}$ is an abelian group of order $4|A|$. Assume $R=S_{m}$. Taking $S=S_{m-1}$, we have $\left[X_{R}, b_{S}(\lambda)\right]=X_{R}$ for $\langle\lambda\rangle=K^{\times}$. Then by Fitting's lemma, $Y / Y^{\prime}=\left[Y / Y^{\prime}, H\right] \times$ $\bar{A}\left(\bar{A}=A Y^{\prime} / Y^{\prime}\right)$. Thus, for all $q, Y=Y_{0} \times A$ and $Y_{0}$ is $H$-invariant. Define $y_{R}(t, u)$ as the unique element of $x_{R}(t, u) \cap Y_{0}$.

These $y_{R}(t)$ and $y_{R}(t, u)$ satisfy (C) and (I).

The relations (B). (i) $\left[y_{R}(t), y_{S}(u)\right]=f(t, u)$. This occurs for $n \geq 6$ only. We may assume $R=S_{1}$. Then $S=\left\{r_{1}+\cdots+r_{j}, r_{n-j+1}+\cdots+r_{n}\right\}, 2<j \neq m$, or $\pm\left\{r_{j}+\cdots+r_{k}, r_{n-k+1}+\cdots+r_{n-j+1}\right\}, 2<j<n-k+1, k \neq m$. Suppose the first case holds. Conjugating the relation by $b_{s_{j+1}}(\lambda)$ gives $f(t, u)=f\left(t, \lambda^{-1} u\right)$. Since $f$ is biadditive and $|K| \geq 4, f \equiv 1$ follows. The other cases are handled similarly.

(ii) $\left[y_{R}(t), y_{S}(u)\right]=y_{R+S}(\epsilon t u) f(t, u)$. This occurs for $n \geq 6$ only. We may assume $R=S_{1}$; then $S=\left\{r_{2}+\cdots+r_{k}, r_{n-k+1}+\cdots+r_{n-1}\right\}, m \neq k \neq n$, or $-\left\{r_{1}+\cdots+r_{j}, r_{n-j+1}+\cdots+r_{n}\right\}, j \neq m$. We treat the first case only. If $k=2$, conjugating the relation by $b_{s_{k}}(\lambda)$ gives $f(t, u)=f\left(t, \lambda^{-1} u\right)$. If $k=2$, conjugating by $b_{s_{3}}(\lambda)$ gives $f(t, u)=f\left(t, \lambda^{-1} u\right)$. By (i), $f$ is biadditive and so $f \equiv 1$ as $|K| \geq 4$.

(v) $\left[y_{R}(t, u), y_{S}(v)\right]=f(t, u ; v)$. We may assume $R$ is a root of maximal height $\left\{r_{1}+\cdots+r_{m}, r_{m+1}+\cdots+r_{n}, r_{1}+\cdots+r_{n}\right\}$. Then $S=\left\{r_{j}+\cdots+r_{k}\right.$, $\left.r_{n-k+1}+\cdots+r_{n-j+1}\right\}, j<n-k+1, k<m$, or $-\left\{r_{j}+\cdots+r_{k}, r_{n-k+1}+\cdots\right.$ $\left.+r_{n-j+1}\right\}, k<m, j>1, j<n-k+1$. We treat the first case only. If $k>1$, conjugating by $b_{s_{k}}(\lambda)$ gives $f(t, u ; v)=f\left(t, u ; \lambda^{2} v\right)$. Since $f$ is additive in $v$, $f \equiv 1$ as $|K| \geq 4$. If $j=k=1$, a similar argument works with $b_{s_{2}}(\lambda)$ if $m \geq 3$. Assume $j=k=1, m=2$. For a fixed $v, f$ is a homomorphism of $\left\langle y_{R}(t, u)\right|$ all $t, u\rangle$ into $A$, abelian. So, $f$ depends on $t$ only, and is additive in $t$. Write $f(t, u ; v)=g(t, v)$. Conjugating the relation by $b_{S_{1}}(\lambda), b_{S_{2}}(\mu)$, we get $g(t, v)=$ $g\left(\lambda t, \lambda^{2} v\right),=g\left(\mu \bar{\mu}^{-1} t, \mu^{-1} v\right)$. Taking $\mu=\lambda^{2}, g(t, v)=g\left(\lambda^{3} \bar{\lambda}^{-2} t, v\right)$. For all $q$, there is a $\lambda \in K$ with $\lambda^{3} \bar{\lambda}^{-2} \neq 1$, which gives $g \equiv 1$ with biadditivity.

(iv) $\left[y_{R}(t, u), y_{S}(v, w)\right]=y_{1 / 2(R+S)}(\epsilon t u) f(t, u ; v, w)$. We may take $R=$ $\left\{r_{1}+\cdots+r_{m}, r_{m+1}+\cdots+r_{n}, r_{1}+\cdots+r_{n}\right\}$. Then, $S=-\left\{r_{j}+\cdots+r_{m}, r_{m+1}+\right.$ $\left.\cdots+r_{n-j+1}, r_{j}+\cdots+r_{n-j+2}\right\}, 1<j$. Using (v), $f$ is a bimultiplicative map 
$Y_{R} \times Y_{S} \rightarrow A$. So, $\left[Y_{R}^{\prime}, Y_{S}\right]=1=\left[Y_{R}, Y_{S}^{\prime}\right]$, and $f$ is a function $g(t, v)$ of $t, v$ only, and $g$ is biadditive. If $j<m$, conjugation by $b_{s_{j}}(\lambda)$ gives $g(t, u)=g(t, \lambda u)$; as $|K| \geq 4, g \equiv 1$. If $j=m$, conjugation by $b_{s_{m-1}}(\lambda)$ gives the same result if $m \geq 2$. Assume $m=2, j=2$. Conjugation by $b_{s_{1}}(\lambda)$ gives $g(t, u)=g\left(\lambda t, \lambda^{-1} u\right)$, and, by $b_{S_{2}}(\lambda), g(t, u)=g\left(\lambda \bar{\lambda}^{-1} t, \lambda^{2} \bar{\lambda}^{-1} u\right)$. So $g(t, u)=g\left(\lambda^{3} \bar{\lambda}^{-2} t, u\right)$. Since there is a $\lambda \in K$ with $\lambda^{3} \bar{\lambda}^{-2} \neq 1, g \equiv 1$ by biadditivity.

(vi) $\left[y_{R}(t, u), y_{S}(v)\right]=y_{R+S}(\epsilon \bar{u} v) y_{R+2 S}(\eta t v, \delta u v \bar{v}) f(t, u ; v)$. We may assume $R=\left\{r_{1}+\cdots+r_{m}, r_{m+1}+\cdots+r_{n}, r_{1}+\cdots+r_{n}\right\}$. Then $S=-\left\{r_{1}+\cdots+r_{j}\right.$, $\left.r_{n-j+1}+\cdots+r_{n}\right\}, j<m$. We must take into account $f_{R+S, s}$ of type (iii) which is not yet proven to be trivial. Expanding $\left[y_{R}(t, u), y_{S}\left(v_{1}+v_{2}\right)\right]$ and

$$
\left[y_{R}\left(t_{1}+t_{2}, u_{1}+u_{2}-\gamma \bar{t}_{1} t_{2}\right), y_{S}(v)\right]=\left[y_{R}\left(t_{1}, u_{1}\right) y_{R}\left(t_{2}, u_{2}\right), y_{S}(v)\right]
$$

using the commutator identities and previous relations, we get

(a) $f\left(t, u ; v_{1}+v_{2}\right)=f\left(t, u ; v_{2}\right) f\left(t, u ; v_{1}\right) f_{R+S, s}\left(\epsilon t v_{1}, v_{2}\right)$,

(b) $f\left(t_{1}+t_{2}, u_{1}+u_{2}-\gamma \bar{t}_{1} t_{2} ; v\right)=f\left(t_{1}, u_{1} ; v\right) f\left(t_{2}, u_{2} ; v\right)$.

From (b) we see that $f$ is a homomorphism in the first argument, hence $f(t, u ; v)=$ $g(t, v)$, a function independent of $u$, additive in $t$. Conjugating by $b_{R}(\lambda), b_{S}(\mu)$ gives $g(t, v)=g\left(\lambda^{2} \bar{\lambda}^{-1} t, \lambda^{-1} u\right),=g\left(\mu^{-1} t, \mu^{2} v\right)$, respectively. Taking $\lambda=\mu^{2}$, $g(t, u)=g\left(\mu^{3} \bar{\mu}^{-2} t, u\right)$. There is a $\mu \in K$ with $\mu^{3} \bar{\mu}^{-2} \neq 1$, so additivity in the first argument implies $g \equiv 1$. So, $f \equiv 1$ in (vi). Since $t, v_{1}, v_{2}$ vary independently over $K$, (a) gives $f_{R+S, S} \equiv 1$ in type (iii).

(iii) $\left[y_{R}(t), y_{S}(u)\right]=y_{R+S}(0, \epsilon(t \bar{u}-\bar{t} u)) f(t, u), f \equiv 1$.

This finishes the calculations. In the extension $\hat{G}, V=V_{0} \times A$, where $V_{0}=$ $\left\langle y_{R}(t), y_{S}(u, v) \mid R, S \in \Sigma^{+}, t, u, v \in K\right\rangle$ is isomorphic to $U$, the Sylow p-subgroup of $G$, via $y_{R}() \mapsto x_{R}()$. Since $V$ splits over $A, \hat{G}$ does by Gaschütz's theorem. Hence $m_{p}(G)=1$.

The unitary groups ${ }^{2} A_{3}(q)$. $\Sigma$ has type $A_{3}$ with Dynkin diagram

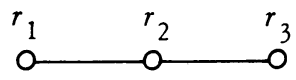

and ${ }^{2} \Sigma$ has type $C_{2}$ with fundamental roots $S_{1}=\left\{r_{1}, r_{2}\right\}$ and $S_{2}=\left\{r_{2}\right\}$. A set of positive roots is $S_{1}, S_{2}, S_{1}+S_{2}, 2 S_{1}+S_{2}$. We carry over notation, etc. from the case ${ }^{2} A_{n}(q), n \geq 5$. The relations of type (B) which occur here are (i), (ii), (v), (vi) only. We deal with $q>2$ only, because ${ }^{2} A_{3} \cong U_{4}(2) \cong \operatorname{PSp}(4,3) \cong B_{2}$ (3) [10], $\left.m_{3^{\prime}}\left(B_{2}(3)\right)=1, m_{2^{\prime}}{ }^{2} A_{3}(2)\right)=1$ and $|Z(S p(4,3))|=2$ implies $m_{2}\left({ }^{2} A_{3}(2)\right)=2$.

The relations (A). For $Y_{R} / A=X_{R}, Y_{R}$ is abelian for $R$ long and the $y_{R}(t)$ 
are chosen as before. Suppose $R=S_{1}$, short and $Y_{R}$ nonabelian. As $x_{R}(t){ }^{b} R^{(\lambda)}=x_{R}\left(\lambda^{2} t\right)$, the eigenvalues for $b_{R}(\lambda)\left(\langle\lambda\rangle=K^{\times}\right)$on the vector space $Y_{R} / A Y_{R^{\prime}}^{\prime}$ are $\lambda^{2 p^{k}}, k=0,1, \cdots, 2 n-1$. Looking at the Lie algebra associated with the $p$-group $Y_{R}, Y_{R}^{\prime}$ is elementary abelian with eigenvalues among $\lambda^{2 p^{i}} \lambda^{2 p^{j}}=$ $\lambda^{2\left(p^{i}+p^{j}\right)}$. Since $Y_{R}^{\prime} \subseteq A$, central, $2 p^{i}+2 p^{j} \equiv 0\left(\bmod q^{2}-1\right)$. We may assume $i=0$ upon multiplying this equation by $p^{-i}$. So, $q^{2}-1=p^{2 n}-1$ divides $2\left(1+p^{j}\right)$. If $q^{2}-1<2\left(1+p^{j}\right), 3>p^{2 n}-p^{j}=p^{2 n-j}\left(p^{j}-1\right)$. If $p^{j}=1, q^{2}-1<2 \cdot 2$ forces $q=2$, the case we excluded. If $p^{j}>1$, then $p^{2 n-j}=2=p^{j}$, so $q=2$ again. Thus, $q^{2}-1=2\left(1+p^{j}\right)$ and $3=p^{j}\left(p^{2 n-j}-2\right)$. Easily, the only possibility is $q=3$. So, for $q \neq 3, Y_{R}$ is abelian and the $y_{R}(t)$ are chosen as before.

Now take $q=3$. Since $x_{R}(t)^{b}{ }^{(\lambda)}=x_{R}\left(\lambda^{2} t\right), b_{R}(\lambda)$ inverts the cyclic group $\left\langle x_{R}(t)\right\rangle$, where $\lambda^{2}=-1$. Choose $y_{R}(t)$ as the unique element of $x_{R}(t) \cap\left[Y_{R}(t)\right.$, $\left.b_{R}(\lambda)\right]$, where $Y_{R}(t) / A=\left\langle x_{R}(t)\right\rangle_{\circ}$ These choices satisfy $y_{R}(m t)=y_{R}(t)^{m}, m$ an integer. Define the factor set $d_{R}$ by $y_{R}(t) y_{R}(u)=y_{R}(t+u) d_{R}(t, u)$.

These representatives $y_{R}(t)$ satisfy (C) and, if $q>3$ or $R$ long, (I) as well.

The relations (B). (i) $\left[y_{R}(t), y_{S}(u)\right]=f(t, u)$. Assume $R=S_{2}, S=2 S_{1}+S_{2}$. Conjugating by $b_{S_{1}}(\lambda), f(t, u)=f\left(\lambda^{-1} \bar{\lambda}^{-1} t, \lambda \bar{\lambda} u\right), \lambda \in K^{\times}$, and by $b_{S_{2}}(\mu), f(t, u)=$ $f\left(\mu^{2} t, u\right), \mu \in K_{0}^{\times}$。 If $q>3$, biadditivity implies $f \equiv 1$. The case $q=3$ is handled in $(\mathrm{v})$.

(ii) $\left[y_{R}(t), y_{S}(u)\right]=f(t, u)$. We may take $R=2 S_{1}+S_{2}, S=S_{1}+S_{2}$. Conjugating by $b_{S_{2}}(\lambda), f(t, u)=f(t, \lambda u), \lambda \in K_{0}$. Biadditivity and $q>2$ imply $f \equiv 1$.

(iv) $\left[y_{R}(t), y_{S}(u)\right]=y_{R+S}(\epsilon(t \bar{u}+\bar{t} u)) f(t, u)$. Using (ii), $f$ is biadditive. Assume $R=S_{1}, S=S_{1}+S_{2}$. Conjugating by $b_{S_{1}}(\lambda), f(t, u)=f\left(\lambda^{2} t, \lambda \bar{\lambda}^{-1} u\right)$, and applying $b_{s_{2}}(\mu), f(t, u)=f\left(\mu^{-1} t, \mu u\right), \mu \in K_{0}^{\times}$. Taking $\lambda=\mu, f(t, u)=f\left(\mu^{2} t, u\right)$. If $q>3, f \equiv 1$ follows. If $q=3, f(t, u)=f\left(\lambda^{2} t, \lambda^{-2} u\right)$, for $\lambda \in K^{\times}$, is the best we can do.

(v) $\left[y_{R}(t), y_{S}(u)\right]=y_{R+S}(\epsilon t u) y_{R+2 S}(\eta t u \bar{u}) f(t, u)$. Assume $R=S_{2}, S=S_{1}$. We must take into account $g(v, w)=f_{R_{+}} s, s(v, w)$ of type (iv) and $b(v, w)=$ $f_{R}+2 S, R(v, w)$ of type (i). Expanding $\left[y_{R}\left(t_{1}+t_{2}\right), y_{S}(u)\right]$ and $\left[y_{R}(t), y_{S}\left(u_{1}+u_{2}\right)\right]$ as usual, we get

(a) $f\left(t, u_{1}+u_{2}\right)=f\left(t, u_{1}\right) f\left(t, u_{2}\right) g\left(\epsilon t u_{1}, u_{2}\right) d_{R+S}\left(\epsilon t u_{1}, \epsilon t u_{2}\right)$,

(b) $f\left(t_{1}+t_{2}, u\right)=f\left(t_{1}, u\right) f\left(t_{2}, u\right) b\left(\eta t_{1} u \bar{u}, t_{2}\right) d_{R+s}\left(\epsilon t_{1} u, \epsilon t_{2} u\right)$.

Conjugating the relation by $b_{R+2 S}(\mu)$ gives $f(t, u)=f(t, \mu u), \mu \in K_{0}^{\times}$. If $q>3, f$ is biadditive; taking $\mu \neq 1$ gives $f \equiv 1$.

Now let $q=3$. Recall that $d_{R+S}(v, \pm v)=1$. Taking $u_{1}= \pm u_{2}$ in (a), we get

(c) $f(t,-u)=f(t, u)^{2} g(\epsilon t u, u)$,

(d) $1=f(t, u) f(t,-u) g(\epsilon t u,-u)$. 
Thus, $f(t, u)^{3}=1$. Since $f(t, u)=f(t,-u), f(t, u)^{-1}=g(\epsilon t u, u)$. Taking $t_{1}=t_{2}$ in (b), we get

(e) $f(-t, u)=f(t, u)^{2} f_{R+2 s, s}(\eta t u \bar{u}, t)$.

Hence, $1=f(-t, u)^{-1} f(t, u)^{2} f_{R+2 s} s(\eta t u \bar{u}, t)=g(-\epsilon t u, u) g(\epsilon t u, u)$.

$f_{R+2 S, s}(\eta t u \bar{u}, t)=f_{R+2 S, s}(\eta t u \bar{u}, t)$, giving triviality in type (i). Since $f(t, u) \epsilon$ $\langle g(v, w) \mid v, w \in K\rangle=B \subseteq A$, for all $t, u$, (a) or (b) gives $d_{R+S}(t, u) \in B$ for all $t, u$. Hence the $g(v, w)$ generate $B=A$.

Going back to (iv), set $Y_{T}=\left\langle y_{T}(t) \mid t \in K\right\rangle$ for $T=R, S$ (the short roots in that case). Since $g$ is a biadditive map $\left(Y_{R} / Y_{R}^{\prime}\right) \times\left(Y_{R} / Y_{R}^{\prime}\right) \rightarrow A, A$ has at most four generators and $A$ has exponent 3. We can bound $|A|$ further as follows. The function $g$ satisfies $g(t, u)=g\left(\lambda^{2} t, \lambda^{-2} u\right), \lambda \in K^{\times}$. All these relations are a consequence of the relations in which $\lambda$ is a fixed primitive eighth root of unity in $K^{\times}$, as simple calculations verify. Since the relation is biadditive, it suffices to require it for $t, u$ taking values on a basis $E$ for $K$ over the prime field. If we take $E=\left\{1, \lambda^{2}\right\}$, then we get the relations
(1) $g(1,1)=g\left(\lambda^{2}, \lambda^{-2}\right)$,
(3) $g\left(\lambda^{2}, 1\right)=g\left(\lambda^{4}, \lambda^{-2}\right)$,
(2) $g\left(1, \lambda^{2}\right)=g\left(\lambda^{2}, 1\right)$,
(4) $g\left(\lambda^{2}, \lambda^{2}\right)=g\left(\lambda^{4}, 1\right)$.

But (1) and (4) are equivalent, as are (2) and ( 3 ). Hence, we have only two independent relations. Thus, $A$ has at most $4-2=2$ generators. So, $|A| \leq 9$.

At this point, we quote the results of [11], in which Lindsey constructs projective representations of $U_{4}(3)$, which imply that a perfect extension of $U_{4}(3)$ by $Z_{3} \times Z_{3}$ exists. We conclude $m_{3}\left({ }^{2} A_{3}(3)\right)=|A|=9$. Note that the preimage in $G$ of $X_{R}, R$ short, is the direct product of a cyclic group of order 3 with a nonabelian group of order 27 , exponent 3 .

These arguments determine $M_{p}\left({ }^{2} A_{3}(q)\right)$ for all $q$.

The second orthogonal groups ${ }^{2} D_{n}(q), n \geq 4$. $\Sigma$ denotes a root system of type $D_{n}$. with Dynkin diagram

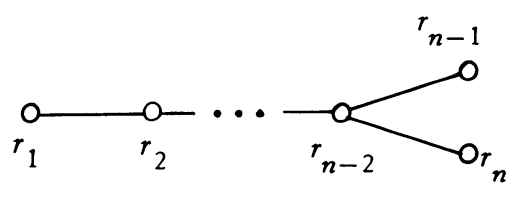

$\Sigma$ is a root system of type $B_{n-1} . S_{1}=\left\{r_{1}\right\}, \cdots, S_{n-2}=\left\{r_{n-2}\right\}$ is a set of long roots, $S_{n-1}=\left\{r_{n-1}, r_{n}\right\}$ a short root, all forming a set of fundamental roots for ${ }^{2} \Sigma$. 
(i) $\left[x_{R}(t), x_{S}(u)\right]=1$

(B) (iii)

$$
\begin{array}{ll}
=x_{R+S}(\epsilon t u) & R, S, R+S \text { long, } \epsilon= \pm 1, \\
=x_{R+S}(\epsilon(t \bar{u}+\overline{t u})) & R, S \text { short, } R+S \text { long, } \\
=1 & R \text { long, } S \text { short, } \\
=x_{R+S}(\epsilon t u)_{R+2 S}(\eta t \bar{t} u) & R \text { long, } S \text { short, } \\
& R+S \text { short, } R+2 S \text { long, } \\
& \epsilon= \pm 1, \quad \eta= \pm 1 .
\end{array}
$$$$
R, S \text { long, }
$$

The relations (A). If $R$ is long, by transitivity of the Weyl group on roots of ${ }^{2} \Sigma$ of the same length, we may assume $R=\left\{r_{1}+r_{2}\right\}$. By (ii), $X_{R}=\left[X_{R_{1}}, X_{R_{2}}\right]$, and by (i) $\left[X_{R}, X_{R_{1}}\right]=\left[X_{R}, X_{R_{2}}\right]=1$. So, if we let $Y / A=\left\langle X_{R_{1}}, X_{R_{2}}\right\rangle$, then the class of $Y$ is 2 or 3. Setting $Y_{R} / A=X_{R}$, we get $\left[Y_{R}, Y_{R}\right] \subseteq\left[A Y^{\prime}, A Y^{\prime}\right]=1$, i.e., $Y_{R}$ is abelian. If $q>2$, then it is easily verified that $H$ acts nontrivially on $X_{R}$, and on $Y_{R}$. We define $y_{R}(t)$ as the unique element of $x_{R}(t) \cap\left[Y_{R}, H\right]$ (use Fitting's lemma). In the case $q=2$, define $y_{R}(1)=\left[x_{R_{1}}(1), x_{R_{2}}(1)\right]$. In fact, we have $y_{R}(1)=\left[y_{S}(1), y_{T}(1)\right]$ for any pair $S, T$ of long roots with $S+T=R$ because the Weyl group of ${ }^{2} \Sigma$ is transitive on such pairs, by Lemma 2. These representatives satisfy (C) and, if $q>2$, (I) also. Since the angle between $R, S$ and $R, T$ must be $60^{\circ}$, we will have (I) for $q=2$ if we define $y_{R}(0)=1$, once we prove $f \equiv 1$ in (i).

For short roots $R$, we put off specifying $y_{R}(t) \in x_{R}(t)$. Choose an arbitrary $y_{R}^{*}(t) \in x_{R}(t)$, all short $R, t \in K$.

The relations (B). (i) $\left[y_{R}(t), y_{S}(u)\right]=f(t, u)$ is biadditive. We may assume $R=S_{1}$ then $S=\left\{r_{i}+\cdots\right\},-\left\{r_{i}+\cdots\right\}, i \geq 3,\left\{r_{1}+r_{2}+\cdots\right\},-\left\{r_{2}+\cdots\right\}$, or $-\left\{r_{1}+2 r_{2}+\cdots\right\}$. In the first, second, and fifth case, $R$ and $S$ are orthogonal. In the third and fourth cases, $R$ and $S$ form a $60^{\circ}$ angle.

We treat the third case, the fourth being similar. Conjugating our relation by a succession of $n_{Q}$, where $W_{Q}$ leaves $R$ invariant, we may assume $S=\left\{r_{1}+r_{2}\right\}$. Choose $v \in K$ with $v+\bar{v}=t$. Then $y_{R}(t) \cdot a=\left[y_{Q}(\epsilon), y_{T}(v)\right]$ (type (iii)), where $a \in A, Q=\left\{r_{1}+\cdots+r_{n-2}+r_{n-1}, r_{1}+\cdots+r_{n-2}+r_{n}\right\}, T=-\left\{r_{2}+\cdots+r_{n-2}+\right.$ $\left.r_{n-1}, r_{2}+\cdots+r_{n-2}+r_{n}\right\}$. Since $\left[Y_{S}, Y_{Q}\right],\left[Y_{S}, Y_{T}\right] \subseteq A$, (type (iv)) $y_{S}(u)$ commutes with $\left[Y_{Q}, Y_{T}\right]$, hence with $y_{R}(t)$. So, $f \equiv 1$ here, and (I) holds for the $y_{R}(t), R$ long, as promised.

Proving $f(t, u)=1$ when $R$ and $S$ are orthogonal is deferred to part (v).

(ii) $\left[y_{R}(t), y_{S}(u)\right]=y_{R+S}(\epsilon t u) f(t, u)$. We may assume $R+S=\left\{r_{1}+2 r_{2}+\cdots\right.$ $\left.+2 r_{n-2}+r_{n-1}+r_{n}\right\}$, a root of maximal height. Then $R=\left\{r_{1}+\cdots+r_{j}\right\}, S=$ 
$\left\{r_{2}+\cdots+r_{j}+2 r_{i+1}+\cdots+2 r_{n-2}+r_{n-1}+r_{n}\right\}, 2 \leq j \leq n-2$, by switching. $R$ and $S$ if necessary。 If $j>2$, conjugation by $b_{S_{2}}(\lambda)$ gives $f(t, u)=f(t, \lambda u)$. Since $R, R+S$ and $S, R+S$ form $60^{\circ}$ angles, the discussion in (i) implies $f$ is biadditive. If $q>2, f \equiv 1$ follows. If $j=2$, conjugating by $b_{s_{2}}(\lambda)$ gives $f(t, u)=f(\lambda t, u)$ and $f \equiv 1$ if $q>2$. If $q=2, f \equiv 1$ by definition.

(iv) $\left[y_{R}(t), y_{S}^{*}(u)\right]=f(t, u)$. We may assume $R=\left\{r_{1}+2 r_{2}+\cdots+2 r_{n-2}+\right.$ $\left.r_{n-1}+r_{n}\right\}$, a root of maximal height. Then $S=\left\{r_{i}+\cdots+r_{n-2}+r_{n-1}, r_{i}+\cdots+\right.$ $\left.r_{n-2}+r_{n}\right\},-\left\{r_{j}+\cdots+r_{n-2}+r_{n-1}, r_{j}+\cdots+r_{n-2}+r_{n}\right\}, j \neq 2$, or $\pm S_{n-1}$. Say $S \neq \pm S_{n-1}$. Then conjugation by $b_{S_{n-1}}(\lambda)$ gives $f(t, u)=f\left(t, \lambda \bar{\lambda}^{-1} u\right)$. Since $K$ has a $\lambda \neq \bar{\lambda}, f \equiv 1$ by biadditivity. The same procedure for $S= \pm S_{n-1}$ gives $f(t, u)=f\left(t, \lambda^{ \pm 2} u\right)$, hence $f \equiv 1$ as $|K| \geq 4$.

(iii) $\left[y_{R}^{*}(t), y_{S}^{*}(u)\right]=y_{R+S}(\epsilon(t \bar{u}+\bar{t} u)) f(t, u)$. We may assume $R=S_{n-1}$; then $S= \pm\left\{r_{1}+\cdots+r_{n-2}+r_{n-1}, r_{i}+\cdots+r_{n-2}+r_{n}\right\}, i \leq n-2$. We assume $S$ positive, the negative case being similar. Using previous relations and the commutator identities, $f(t, u)$ is biadditive. Conjugating by $b_{R}(\lambda)$, we get $f(t, u)=$ $f\left(\lambda^{2} t, \lambda \bar{\lambda}^{-1} u\right)$. If $i<n-2$, conjugation by $b_{R_{i}}(\mu)$ gives $f(t, u)=f(t, \mu u)$; if $i=n-2$, conjugation by $b_{R_{n-3}}(\mu)$ gives $f(t, u)=f\left(t, \mu^{-1} u\right), \mu \in K_{0}$. If $q>2$, choose $\mu \neq 1$ to get $f \equiv 1$. If $q=2, \bar{\lambda}^{-1}=\lambda$, so $f(t, u)=f\left(\lambda^{2} t, \lambda^{2} u\right)$, all $\lambda \in K$. We defer the case $q=2$ until case (v). Note however that $f(t, u)=f(u, t)$ follows from conjugating the arguments by $n_{Q}, Q=\left\{r_{i}+\cdots+r_{n-2}\right\}$. This depends, of course, on choosing a notation for $x_{R}(t)=x_{r_{n-1}}(t) x_{r_{n}}\left(\frac{n}{t}\right)$ and $x_{S}(u)$ in such a way that $x_{R}(t)^{n} Q=x_{S}(t)$ (see Preparation for the Proof).

(v) $\left[y_{R}(t), y_{S}^{*}(u)\right]=y_{R+S}^{*}(\epsilon t u) y_{R+2 S}(\eta t u \bar{u}) f_{R, S}(t, u)$. We may assume $R=S_{1}, S=\left\{r_{2}+\cdots+r_{n-2}+r_{n-1}, r_{2}+\cdots+r_{n-2}+r_{n}\right\}$, or $S=-\left\{r_{1}+\cdots+r_{n-2}+r_{n-1}, r_{1}+\cdots+r_{n-2}+r_{n}\right\}$. We treat only the case $S$ positive. Recall that for $q=2$, certain $f_{Q, T}\left(v_{1}, v_{2}\right)$ are not yet proven to be trivial (types (i), (iii)). Expanding the right side of $\left[y_{R}\left(t_{1}+t_{2}\right), y_{S}^{*}(u)\right]=$ $\left[y_{R}\left(t_{1}\right) y_{R}\left(t_{2}\right), y_{S}^{*}(u)\right]$ by the commutator identity, we get

$$
\begin{aligned}
& y_{R+S}^{*}\left(\epsilon\left(t_{1}+t_{2}\right) u\right) f_{R, S}\left(t_{1}+t_{2}, u\right) \\
& \quad=y_{R+S}^{*}(\epsilon t, u) y_{R+S}^{*}\left(\epsilon t_{2} u\right) f_{R, S}\left(t_{1}, u\right) f_{R, S}\left(t_{2}, u\right) f_{R+2 S, R}\left(\eta t_{1} u \bar{u}, t_{2}\right) .
\end{aligned}
$$

Similarly, expanding $\left[y_{R}(t), y_{S}^{*}\left(u_{1}+u_{2}\right)\right]$ gives

$$
\begin{aligned}
& y_{R+S}^{*}\left(\epsilon t\left(u_{1}+u_{2}\right)\right) f_{R, S}\left(t, u_{1}+u_{2}\right) \\
& \quad=y_{R+S}^{*}\left(\epsilon t u_{2}\right) y_{R+S}^{*}\left(\epsilon t u_{1}\right) f_{R, S}\left(t, u_{2}\right) f_{R, S}\left(t, u_{1}\right) f_{R+S, s}\left(\epsilon t u_{2}, u_{1}\right) .
\end{aligned}
$$


Take $0 \neq u_{1} \neq u_{2} \neq 0$ in $K$. Reversing $u_{1}$ and $u_{2}$ in (b) gives

$$
\begin{aligned}
& y_{R+S}^{*}\left(\epsilon t u_{2}\right) y_{R+S}^{*}\left(\epsilon t u_{1}\right) f_{R, s}\left(t, u_{2}\right) f_{R, s}\left(t, u_{1}\right) f_{R+s, s}\left(\epsilon t u_{2}, u_{1}\right) \\
& \quad=y_{R+s}^{*}\left(\epsilon t u_{1}\right) y_{R+S}^{*}\left(\epsilon t u_{2}\right) f_{R, s}\left(t, u_{1}\right) f_{R, s}\left(t, u_{2}\right) f_{R+s, s}\left(\epsilon t u_{1}, u_{2}\right) .
\end{aligned}
$$

Take $t=\epsilon$. Since the f's are central and $f_{R+s, s}\left(u_{2}, u_{1}\right)=f_{R+s, s}\left(u_{1}, u_{2}\right)$ (see remark at the end of (iii)), cancellation shows that $y_{R+S}^{*}\left(u_{1}\right)$ and $y_{R+S}^{*}\left(u_{2}\right)$ commute, i.e., $Y_{R+S}$ is abelian. So, as usual, we can define $y_{Q}(t)$ as the unique element of $x_{Q}(t) \cap\left[Y_{Q}, H\right]$, for $Q$ short. These $y_{Q}(t)$ satisfy (C) and (I).

Replacing the $y_{R+S}^{*}()$ by the $y_{R+S}()$ in (a) and (b) gives

(c) $f_{R, s}\left(t_{1}+t_{2}, u\right)=f_{R, s}\left(t_{1}, u\right) f_{R, s}\left(t_{2}, u\right) f_{R+2 s, R}\left(\eta t_{1} u \bar{u}, t_{2}\right)$,

(d) $f_{R, s}\left(t, u_{1}+u_{2}\right)=f_{R, s}\left(t, u_{2}\right) f_{R, s}\left(t, u_{1}\right) f_{R+s, s}\left(\epsilon t u_{2}, u_{1}\right)$.

Conjugating by $b_{R+2 S}(\lambda)$ gives $f_{R, s}(t, u)=f_{R, s}(t, \lambda u), \lambda \epsilon_{1} K_{0}$. Now for $q>2$, $f_{R+2 S, R} \equiv 1, f_{R+S, s} \equiv 1$, and $f_{R, S}$ is biadditive; so $f_{R, S} \equiv 1$.

Now, let $q=2$. Then $\epsilon=\eta=1, t=0$ or 1 . Conjugating the original relation by $b_{s}(\lambda)$ shows $f_{R, s}(1, t)=f_{R, S}(1, u)$, all $t, u \in K^{\times}$. Choosing $0 \neq u_{1} \neq u_{2} \neq 0$ from $K$, (d) gives $f_{R, s}(1, u)=f_{R+S, s}\left(u_{2}, u_{1}\right)$, all $u \in K^{\times}$. Since $f_{R+s, s}$ is biadditive, $f_{R, S}(1, u)^{2}=1$. Taking $t_{1}=t_{2}=u=1$ in (c), we get $f_{R+2 S, R}(1,1)=1$. Thus $f \equiv 1$ in type (i). Now, conjugating the original relation by $y_{S_{n-1}}(v)$, we get

$$
\begin{aligned}
& {\left[y_{R}(t), y_{S}(u) y_{S_{n-1}+S}(u \bar{v}+\bar{u} v)\right]} \\
& \quad=y_{R+S}(t u) y_{S_{n-1}+R+S}(t u \bar{v}+t \bar{u} v) f_{R+S, s_{n-1}}(t u, v) y_{R+2 S}(t u \bar{u}) f_{R, s}(t, u) .
\end{aligned}
$$

Expanding the left side, we get $y_{R+s_{n-1}+}(t u \bar{v}+t \bar{u} v)\left[y_{R}(t), y_{S}(u)\right]$, using previous information. Comparison yields $f_{R+} s, s_{n-1}(t u, v)=1$, all $t \in K_{0}, u, v \in K$. Hence $f \equiv 1$ in type (iii). In particular, $f_{R+s, s} \equiv 1$. Using (b), $f_{R, s}(1, u)=$ $f_{R, S}(1, u)^{2}, u \neq 0$, therefore, $f_{R, S} \equiv 1$.

This completes the proof that $m_{p}(\Gamma)=1$.

The groups ${ }^{3} D_{4}(q)$. $\Sigma$ denotes a root system of type $D_{4}$ with Dynkin diagram

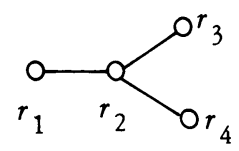

${ }^{3} \Sigma$ is a root system of type $G_{2}$ with a set of fundamental roots $S_{1}=\left\{r_{1}, r_{3}, r_{4}\right\}$ (short), $S_{2}=\left\{r_{2}\right\}$ (long). 


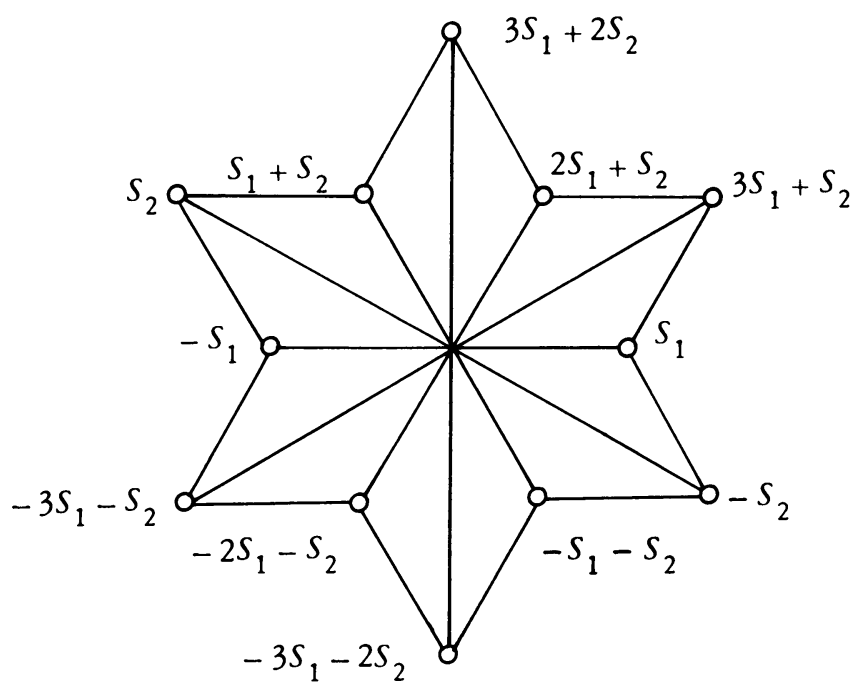

The set of positive roots ${ }^{3} \Sigma^{+}$is

$$
\begin{array}{rlrl}
S_{1} & =\left\{r_{1}, r_{3}, r_{4}\right\}, & 2 S_{1}+S_{2} & =\left\{r_{2}+r_{3}+r_{4}, r_{1}+r_{2}+r_{4}\right. \\
S_{2} & =\left\{r_{2}\right\}, & 3 S_{1}+S_{2} & =\left\{r_{1}+r_{2}+r_{3}+r_{4}\right\}, \\
S_{1}+S_{2} & =\left\{r_{1}+r_{2}, r_{2}+r_{3}, r_{2}+r_{4}\right\}, 3 S_{1}+2 S_{2}=\left\{r_{1}+2 r_{2}+r_{3}+r_{4}\right\} .
\end{array}
$$

In our notation for $x_{s}(t)=x_{s}(t) x_{\bar{s}}(\bar{t}) x_{\bar{s}}=(\overline{\bar{t}}), S$ short, $s$ will be the first root in $S=\{s, \bar{s}, \overline{\bar{s}}\}$ as listed above. Then $x_{s}(t)^{n} R=x_{w(R)}( \pm t)$.
(i) $\left[x_{R}(t), x_{S}(u)\right]=1$
$R, S$ long at a $60^{\circ}$ angle,
(ii)
$=1$
$R$, long, $S$ short at a $30^{\circ}$
(iii)
$=x_{R+S}(\epsilon(t u+\bar{t} \bar{u}+\overline{\bar{t}} \overline{\bar{u}}))$
or $90^{\circ}$ angle,
(iv)

$$
=x_{R+S}(\epsilon t u)
$$
$R, S$ short, $R+S$ long,
$R, S$ at a $60^{\circ}$ angle, $\epsilon= \pm 1$,
$R, S, R+S$ long,
$R, S$ at a $120^{\circ}$ angle, $\epsilon= \pm 1$,

(B)

$$
\begin{aligned}
& =x_{R+S}(\epsilon(\bar{t} \overline{\bar{u}}+\overline{\bar{t}} \bar{u})) x_{2 R+s}(\eta(t \bar{t} \overline{\bar{u}}+\bar{t} \overline{\bar{t}} u+\overline{\bar{t}} t \bar{u})) \\
& \cdot x_{R+2 S}(\delta(t \bar{u} \overline{\bar{u}}+\bar{t} \overline{\bar{u}} u+\overline{\bar{t}} \bar{u}))
\end{aligned}
$$

$$
\begin{aligned}
& R, S \text { short at a } 120^{\circ} \text { angle } \\
& \epsilon= \pm 1, \quad \eta= \pm 1, \quad \delta= \pm 1,
\end{aligned}
$$

(vi)

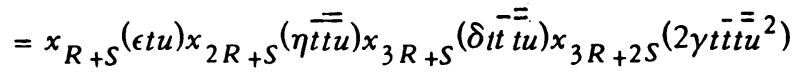

$$
\begin{aligned}
& R \text { short, } S \text { long at a } 150^{\circ} \text { angle, } \\
& \epsilon= \pm 1, \eta= \pm 1, \delta= \pm 1, \gamma= \pm 1 \text {. }
\end{aligned}
$$


The relations (A). Let $V / A=U$ and $Y_{R} / A=X_{R}$. If $R$ is long, assume $R=$ $3 S_{1}+2 S_{2}$; then $X_{R}=Z(U)$. Hence $Y_{R} \subseteq Z_{2}(V)$ and $\left[Y_{R}, Y_{R}\right] \subseteq\left[Y_{R}, A V_{2}\right]=1$; so $Y_{R}$ is abelian. The same argument shows $\left[Y_{R}, Y_{S}\right]=1$, where $S$ is a short root adjacent to $R$. If $S$ (short) and $R$ (long) are orthogonal, then $\left[\left\langle X_{R}, X_{-R}\right\rangle\right.$, $\left.\left\langle X_{S}, X_{-S}\right\rangle\right]=1$ by (ii). But since $\left\langle X_{S}, X_{-S}\right\rangle=\operatorname{SL}\left(2, q^{3}\right)$, it is perfect. Thus, $\left[\left\langle Y_{R}, Y_{-R}\right\rangle,\left\langle Y_{S}, Y_{-S}\right\rangle\right]=1$. Choose temporary representatives $y_{Q}^{*}(t) \in x_{Q}(t)$, $Q \epsilon^{3} \Sigma$. By (v),

$$
\left[y_{Q}^{*}(t), y_{T}^{*}(u)\right]=y_{Q+T}^{*}(\nu) y_{2 Q+T}^{*}\left(v^{\prime}\right) y_{Q+2 T}^{*}\left(v^{\prime \prime}\right) a, \quad a \in A .
$$

The above implies that $y_{Q+T}^{*}\left(v^{\prime \prime \prime}\right)$ commutes with $Y_{2 Q_{+} T}, Y_{Q+2 T}$. Conjugating the left side by $\left.y_{Q+T^{(}}^{*} v^{\prime \prime \prime}\right)$, we get $\left[y_{Q}^{*}(t) y_{2 Q+T^{*}}^{*}\left(t^{\prime}\right), y_{T}^{*}(u) y_{Q+2 T^{*}}^{\left.\left(u^{\prime}\right)\right] \text { which }}\right.$ equals $\left[y_{Q}^{*}(t), y_{T}^{*}(u)\right]$ by the commutator identities and above remarks. Hence, $y_{Q+T}^{*}\left(v^{\prime \prime \prime}\right)$ commutes $w$ ith the right-hand side, i.e., $Y_{S}$ is abelian for $S$ short.

If $R$ is short, or $q>2$ and $R$ is long, the Cartan subgroup acts nontrivially on $Y_{R}$. Define $y_{R}(t)$ as the unique element of $x_{R}(t) \cap\left[Y_{R}, H\right]$. These representatives satisfy (C) and (I). If $R$ is long and $q=2$, choose $\tilde{y}_{S}(t) \in x_{S}(t)$, all long $S$, and define $y_{R}(0)=1$ and $y_{R}(1)=\left[\tilde{y}_{S}(1), \tilde{y}_{T}(1)\right]$, where $S, T$ are long roots with sum $R$. The only ambiguity is the order in which $S, T$ occur. By above remarks, $\tilde{n}_{Q} \in n_{Q} \in X_{Q}, X_{-Q}$ commutes with $Y_{R}$, where $Q$ is a short root orthogonal to $R$; so $y_{R}(1)=y_{R}(1)^{\tilde{n}} Q=\left[y_{T}(1), y_{S}(1)\right]$, proving $y_{R}(1)$ is well defined. This argument also shows $y_{R}(0)=y_{R}(1)^{2}=1$. Hence, all these representatives satisfy (C) and (I).

The relations (B). We have already demonstrated that (i) and (ii) hold for the $y_{R}(t)$.

$$
\text { (iii) }\left[y_{R}(t), y_{S}(u)\right]=y_{R+S}(\epsilon(t u+\bar{t} \bar{u}+\overline{\bar{t}} \overline{\bar{u}})) f(t, u) \text {. }
$$

By (i), $f$ is biadditive. Conjugating by $b_{Q}(\lambda)$, where $Q$ is a long root orthogonal to $S$, we calculate $f(t, u)=f\left(\lambda^{ \pm 1} t, u\right)$. If $q>2$, this gives $f \equiv 1$. The case $q=2$ is treated in (vi).

(iv) $\left[y_{R}(t), y_{S}(u)\right]=y_{R+S}(\epsilon t u) f(t, u)$.

By (i), $f$ is biadditive. Conjugating by $b_{Q}(\lambda)$, where $Q$ is short and orthogonal to $R$, we get $f(t, u)=f\left(t,(\lambda \bar{\lambda} \overline{\bar{\lambda}})^{ \pm 1} u\right)$. So, $f \equiv 1$ if $q>2$. If $q=2, f \equiv 1$ by definition of $y_{R+S}(1)$.

(v) $\left[y_{R}(t), y_{S}(u)\right]=y_{R+S}\left(v_{1}\right) y_{2 R+S}\left(v_{2}\right) y_{R+2 S}\left(v_{3}\right) f(t, u)$, where $v_{1}, v_{2}, v_{3}$ are as stated before. We must take into account the $f_{R+S, S}$ and $f_{R+S, R}$ of type (iii) when $q=2$ here. Expanding

$$
\left[y_{R}(t), y_{S}\left(u_{1}+u_{2}\right)\right]=\left[y_{R}(t), y_{S}\left(u_{2}\right)\right]\left[y_{R}(t), y_{S}\left(u_{1}\right)\right]^{y_{S}\left(u_{2}\right)}
$$


and $\left[y_{R}\left(t_{1}+t_{2}\right), y_{S}(u)\right]$ similarly, we get

(a) $f\left(t, u_{1}+u_{2}\right)=f\left(t, u_{2}\right) f\left(t, u_{1}\right) f_{R+S, s}\left(\epsilon\left(\bar{t} \overline{\bar{u}}_{1}+\overline{\bar{t}} \bar{u}_{1}\right), u_{2}\right)$,

(b) $f\left(t_{1}+t_{2}, u\right)=f\left(t_{1}, u\right) f\left(t_{2}, u\right) f_{R+S, R}\left(\epsilon\left(\bar{t}_{1} \overline{\bar{u}}+\overline{\bar{t}}_{1} \bar{u}\right), t_{2}\right)$.

Let $Q$ be a long root orthogonal to $R$. Then conjugation by $b_{Q}(\lambda)$ gives $f(t, u)=$ $f(t, \lambda u)$. If $q>2$, this with biadditivity of $f$ gives $f \equiv 1$. For $q=2$, let $T$ be the long root $R-S$. Conjugation by $n_{T}$ gives $f(t, u)=f(u, t)$, a fact used in (vi) where this case is settled.

(vi) $\left[y_{R}(t), y_{S}(u)\right]=y_{R+S}(\epsilon t u) y_{2 R+S}(\eta \bar{t} \overline{\bar{t} u}) y_{3 R+}(\delta t \bar{t} \bar{t} u) y_{3 R+2 S}\left(2 y t \bar{t} \bar{t} u^{2}\right) f(t, u)$. Expanding $\left[y_{R}(t), y_{S}\left(u_{1}+u_{2}\right)\right]$, we get, by previous relations,

(c) $f\left(t, u_{1}+u_{2}\right)=f\left(t, u_{2}\right) f\left(t, u_{1}\right)$.

Let $Q$ be a long root orthogonal to $R$. Then, conjugating by $b_{Q}(\lambda), f(t, u)=$ $f\left(t, \lambda^{ \pm 1} u\right)$. If $q>2$, (c) implies $f \equiv 1$. Let $q=2$. Taking $u_{1}=u_{2}=1$, we get $f(t, 1)^{2}=1$. Conjugating by $b_{R}(\lambda)$, we get $f(t, 1)=f\left(t^{\prime}, 1\right)$ for all $t, t^{\prime} \in K^{\times}$. Now, taking the $f$ 's of type (iii) and (v) into account, expanding $\left[y_{R}\left(t_{1}+t_{2}\right)\right.$, $\left.y_{S}(u)\right]$ gives

(d) $f\left(t_{1}+t_{2}, u\right)=f\left(t_{1}, u\right) f\left(t_{2}, u\right) f_{R+S, R}\left(t_{1} u, t_{2}\right) f_{2 R+S, R}\left(t_{1} \bar{t}_{1} u, t_{2}\right) \cdot$ $f_{2 R+S, R+S}\left(t_{1} \bar{t}_{1} u, t_{2} u\right)$.

Now, the last two factors are equal for $u=0,1$, so we cancel them, using (iii). Taking $0 \neq t_{1} \neq t_{2} \neq 0, f(t, u)=f_{R+S, R}\left(t_{1} u, t_{2}\right)$, all $t \in K^{\times}$. Given $t$ and $v$, choose $v_{1}, v_{2} \neq 0, t$ with $v=v_{1}+v_{2}$. Then

$$
f_{R+} S, R(t, v)=f_{R+S, R}\left(t, v_{1}\right) f_{R+S, R}\left(t, v_{2}\right)=f(t, 1)^{2}=1 .
$$

So, the f's of type (v) are identically 1. Now (d) implies, for $t \neq 1, f(t, 1)=$ $f(1,1) f(t-1,1)=f(1,1)^{2}=1$. So, $f \equiv 1$ in (vi). Let $g(t, u)=f_{R+} s, 2 R_{+} s(t, u)$, type (iii). Conjugating both sides of (vi) by $y_{2 R+}(v)$, we get

$$
\begin{aligned}
& {\left[y_{R}(t), y_{S}(u)\right]^{y_{2 R+S}(v)}=\left[y_{R}(t) y_{3 R+S}(t v+\bar{t} \bar{v}+\overline{\bar{t}} \overline{\bar{v}}), y_{S}(u)\right]} \\
& =\left[y_{R}(t), y_{S}(u)\right]_{3 R+2 S}(u(t v+\bar{t} \bar{v}+\overline{\bar{t}} \overline{\bar{v}})) \text {; } \\
& \left\{y_{R+S}(t u)_{2 R+S}(\overline{t \bar{t} u}) y_{3 R+S}(\overline{t \bar{t} t u})\right\}^{y_{2 R}+s^{(v)}} \\
& =y_{R+S}(t u) y_{3 R+2 S}(u(t v+\bar{t} \bar{v}+\bar{t} \overline{\bar{v}})) g(t u, v) .
\end{aligned}
$$

Comparing sides, $g(t u, v)=1$, for all $t, u, v$, finishing off (iii).

The proof of $m_{p}(G)=1$ is complete.

The groups ${ }^{2} E_{6}(q)$. $\Sigma$ denotes a root system of type $E_{6}$, with Dynkin diagram 


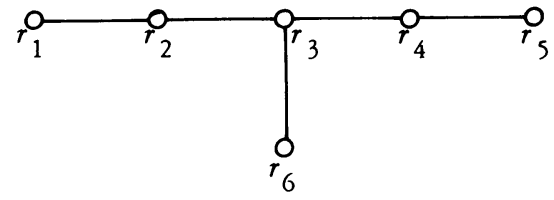

If a positive root $s$ is expressed $\sum_{i=1}^{6} a_{i}{ }_{i}$, then we shall sometimes write

$$
s=\frac{a_{1} a_{2} a_{3} a_{4} a_{5}}{a_{6}} .
$$

${ }^{2} \Sigma$ is a root system of type $F_{4} . S_{1}=\left\{r_{1}, r_{5}\right\}, S_{2}=\left\{r_{2}, r_{4}\right\}$ are short roots and $S_{3}=\left\{r_{3}\right\}, S_{4}=\left\{r_{6}\right\}$ are long roots, forming a set of fundamental roots for ${ }^{2} \Sigma$. We list the positive roots of ${ }^{2} \Sigma$ :

Short roots: $\quad S_{1}=\left\{\begin{array}{cc}10000, & 00001 \\ 0, & 0\end{array}\right\}, \quad S_{1}+2 S_{2}+S_{3}=\left\{\begin{array}{cc}11110, & 01111 \\ 0, & 0\end{array}\right\}$,

$$
\begin{aligned}
& S_{2}=\left\{\begin{array}{cc}
01000, & 00010 \\
0, & 0
\end{array}\right\}, \quad S_{1}+2 S_{2}+S_{3}+S_{4}=\left\{\begin{array}{cc}
11110, & 01111 \\
1, & 1
\end{array}\right\} \text {, } \\
& S_{1}+S_{2}=\left\{\begin{array}{cc}
11000, & 00011 \\
0, & 0
\end{array}\right\}, \quad S_{2}+S_{3}+S_{4}=\left\{\begin{array}{cc}
01100, & 00110 \\
1, & 1
\end{array}\right\} \text {, } \\
& S_{2}+S_{3}=\left\{\begin{array}{cc}
01100, & 00110 \\
0, & 0
\end{array}\right\}, \quad S_{1}+2 S_{2}+2 S_{3}+S_{4}=\left\{\begin{array}{cc}
11210, & 01211 \\
1, & 1
\end{array}\right\} \text {, } \\
& S_{1}+S_{2}+S_{3}=\left\{\begin{array}{cc}
11100, & 00111 \\
0, & 0
\end{array}\right\}, \quad S_{1}+3 S_{2}+2 S_{3}+S_{4}=\left\{\begin{array}{cc}
12210, & 01221 \\
1, & 1
\end{array}\right\} \text {, } \\
& S_{1}+S_{2}+S_{3}+S_{4}=\left\{\begin{array}{cc}
11100, & 00111 \\
1, & 1
\end{array}\right\}, 2 S_{1}+3 S_{2}+2 S_{3}+S_{4}=\left\{\begin{array}{cc}
12211, & 11221 \\
1, & 1
\end{array}\right\}
\end{aligned}
$$

Long roots:

$$
\begin{aligned}
& S_{3}=\left\{\begin{array}{c}
00100 \\
0
\end{array}\right\}, \quad 2 S_{1}+2 S_{2}+S_{3}+S_{4}=\left\{\begin{array}{c}
11111 \\
1
\end{array}\right\} \\
& S_{4}=\left\{\begin{array}{c}
00000 \\
1
\end{array}\right\}, \quad 2 S_{2}+2 S_{3}+S_{4}=\left\{\begin{array}{c}
01210 \\
1
\end{array}\right\} \text {, } \\
& S_{3}+S_{4}=\left\{\begin{array}{c}
00100 \\
1
\end{array}\right\}, \quad 2 S_{1}+2 S_{2}+2 S_{3}+S_{4}=\left\{\begin{array}{c}
11211 \\
1
\end{array}\right\} \text {, } \\
& 2 S_{2}+S_{3}=\left\{\begin{array}{c}
01110 \\
0
\end{array}\right\}, \quad 2 S_{1}+4 S_{2}+2 S_{2}+S_{4}=\left\{\begin{array}{c}
12221 \\
1
\end{array}\right\} \text {, } \\
& 2 S_{2}+S_{3}+S_{4}=\left\{\begin{array}{c}
01110 \\
1
\end{array}\right\}, \quad 2 S_{1}+4 S_{2}+3 S_{3}+S_{4}=\left\{\begin{array}{c}
12321 \\
1
\end{array}\right\} \text {, } \\
& 2 S_{1}+2 S_{2}+S_{3}=\left\{\begin{array}{c}
11111 \\
0
\end{array}\right\}, \quad 2 S_{1}+4 S_{2}+3 S_{3}+2 S_{4}=\left\{\begin{array}{c}
12321 \\
2
\end{array}\right\} \text {. }
\end{aligned}
$$


(i) $\left[x_{R}(t), x_{S}(u)\right]=1$

(ii)

(iii)

(B) (iv)

(vii)

$$
=1
$$$$
=x_{R+S}(\epsilon t u)
$$$$
=x_{R+S}(\epsilon t u)
$$$$
=1
$$$$
=x_{R+S}(\epsilon(t \bar{u}+\overline{t u}))
$$$$
=x_{R+S}(\epsilon t u) x_{R+2 S}(\eta t u \bar{u})
$$

$R, S$ long,

$R, S$ short,

$R, S, R+S$ long, $\epsilon= \pm 1$,

$R, S, R+S$ short, $\epsilon= \pm 1$,

$R$ long, $S$ short,

$R, S$ short, $R+S$ long, $\epsilon= \pm 1$,

$R, R+2 S$ long, $S, R+S$ short, $\epsilon= \pm 1, \quad \eta= \pm 1$.

The relations (A). Let $Y_{R} / A=X_{R}$, any $R \in{ }^{2} \Sigma$. Choose roots $S, T$ with the same length as $R$ with $S+T=R ; S$ and $T$ form a $120^{\circ}$ angle. Also, $\left[x_{S}(t), x_{T}(u)\right]=$ $x_{R}(\epsilon t u)$, and $\left[X_{R}, X_{S}\right]=\left[X_{R}, X_{T}\right]=1$. Hence, $X_{S} X_{T} X_{R}$ is a group of class 2, and its preimage $Y$ in $G$ has class $\leq 3$. Thus, $Y^{\prime}$ is abelian, and so is $Y_{R} \subseteq A Y^{\prime}$ If $R$ is short, $H$ acts nontrivially on $X_{R}$ for all $q$. Using Fitting's lemma, define $y_{R}(t)$ to be the unique element of $x_{R}(t) \cap\left[Y_{R}, H\right]$. The same works for $R$ long unless $q=2$. Let $q=2$. By symmetry under the Weyl group $W$ of ${ }^{2} \Sigma$, we may assume

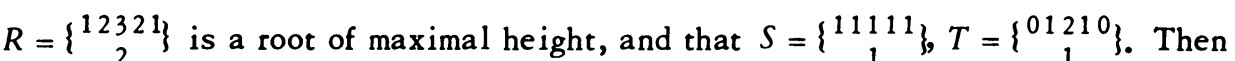
$X_{R} \subseteq Z(U), X_{S}, X_{T} \subseteq U^{\prime}$. So, $Y_{R} \subseteq Z_{2}(V), Y_{S}, Y_{T} \subseteq A V^{\prime} ;$ thus $\left[Y_{R}, Y_{S}\right]=$ $\left[Y_{R}, Y_{T}\right]=1$. Choose any $y_{Q}^{*}(t) \in x_{Q}(t), Q$ long. Define $y_{R}(t)=\left[y_{S}^{*}(t), y_{T}^{*}(1)\right]$, $t=0,1$. By an easy exercise, $W$ is transitive on pairs $S, T$ with $S+T=R$. So, $y_{R}(t)$ is well defined. By the above, $y_{R}(t) y_{R}\left(t^{\prime}\right)=y_{R}\left(t+t^{\prime}\right)$. Thus, in all cases, the $y_{S}(t)$ satisfy $(C)$ and $(\mathrm{I})$.

The relations (B). (i) $\left[y_{R}(t), y_{S}(t)\right]=f(t, u)$. If $R, S$ form a $60^{\circ}$ angle, $f \equiv 1$ by the previous paragraph. If $R, S$ are orthogonal, we mav assume that $R$ is a root of maximal height and that $S$ is positive, $S \neq S_{3}, S_{4}$, by using the Weyl group. As before, $Y_{R} \subseteq Z_{2}(V), Y_{S} \subseteq A V^{\prime}$, so $f \equiv 1$.

(ii) $\left[y_{R}(t), y_{S}(u)\right]=f(t, u)$. In this case, $R$ and $S$ form a $60^{\circ}$ angle. Given $R$, $W$ is transitive on such $S$. So, it is enough to check for one $S$, e.g., $R=S_{1}, S=$ $S_{1}+S_{2}$. Conjugating this relation by $b_{R}(\lambda)$ gives $f(t, u)=f\left(\lambda^{2} t, \lambda^{-1} u\right), \lambda \in K$. Applying $b_{S}(\mu), f(t, u)=f\left(\mu t, \mu^{2} u\right), \mu \in K$. So, $f\left(\lambda^{5} t, u\right)=f(t, u)$. Since $f$ is biadditive, $f \equiv 1$, as $|K| \geq 4$.

(iii) $\left[y_{R}(t), y_{S}(u)\right]=y_{R+S}(\epsilon t u) f(t, u)$. If $q=2, f \equiv 1$ by definition of $y_{R+S}(1)$. Assume $q>2$. By (i) and commutator identities, $f(t, u)$ is biadditive. Using $W$, we may assume $R=S_{3}, S=S_{4}$. Conjugating our relation by $b_{S_{2}}(\lambda)$ we get $f(t, u)=f\left(\lambda^{-1} \bar{\lambda}^{-1} t, u\right)$, all $\lambda \in K$. This, biadditivity and $q>2$ give $f \equiv 1$ as usual. 
(iv) $\left[y_{R}(t), y_{S}(u)\right]=y_{R+S}(\epsilon t u) f(t, u)$. We may assume $R=S_{1}, S=S_{2}$. Using (ii), $f(t, u)$ is biadditive. Conjugating this relation by $b_{R}(\lambda), b_{S}(\mu)$, we get $f(t ; u)=f\left(\lambda^{2} t, \lambda^{-1} u\right)=f\left(\mu^{-1} t, \mu^{2} u\right)$. So, $f(t, u)=f\left(t, \lambda^{3} u\right)$. If $q>2$, biadditivity gives $f \equiv 1$. It turns out that $f$ is not trivial for $q=2$, as we shall see later.

(v) $\left[y_{R}(t), y_{S}(u)\right]=f(t, u)$. $S$ forms either a $60^{\circ}$ or $90^{\circ}$ angle with $R$. We may assume $R$ is a root of maximal height and that $S$ is positive, $S \neq S_{1}, S_{2}$. Arguing as in (i), $f \equiv 1$.

(vi) $\left[y_{R}(t), y_{S}(u)\right]=y_{R+S}(\epsilon(t \bar{u}+\bar{t} u)) f(t, u) . R, S$ are orthogonal short roots. By (v), $f$ is biadditive. We may take $R=S_{1}, S=S_{1}+2 S_{2}+S_{3}$. Conjugating by $b_{R}(\lambda), b_{s_{2}}(\lambda)$, we get $f(t, u)=f\left(\lambda^{2} t, \lambda \bar{\lambda}^{-1} u\right), f(t, u)=f\left(\lambda^{-1} t, \bar{\lambda} u\right)$. So, $f(t, u)=$ $f(t, \lambda \bar{\lambda} u$ ). If $q>2, f \equiv 1$. In (vii), we show $f \equiv 1$ for $q=2$.

(vii) $\left[y_{R}(t), y_{S}(u)\right]=y_{R+S}(\epsilon t u) y_{R+2 S}(\eta t u \bar{u}) f(t, u)$. Expanding $\left[y_{R}\left(t_{1}+t_{2}\right)\right.$, $\left.y_{S}(u)\right]=\left[y_{R}\left(t_{1}\right) y_{R}\left(t_{2}\right), y_{S}(u)\right]$ and $\left[y_{R}(t), y_{S}\left(u_{1}+u_{2}\right)\right]$, we get

(a) $f\left(t_{1}+t_{2}, u\right)=f\left(t_{1}, u\right) f\left(t_{2}, u\right)$,

(b) $f\left(t, u_{1}+u_{2}\right)=f\left(t, u_{2}\right) f\left(t, u_{2}\right) f_{R+s, s}\left(\epsilon t u_{1}, u_{2}\right)$.

By symmetry under $W$, we may assume $R=S_{3}, S=S_{2}$. Conjugating by $b_{S_{1}}(\lambda)$, we get $f(t, u)=f\left(t, \lambda^{-1} u\right.$ ), all $\lambda \in K^{\times}$. For $q>2, f_{R+S, S} \equiv 1$ (type (vi)), which gives $f \equiv 1$. Let $q=2$. Taking $t=1,0 \notin u_{1} \neq u_{2} \neq 0$, we get $f(1,1)=$ $f(1,1)^{2} f_{R+S, S}\left(u_{1}, u_{2}\right)$. Now, $f_{R+S, s}$ biadditive implies $f(1,1)^{2}=1, f(1,1)=$ $f_{R+s, s}\left(u_{1}, u_{2}\right)$. Conjugate the original equation by $y_{s_{2}+s_{3}+s_{4}}(v)$. On the left, we get

$$
\begin{aligned}
& {\left[y_{S_{3}}(t), y_{S_{2}}(u) y_{2 S_{2}+s_{3}+s_{4}}(u \bar{v}+\bar{u} v)\right]=\left[y_{S_{3}}(t), y_{2 S_{2}+s_{3}+s_{4}}(u \bar{v}+\bar{u} v)\right]\left[y_{S_{3}}(t), y_{S_{2}}(u)\right]} \\
& =y_{2 S_{2}+2 s_{3}+s_{4}}(u \bar{v}+\bar{u} v)\left[y_{S_{3}}(t), y_{S_{2}}(u)\right] \text {, }
\end{aligned}
$$

and on the right,

$$
y_{s_{2}+s_{3}}(t u) y_{2 s_{2}+2 s_{3}+s_{4}}(u \bar{v}+\overline{u v}) f_{s_{2}+s_{3}, s_{2}+s_{3}+s_{4}}(t u, v) y_{2 s_{2}+s_{3}}(t u \bar{u}) f(t, u) .
$$

Comparing sides, $f_{S_{2}+s_{3}, S_{2}+s_{3}+S_{4}}(t u, v)=1$, all $t, u, v$, taking care of type (vi). $f \equiv 1$ now follows.

This completes the work for $q>2$. Our next task is to get more detailed information about the $(B, N)$-structure of $\Gamma$ of type ${ }^{2} E_{6}(2)$ and actually construct a nonsplit extension of $\Gamma$ by $Z_{2} \times Z_{2}$. The above calculations show that $M_{2}\left({ }^{2} E_{6}(2)\right)$ is a subgroup of a four group since $A$ is generated by the $f_{R, s}(t, u)$ of type (iv).

The exceptional case ${ }^{2} E_{6}(2)$. 
Lemma. Let $W^{*}$ be the subgroup of $W$ consisting of all $w \in W$ for which, in the expression of $w$ as the product of fundamental reflections, the number of reflections associated with short roots is even. Then $\left|W: W^{*}\right|=2$ and $W^{*}$ is transitive on roots of the same length.

Proof. $W^{*}$ is the kernel of the homomorphism $W \rightarrow Z / 2 Z$ induced by $w_{R} \rightarrow$ $2 Z, R$ long, $w_{S} \rightarrow 1+2 Z, S$ short. So, $\left|W: W^{*}\right|=2$. Since the long roots of ${ }^{2} \Sigma$ form a root system of type $D_{4},\left\langle w_{R}\right| R$ long $\rangle$ is transitive on long roots; hence $W^{*}$ is. The stabilizer in $W$ of a short root $S$ is $W_{0}=\left\langle w_{Q}\right| Q$ orthogonal to $\left.S\right\rangle$, a Weyl group of type $B_{3}$, order $2^{4} \cdot 3$, index $2^{3} \cdot 3$ in $W$. The stabilizer in $W^{*}$ of $S$ is $W_{0} \cap W^{*}=W_{0}^{*}$, which contains some $w_{Q}, Q$ short. So, $W_{0}^{*}$ has index $2^{3} \cdot 3$ in $W^{*}$, and $S$ has $2^{3} \cdot 3$ conjugates under $W^{*}$, which proves transitivity.

We call $w \in W$ even if $w \in W^{*}$, odd if $w \notin W^{*}$.

Lemma. Let $S=\{s, \bar{s}\}$. Every $w \in W_{0}^{*}$ leaves $s$ invariant and every $w \in W \backslash W_{0}^{*}$ switches $s$ and $\bar{s} . W_{0}=W_{0}^{*} \times I_{0}$, where $I_{0}$ is generated by the transformation sending every root orthogonal to $S$ to its negative.

Proof. Suppose $w_{R} \in W_{0}, R=\{r\}$ long. Then $s-c(s, r) r=w_{r}(s)=s$ or $\bar{s}$. If $\bar{s}$, then $r=-c(s, r)^{-1}(\bar{s}-s)$. The Cartan integer $c(s, r)= \pm 1$ since $s \neq \bar{s}$ and all roots of $\Sigma$ have the same length. This forces $r$ to be the sum of two orthogonal roots, impossible.

Since $W_{0}$ contains $W_{1}$, the subgroup leaving $s$ fixed, with index 1 or 2 , we will have $W_{1}=W_{0}^{*}$ if we show $w_{R}$ interchanges $s$ and $\bar{s}$ for $R$ short (all such $w_{R}$ are conjugate in $\left.W_{0}\right)$. We may assume $S=S_{1}, R=S_{1}+2 S_{2}+S_{3}=\{r, \bar{r}\}$. Then

$$
s=\begin{gathered}
10000 \\
0
\end{gathered}, \quad \bar{s}=\begin{gathered}
00001 \\
1
\end{gathered}, \quad r=\begin{gathered}
11110 \\
0
\end{gathered}, \quad \bar{r}=\begin{gathered}
01111 \\
0
\end{gathered},
$$

and

$$
\left(w_{r} w_{r}\right)(s)=w_{r}\left(\begin{array}{c}
11111 \\
0
\end{array}\right)=\begin{gathered}
00001 \\
0
\end{gathered}=\bar{s}
$$

The last part is an exercise.

Now define $x_{s_{1}}(t)=x_{s}(t) x_{\bar{s}}(\bar{t}), s$ as above. By the lemmas, it is well defined to set, for all short roots $S, x_{S}(t)=x_{S_{1}}(t)^{n w}$, where $w \in W^{*}, w\left(S_{1}\right)=S$. Call the unique element of $S$ to which $s$ is conjugate under $W^{*}$ the principal root of $S$. If $w(R)=S$, then $x_{R}(t)^{n_{w}}=x_{S}(t)$ if $w$ is even, $=x_{R}(\bar{t})$ if $w$ is odd.

In $\hat{G}$, we redefine the $y_{R}(t)$ with respect to this new definition of the oneparameter elements of $G$. We also redefine the factor set $f$ by $\left[y_{R}(t), y_{S}(u)\right]=$ $y_{R+S}(\bar{t} \bar{u}) f_{R, S}(t, u)$, where $R, S$ are short roots at a $120^{\circ}$ angle. 
Lemma. (i) $f_{R, S}(t, u)=f_{R, S}(\lambda t, \lambda u)$, all $\lambda \in K^{\times}$.

(ii) $f_{R, s}(t, u)=f_{R, s}(\bar{u}, \bar{t})$.

(iii) $f_{R, S}(t, u)=f_{S, R}(u, t)$.

(iv) $f_{R, S}(t, u)=f_{w(R), w(S)}\left(^{e(w)}, u^{e(w)}\right), e(w)=1,2$ for $w \in W$ even, resp. odd.

Proof. Conjugate the commutator relation defining $f_{R, s}$ by $b_{R}(\lambda)=b_{r}(\lambda) h_{-}(\bar{\lambda})$ to get (i). Setting $\lambda=\bar{t} \bar{u}$ in (i) implies (ii). Since $\left.y_{R+S} \bar{s}^{-} \bar{u}\right)^{2}=1=f(t, u)^{2}$, $\left[y_{R}(t), y_{S}(u)\right]=\left[y_{S}(u), y_{R}(t)\right]$, giving (iii). Part (iv) follows from conjugating the commutator by $n_{w}$, and using $\bar{v}=v^{2}, v \in K$.

We must carefully observe the order of the indices on $f_{R, S^{*}}$. Fix a short root, say $T=2 S_{1}+3 S_{2}+2 S_{3}+S_{4}$. Let $W_{0}$ be the stabilizer of $T$ in $W$. It is easily checked that $W_{0}^{*}$ acts as the full symmetric group on the four pairs $\{R, S\}$ with $R+S=T$ and that $I_{0}$ switches the roots within a pair, and that $R$ is carried to one root only in any pair under $W_{0}^{*}$. Call $S_{1}$ the primary root of the pair $\left\{S_{1}\right.$, $\left.S_{1}+3 S_{2}+2 S_{3}+S_{4}\right\}$ associated with $T$, and call the root of $\{R, S\}$ to which $S_{1}$ is congruent under $W_{0}^{*}$ the primary root of $\{R, S\}$. Primary roots for pairs associated with other short roots are obtained by applying elements of $W^{*}$ to the above situation.

We may now write the factor set without indices as follows. If $R$ is the primary root of $\{R, S\}$, write $f(t, u)=f_{R, S}(t, u)$. If $S$ is primary apply (iv) of the lemma to get $f(\bar{t}, \bar{u})=f_{R, S}(t, u)$. By above remarks, $f$ is independent of $R+S$ or the particular pair $\{R, S\}$ chosen.

Now, consider the following sets of roots

$$
\begin{aligned}
\Sigma_{0}^{+}= & \left\{S_{1}, S_{2}, S_{1}+S_{2}, S_{2}+S_{3}, S_{1}+S_{2}+S_{3}, S_{1}+2 S_{2}+S_{3},\right. \\
& \left.S_{3}, 2 S_{2}+S_{3}, 2 S_{1}+2 S_{2}+S_{3}\right\}, \\
\Sigma_{0}^{-}= & \left\{-R \mid R \in \Sigma_{0}^{+}\right\}, \quad \Sigma_{0}=\Sigma_{0}^{+} \cup \Sigma_{0}^{-}, \\
\Sigma_{1}= & \left\{S_{1}+S_{2}+S_{3}+S_{4}, S_{1}+2 S_{2}+S_{3}+S_{4}, S_{2}+S_{3}+S_{4},\right. \\
& S_{1}+2 S_{2}+2 S_{3}+S_{4}, S_{1}+3 S_{2}+2 S_{3}+S_{4}, 2 S_{1}+3 S_{2}+2 S_{3}+S_{4}, \\
& S_{4}, S_{3}+S_{4}, 2 S_{2}+S_{3}+S_{4}, 2 S_{1}+2 S_{2}+S_{3}+S_{4}, \\
& 2 S_{2}+2 S_{3}+S_{4}, 2 S_{1}+2 S_{2}+2 S_{3}+S_{4}, 2 S_{1}+4 S_{2}+2 S_{3}+S_{4}, \\
& \left.2 S_{1}+4 S_{2}+3 S_{3}+S_{4}, 2 S_{1}+4 S_{2}+3 S_{3}+2 S_{4}\right\} .
\end{aligned}
$$

Then ${ }^{2} \Sigma^{+}$, the positive roots of ${ }^{2} \Sigma$, is the disjoint union of $\Sigma_{0}^{+}$and $\Sigma_{1}$. The parabolic subgroup $P=\left\langle H, X_{R} \mid R \in \Sigma_{1} \cup \Sigma_{0}\right\rangle=C_{G}\left(X_{Q}\right), Q=2 S_{1}+4 S_{2}+3 S_{3}+$ 
$2 S_{4}$ (a root of maximal height) has $M=O_{2}(P)=\left\langle X_{R} \mid R \in \Sigma_{1}\right\rangle$. A complement to $M$ in $P$ is $X_{0}=\left\langle X_{R} \mid R \in \Sigma_{0}\right\rangle$, and a complement to $M$ in $U$ is $U \cap X_{0}=X_{0}^{+}=$ $\left\langle X_{R} \mid R \in \Sigma_{0}^{+}\right\rangle . X_{0}$ is isomorphic to the group $\Gamma$ of type ${ }^{2} A_{5}(2)$, or $\mathrm{SU}(6,2)$, as inspection of the $(B, N)$-structure will show. Note that $M$ is extra special with center $X_{Q}$

We now define an extension of the Borel subgroup $B=U H$ by a four-group which lies in the derived group of the extension. When we show the cohomology class of the extension is stable with respect to $G, M_{2}(G)=Z_{2} \times Z_{2}$ will follow [3, Chapter XII].

Let $1 \rightarrow F \rightarrow G_{0} \rightarrow X_{0} \rightarrow 1$ be the covering of $X_{0}$ by a four-group $F \subseteq G_{0}^{\prime} \cap$ $Z\left(G_{0}\right)$ described in the section on ${ }^{2} A_{5}(2)$. We wish to make $G_{0}$ act via $X_{0}$ on $L=M \times F$ in such a way that $L G_{0}$, with $L \cap G_{0}=F$ has the desired multiplication structure on $V$, the induced extension of $U$. We take $F$ to be the set of all biadditive functions $f(t, u), t, u \in K$, having the same properties as the factor set $f(t, u)$ described previously. Specifically, $f(t, u)=f(\lambda t, \lambda u), \lambda \in K^{\times}, t, u \in K$. Easily, these defining relations imply $F$ is a four-group.

We.regard $G_{0}$ as generated by elements $y_{R}(t)$ which map onto $x_{R}(t) \in X_{0}$ in the above sequence. In the notation of the section on ${ }^{2} A_{5}(2)$, certain $f(t, u)$ generate $F$ and they are involved in the defining commutator relations.

Let $\Delta$ be the set of all pairs $(R, S)$, where $R, S$ are short and form a $120^{\circ}$ angle. Identify $M$ and $F$ with the subgroups $M \times 1$ and $1 \times F$ of $L$. Define the action of $G_{0}$ on $L$ as follows:

$$
\begin{aligned}
& f^{y} s^{(t)}=f \\
& \text { all } f \in F, S \in \Sigma_{0} \text {, all } t \text {, } \\
& x_{R}(t)^{y} S^{(u)}=x_{R}(t)\left[x_{R}(t), x_{S}(u)\right] f\left(t^{a(R)}, u^{a(R)}\right)^{e(R, S)} \\
& \text { all } R \in \Sigma_{1}, S \in \Sigma_{0} \text {, all } t, u \text {, } \\
& e(R, S)=0,(R, S) \notin \Delta, e(R, S)=1,(R, S) \in \Delta, \\
& a(R)=1 \text { if } R \text { is the primary root of }(R, S) \in \Delta \text {, } \\
& a(R)=2 \text { if } S \text { is the primary root of }(R, S) \in \Delta \text {. }
\end{aligned}
$$

The group of automorphisms generated by the action of the $y_{S}(u)$ clearly induces $\bar{X}_{0}=X_{0} / Z\left(X_{0}\right)$ on $L / F \cong M$. We must show that they induce $\bar{X}_{0}$ on $M$. To do so, we must show that the automorphisms $y_{S}(u)^{\prime}$ induced by the $y_{S}(u)$ satisfy the relations of type (A) and (B) which define $X_{0}$. Once this is done, $Z\left(X_{0}\right)$, of order 3 , is seen to act trivially on $L$, as it is trivial on the 2-groups $F$ and $L / F$, by 5.3 .2 of [7].

The relations (A) are clearly satisfied. For (B) we must check that the automorphism $\left[x_{S}(u)^{\prime}, x_{T}(v)^{\prime}\right]$ is the product of the automorphisms $x_{Q}(t)^{\prime} \cdots$ coming 
from the usual expression $\left[x_{S}(u), x_{T}(v)\right]=x_{Q}(t) \cdots$, of type (B), $S, T \in \Sigma_{0}$.

We note that the definition of the action of $y_{S}(u)$ on $x_{R}(t)$ is invariant under the application of any $w \in W_{0}$ to the indexing roots. Hence, it is sufficient to $\operatorname{check}\left[y_{S}(u)^{\prime}, y_{T}(v)^{\prime}\right]$ on each $x_{R}(t)$, all $R \in \Sigma_{1}$, for a representative $\{S, T\}$ from each of the orbits of $W_{0}$ on pairs of distinct roots from $\Sigma_{0}$. These orbits are distinguished by the number of short and long roots in each pair, and the angle between the roots, as discussed in the section on $F_{4}(2)$. We list the orbits, with a representative. We use earlier notation for roots in a system of type $F_{4}$.

$$
\begin{array}{lc}
\left\{11^{\prime} 00,0011^{\prime}\right\} \in\left(l, l, 90^{\circ}\right), & \left\{00 i 1^{\prime}, 0001\right\} \in\left(l, s, 135^{\circ}\right), \\
\left\{11^{\prime} 00,0010\right\} \in\left(l, s, 90^{\circ}\right), & \{0010,0001\} \in\left(s, s, 90^{\circ}\right), \\
\left\{0011^{\prime}, 0010\right\} \in\left(l, s, 45^{\circ}\right), & \left\{0010,11^{\prime} 11\right\} \in\left(s, s, 60^{\circ}\right), \\
& \left\{0010,11^{\prime} 1^{\prime} 1\right\} \in\left(s, s, 120^{\circ}\right) .
\end{array}
$$

The correspondence between root notations is based on

$$
011^{\prime} 0=S_{4}, \quad 0011^{\prime}=S_{3}, \quad 0001=S_{2}, \quad 11^{\prime} 1^{\prime} 1^{\prime}=S_{1} .
$$

For each of the seven pairs, the relations (B) may be checked from Table 5 below, which gives $\left[x_{R}(t), y_{S}(u)\right]$ in row $R$, column $S$, for $R \in \Sigma_{1}, S \in \Sigma_{0}$. The notation $T(v)$ means $x_{T}(v)$ or $y_{T}(v)$. We omit $x_{Q}(t), Q=2 S_{1}+4 S_{2}+3 S_{3}+2 S_{4}$, and elements of $F$, since they are central. We shall also need to know the primary roots for short roots in $\Sigma_{1}$ to keep track of the $f($,$) 's. The se are listed in$ Table 4 and are used to construct Table 5. The details of all these verifications are a multitude of simple calculations left to the reader.

Now, form the semidirect product $L G_{0}$ and factor out the diagonal subgroup in $F \times F$ to get $Q$, our desired extension of $P$. Identify $L, G_{0}$, and $F$ with their images in $Q . Q$ is a proper covering of $P$ as $F \subseteq G_{0}^{\prime} \subseteq Q^{\prime}$. Moreover, $Q$ induces an extension $V$ of $U$ with $V / F \cong U, F \subseteq V^{\prime}$. Our next step is to show that the cohomology class of the extension $E$ of $B$, the Borel subgroup is stable with respect to $G$. This will establish $M_{2}(G)=Z_{2} \times Z_{2}$.

Choose representatives $y(x) \in E$ for each $x \in B$. The factor set $b\left(x, x^{\prime}\right)$ of the extension is defined by $y(x) y\left(x^{\prime}\right)=y\left(x x^{\prime}\right) b\left(x, x^{\prime}\right)$.

We also denote by $b$ the element of the cohomology class of $b$ in $H^{2}(B, F)$. To show $b$ is stable under $G$, we show that the restrictions of $b$ to $U \cap U^{g}$ and $U^{g^{-1}} \cap U$ correspond under the homomorphism of cohomology groups $c_{\boldsymbol{g}}$ : $H^{2}\left(U \cap U^{g}, F\right) \rightarrow H^{2}\left(U^{g} \cap U, F\right)$ whicn are induced by the maps of cocycles $\tilde{c}_{g}(a(x, y))=a\left(x^{g^{-1}}, y^{g^{-1}}\right) \in Z^{2}\left(U^{g^{-1}} \cap U, F\right) \quad$ for $a(x, y) \in Z^{2}\left(U \cap U^{g}, F\right)$. It suffices to let $g$ run over a set of $(B, B)$ double coset representatives. We take 
$\left\{n_{w} \mid w \in W\right\}$ for these representatives. However, by the following result of Glauberman, it suffices to consider only four $n_{w}$, where $w$ runs over a set of fundamental reflections.

Lemma (Glauberman). Let $G$ be a group of Lie type, and let $B, N, n_{w}$ bave their usual meanings. Suppose $\beta$ is a cobomology class of $B$ with

$$
c_{8}\left(\left.\beta\right|_{B \cap B^{8}}\right) \neq\left.\beta\right|_{B^{8}}{ }^{-1} \text {. }
$$

Then (*) bolds for $g=n_{r}, r$ a fundamental root.

Proof. Let $R$ be a maximal intersection for which (*) holds. $R=B \cap B^{g}$, $g=b n u, b \in B, n \in N, u \in U$. Then $R=B \cap B^{b n u}=B \cap B^{n u}$, or $S=R^{u^{-1}}=B \cap$ $B^{n}=B \cap B^{n_{w}}$, some $n_{w} \cdot S$ is also maximal in our sense. Write $n_{w}=n_{1} \cdots n_{k}$ as a product of fundamental reflection $n_{i}$ with $k$ minimal. Then, $s^{n_{k} \cdots{ }^{\cdots} n_{i}} \subseteq U$. If $k \geq 2$, by [18, p. 270], $B \cap B^{n_{k} \cdots n_{j}}<B \cap B^{n_{k} \cdots n_{m}}$, all $m>j$.

Take an intersection $D$ of $B$ with a conjugate of $B$ having $D>S, D^{n_{1}} \subseteq B$.

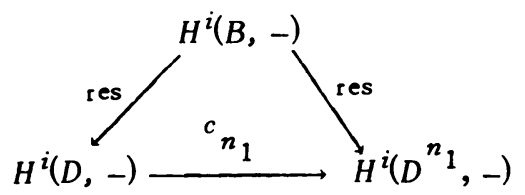

Table 4. Primary Roots for Short Roots in $\Sigma_{1}$

\begin{tabular}{cll}
\hline \hline$Q$ & $(R, S), R$ primary, $R+S=Q$ \\
\hline 1000 & $\left(11^{\prime} 1^{\prime} 1^{\prime}, 1111\right)$ & $\left(111^{\prime} 1,11^{\prime} 11^{\prime}\right)$ \\
& $\left(11^{\prime} 11,111^{\prime} 1^{\prime}\right)$ & $\left(111^{\prime}, 11^{\prime} 1^{\prime} 1\right)$ \\
0100 & $\left(1^{\prime} 11^{\prime} 1^{\prime}, 1111\right)$ & $\left(111^{\prime} 1,1^{\prime} 111^{\prime}\right)$ \\
& $\left(1^{\prime} 111,111^{\prime} 1^{\prime}\right)$ & $\left(1111^{\prime}, 1^{\prime} 11^{\prime} 1\right)$ \\
1111 & $\left(0100,11^{\prime} 11\right)$ & $\left(0001,1111^{\prime}\right)$ \\
& $\left(0010,111^{\prime} 1\right)$ & $\left(1000,1^{\prime} 111\right)$ \\
$1111^{\prime}$ & $\left(11^{\prime} 11^{\prime}, 0100\right)$ & $\left(1^{\prime} 111^{\prime}, 1000\right)$ \\
& $\left(111^{\prime} 1^{\prime}, 0010\right)$ & $\left(1111^{\prime}, 0001^{\prime}\right)$ \\
$111^{\prime} 1$ & $\left(11^{\prime} 1^{\prime} 1,0100\right)$ & $\left(1111,001^{\prime} 0\right)$ \\
& $\left(1^{\prime} 11^{\prime} 1,1000\right)$ & $\left(111^{\prime} 1^{\prime}, 0001\right)$ \\
$111^{\prime} 1^{\prime}$ & $\left(0100,11^{\prime} 1^{\prime} 1^{\prime}\right)$ & $\left(001^{\prime} 0,1111^{\prime}\right)$ \\
& $\left(1000,1^{\prime} 11^{\prime} 1^{\prime}\right)$ & $\left(0001^{\prime}, 111^{\prime} 1\right)$ \\
\hline
\end{tabular}


By maximality of $S$, the image of $\beta$ in $H^{i}\left(D^{n_{1}},-\right)$ along either path is the same. But this, with the commutativity of

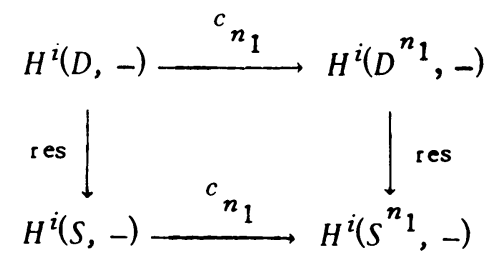

contradicts (*). Therefore, $k=1$, proving the lemma.

Let $n$ be $n_{S_{1}}, n_{S_{2}}$, or $n_{S_{3}}$, and let $b^{\prime}=c_{n}\left(\left.b\right|_{B \cap B^{n}}\right)$ and $b^{\prime \prime}=\left.b\right|_{B \cap B^{n}}$. Then $b^{\prime}$ and $b^{\prime \prime}$. are clearly cohomologous since the cohomology class of $b$, the restriction to $B$ of an extension of $P$, must be stable under $n \in P$.

For any group $J$ and 2-cocycle $j$ which follows, the group determined by $j$ will be $J(j)=\{(x, y) \mid x \in J, y \in F\}$ with multiplication $\left(x_{1}, y_{1}\right)\left(x_{2}, y_{2}\right)=$ $\left(x_{1} x_{2}, y_{1} y_{2} j\left(x_{1}, x_{2}\right)\right)$. In the case $j$ is identically 1 , we regard $J$ as the subgroup $\{(x, 1) \mid x \in J\}$ of $J(j)$. A system $y(x)$ of representatives for $x \in J$ in $J(j)$ are the $(x, 1)$ in this notation.

For $n=n_{w}, w=w_{S_{4}}$, we make specific choices for the $y(x), x \in B$. Let $Q=2 S_{1}+3 S_{2}+3 S_{2}+2 S_{3}+S_{4}$ be the short root of greatest height in ${ }^{2} \Sigma^{+}$.

Let $\Sigma_{2}$ be all roots of ${ }^{2} \Sigma^{+}$orthogonal to $Q$ and let $\Sigma_{3}={ }^{2} \Sigma^{+} \backslash \Sigma_{2}$.

$$
\begin{aligned}
& \Sigma_{2}=\left\{S_{2}, S_{2}+S_{3}, S_{2}+S_{3}+S_{4}, S_{3}, S_{4}, S_{3}+S_{4}\right. \text {, } \\
& \left.2 S_{2}+S_{3}, 2 S_{2}+S_{3}+S_{4}, 2 S_{2}+2 S_{3}+S_{4}\right\} \text {. } \\
& \Sigma_{3}=\left\{S_{1}, S_{1}+S_{2}, S_{1}+S_{2}+S_{3}, S_{1}+S_{2}+S_{3}+S_{4}\right. \text {, } \\
& S_{1}+2 S_{2}+S_{3}, S_{1}+2 S_{2}+S_{3}+S_{4}, S_{1}+2 S_{2}+2 S_{3}+S_{4} \text {, } \\
& s_{1}+3 S_{2}+2 s_{3}+S_{4}, 2 S_{1}+3 S_{2}+2 S_{3}+S_{4} \text {, } \\
& 2 S_{1}+2 S_{2}+S_{3}, 2 S_{1}+2 S_{2}+S_{3}+S_{4}, 2 S_{1}+2 S_{2}+2 S_{3}+S_{4} \text {, } \\
& \left.2 S_{1}+4 S_{2}+2 S_{3}+S_{4}, 2 S_{1}+4 S_{2}+3 S_{3}+S_{4}, 2 S_{1}+4 S_{2}+3 S_{3}+2 S_{4}\right\} \text {. }
\end{aligned}
$$

Reorder the roots of ${ }^{2} \Sigma^{+}=\Sigma_{2} \cup \Sigma_{3}$ as follows:

$$
\begin{aligned}
R \ll S \text { if } & R \in \Sigma_{3}, S \in \Sigma_{2}, \\
& R<S, R, S \in \Sigma_{2}, \\
& R \text { long, } S \text { short, } R, S \in \Sigma_{3}, \\
& R<S, R, S \in \Sigma_{3}, R, S \text { of the same length. }
\end{aligned}
$$

Since we know that in $G_{0}, S_{R}=\left\langle y_{R}(t), y_{-R}(t)\right|$ all $\left.t\right\rangle \cong\left\langle X_{R}, X_{-R}\right\rangle \subseteq X_{0}$, 
R. L. GRIESS, JR.

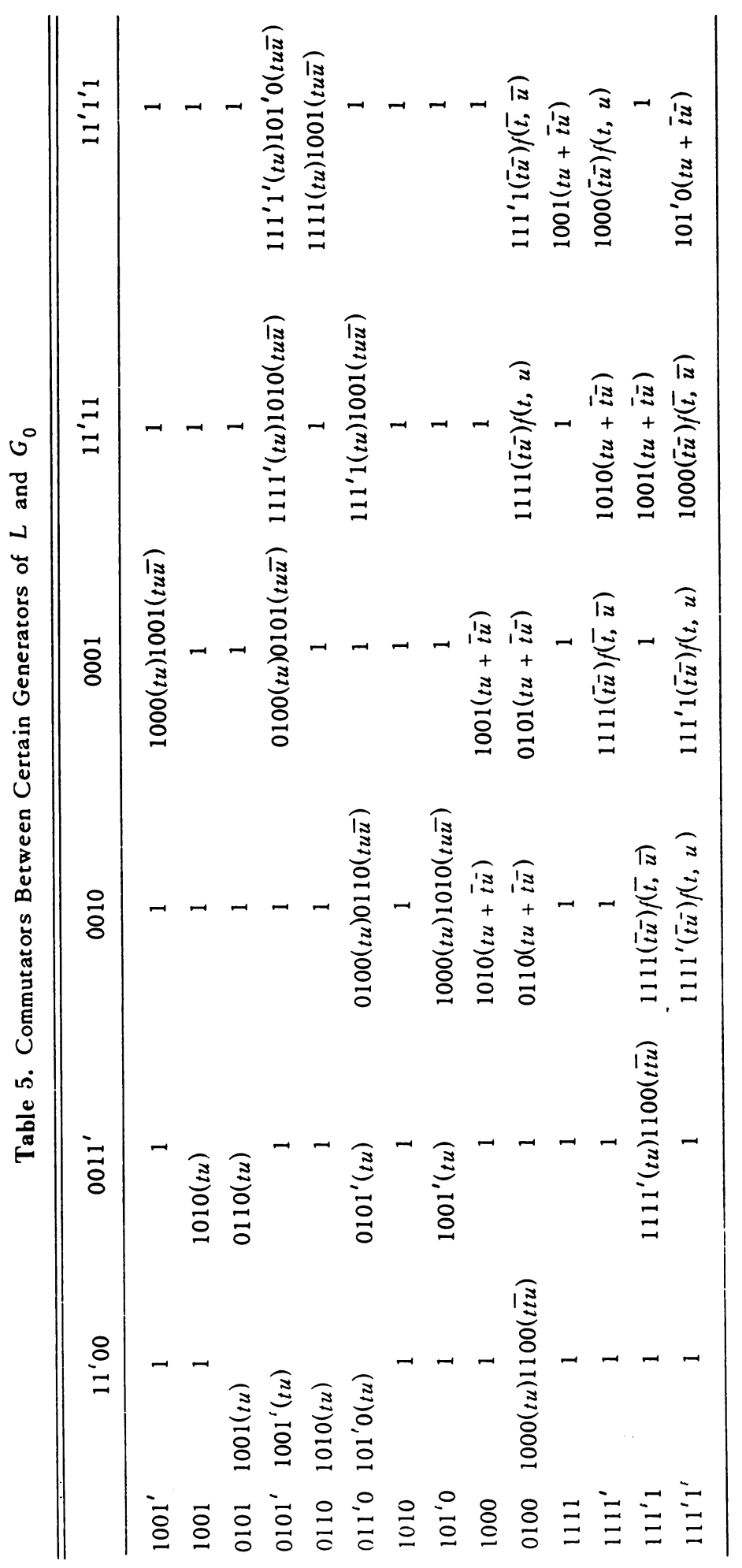


define $k_{R}(\lambda)$ as the unique element of $S_{R}$ mapping onto $b_{R}(\lambda)$. Then $H^{*}=$ $\left\{k_{R}(\lambda) \mid\right.$ all $R$, all $\left.\lambda\right\} \cong H$, the Cartan subgroup of $X_{0}$ (and of $\Gamma$ ). Every element of $B$ may be written uniquely as $x b, x \in U, b \in H$.

Choose $y\left(x_{R}(t)\right)=\left(x_{R}(t), 1\right) \in E$ if $r \in \Sigma_{1}, y\left(x_{R}(t)\right)=y_{R}(t) \in E$ if $R \in \Sigma_{0}$, $y(b)=k$ for $b \in H, k \in H^{*}$ as above. Then set $y(x b)=y(x) y(b), x b \in B, x \in U$, $b \in H$, where $x=\prod x_{R}\left(t_{R}\right)$, the product taken in the order $<$, and where $y(x)=$ $\Pi y\left(x_{R}\left(t_{R}\right)\right)$. The factor set $c\left(x, x^{\prime}\right), x, x^{\prime} \in B$ is defined by $y(x) y\left(x^{\prime}\right)=$ $y\left(x x^{\prime}\right) c\left(x, x^{\prime}\right)$.

Let $M_{0}=\left\langle X_{R} \mid R \in \Sigma_{3}\right\rangle, Y=\left\langle X_{R} \mid R \in \Sigma_{2}\right\rangle$. Then $M_{0} \triangleleft B, M_{0} Y=U, M_{0} \cap$ $Y=1$. By checking the roots involved, we see that $c$ restricted to $Y H$ is identically 1. Also, $c(x, b)=1, x \in U, b \in H, c\left(x, x^{\prime}\right)=1, x \in M_{0}, x^{\prime} \in Y$.

For $x \in U$, write $\operatorname{supp}(x)=\left\{R \in{ }^{2} \Sigma^{+} \mid t_{R} \neq 0\right.$ in $\left.x=\Pi x_{R}\left(t_{R}\right)\right\}$. We may write $x=x_{1} x_{2}$, any $x \in M_{0}$, where $\operatorname{supp}\left(x_{1}\right), \operatorname{supp}\left(x_{2}\right)$ consists of only long, resp.short, roots. Note that if $\operatorname{supp}(x), x \in M_{0}$, consists of long roots only, then $x x^{\prime}=x^{\prime} x$, $c\left(x, x^{\prime}\right)=1=c\left(x^{\prime}, x\right)$, for any $x^{\prime} \in M_{0}$.

For $x=x_{1} x_{2} \in M_{0}, x^{\prime}=x_{1}^{\prime} x_{2}^{\prime} \in M_{0}$ written as above, we use the standard factor set identities (see the section on $F_{4}(2)$ ) to get

$$
\begin{aligned}
c\left(x, x^{\prime}\right)= & c\left(x_{1} x_{2}, x^{\prime}\right)=c\left(x_{1}, x_{2} x^{\prime}\right) c\left(x_{1}, x_{2} x^{\prime}\right) c\left(x_{1}, x_{2}\right) \\
= & c\left(x_{2} x^{\prime}\right) \cdot 1 \cdot 1=c\left(x_{2}, x_{1}^{\prime} x_{2}^{\prime}\right)=c\left(x_{2}, x_{1}^{\prime}\right) c\left(x_{2} x_{1}^{\prime}, x_{2}^{\prime}\right) c\left(x_{1}, x_{2}^{\prime}\right) \\
= & c\left(x_{1}^{\prime}, x_{2}\right) c\left(x_{2} x_{1}^{\prime}, x_{2}^{\prime}\right) \cdot 1=1 \cdot c\left(x_{2} x_{1}^{\prime}, x_{2}^{\prime}\right)=c\left(x_{2}, x_{2}^{\prime}\right) c\left(x_{1}^{\prime}, x_{2} x_{2}^{\prime}\right) \\
& \cdot c\left(x_{1}^{\prime}, x_{2}\right)=c\left(x_{2}, x_{2}^{\prime}\right) \cdot 1 \cdot 1=c\left(x_{2}, x_{2}^{\prime}\right) .
\end{aligned}
$$

We can prove $d$ restricted to $M_{0}$ is identically 1 if we show $c^{\prime}\left(x_{2}, x_{2}^{\prime}\right)=c^{\prime \prime}\left(x_{2}, x_{2}^{\prime}\right)$.

We verify this last condition directly. Write $\left(v_{1}, \cdots, v_{9}\right)$ for the element

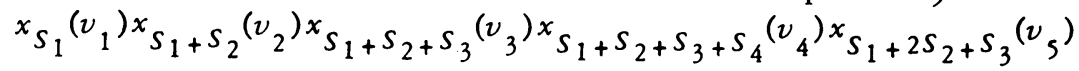

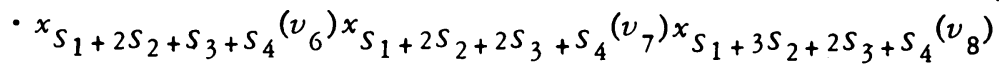

- $x_{2} s_{1}+3 s_{2}+2 s_{3}+s_{4}\left(v_{9}\right)$. A direct computation shows $\left(v_{1}, \cdots, v_{9}\right)^{n}=$ $g\left(v_{1}, v_{2}, v_{4}, v_{3}, v_{6}, v_{7}, v_{8}, v_{9}\right)$, where $\operatorname{supp}(g)$ consists of long roots.

Now take $x_{2}=\left(t_{1}, \cdots, t_{9}\right), x_{2}^{\prime}=\left(u_{1}, \cdots, u_{9}\right)$. Then

$$
\begin{gathered}
y\left(x_{2}\right) y\left(x_{2}^{\prime}\right)=y\left(z \left(t_{1}+u_{1}, t_{2}+u_{2}, t_{3}+u_{3}, t_{4}+u_{4}, t_{5}+u_{5}, t_{6}+u_{6}, t_{7}+u_{7},\right.\right. \\
\left.\left.t_{8}+u_{8}, t_{9}+u_{9}+\bar{t}_{1} \bar{u}_{8}+\bar{t}_{2} \bar{u}_{7}+\bar{t}_{3} \bar{u}_{6}+\bar{t}_{4} \bar{u}_{5}\right)\right) \\
\cdot f\left(t_{1}, u_{8}\right) f\left(\bar{t}_{2}, \bar{u}_{7}\right) f\left(\bar{t}_{3}, \bar{u}_{6}\right) f\left(\bar{t}_{4}, \bar{u}_{5}\right),
\end{gathered}
$$

where $\operatorname{supp}(z)$ contains long roots only. Using the above rule for $x_{2}^{n}, x_{2}^{\prime n}$, we 
calculate

$$
\begin{aligned}
y\left(x_{2}^{n}\right) y\left(x_{2}^{\prime n}\right)= & y\left(z _ { 0 } \left(t_{1}+u_{1}, t_{2}+u_{2}, t_{4}+u_{4}, t_{3}+u_{3}, t_{6}+u_{6}, t_{5}+u_{5}, t_{7}+u_{7},\right.\right. \\
& \left.\left.t_{8}+u_{8}, t_{9}+u_{9}+\bar{t}_{1} \bar{u}_{8}+\bar{t}_{2} \bar{u}_{7}+\bar{t}_{4} \bar{t}_{5}+\bar{t}_{3} \bar{u}_{6}\right)\right) \\
& \cdot f\left(t_{1}, u_{8}\right) f\left(\bar{t}_{2}, \bar{u}_{7}\right) f\left(\bar{t}_{4}, \bar{u}_{5}\right) f\left(\bar{t}_{3}, \bar{u}_{6}\right),
\end{aligned}
$$

where $\operatorname{supp}\left(z_{0}\right)$ contains long roots only. Clearly $c^{\prime}\left(x_{2}, x_{2}^{\prime}\right)=c\left(x_{2}, x_{2}^{\prime}\right)$ and $c^{\prime \prime}\left(x_{2}, x_{2}^{\prime}\right)=c\left(x_{2}^{n}, x_{2}^{\prime n}\right)$ are equal. Thus, $d$ is trivial on $M_{0}$.

Since we have $d$ trivial on $Y H$ and $M_{0},\{(x, 1) \mid x \in H\}=H_{1},\{(x, 1) \mid x \in Y \cap$ $\left.Y^{n}\right\}=Y_{1}$, and $\left\{(x, 1) \mid x \in M_{0}\right\}=M_{1}$ are subgroups of $\left(B \cap B^{n}\right)(d)$. If we can show that all commutators $\left[y\left(x_{R}(t)\right), y\left(x_{S}(u)\right)\right], R \in \Sigma_{3}, S \in \Sigma_{2}$, lie in $M_{1}$ we will get $\left(B \cap B^{n}\right)(d)$ split because $M_{1} Y_{1} H_{1}$ will be a complement to $F$ in $\left(B \cap B^{n}\right)(d)$.

In $\left(B \cap B^{n}\right)\left(b^{\prime}\right)$, we have $\left[y\left(x_{w(R)}(t)\right), y\left(x_{w(S)}(u)\right)\right]=y\left(\left[x_{w(R)}(t), x_{w(S)}(u)\right]\right)$ - $f\left(t^{a(R)}, u^{a(R)}\right)^{e(R, S)}$, because $w$ is even. In $\left(B \cap B^{n}\right)\left(b^{\prime \prime}\right)$, we have $\left[y\left(x_{R}(t)\right)\right.$, $\left.y\left(x_{S}(u)\right)\right]=y\left(\left[x_{R}(t), x_{S}(u)\right]\right) \cdot f\left(t^{a(R)}, u^{a(S)}\right)^{e(R, S)}$. So, in $\left(B \cap B^{n}\right)(d), d=b^{\prime} b^{\prime \prime}$, $\left[y\left(x_{R}(t)\right), y\left(x_{S}(u)\right)\right]=y\left(\left[x_{R}(t), x_{S}(u)\right]\right)$. This verifies the above and completes the proof of stability.

We conclude $M_{2}\left({ }^{2} E_{6}(2)\right) \cong Z_{2} \times Z_{2}$.

\section{CHAPTER III. REE GROUPS}

The groups ${ }^{2} F_{4}(q), q=2^{2 n+1}, n \geq 1$. Let $K=\mathrm{GF}(q), q=2^{2 n+1}$, and let $\theta$ be an automorphism of $K$ with $x^{2 \theta^{2}}=x, x \in K$. We prove $G={ }^{2} F_{4}(q)$ has trivial 2 -part to its multiplier by the usual generator and relations argument for $n \geq 1$, and treat ${ }^{2} F_{4}(2)$ and the simple subgroup ${ }^{2} F_{4}(2)^{\prime}$ separately later.

The following discussion holds even for $q=2$. Notation and $(B, N)$-structure come from Ree [12] and Tits [19], and will be assumed. Define

$$
\begin{aligned}
& u_{1}(t)=a_{1}(t), \quad u_{2}(t)=a_{7}(t), \quad u_{3}(t)=a_{6}(t), \quad u_{4}(t)=a_{10}(t), \\
& u_{5}(t)=a_{5}(t), \quad u_{6}(t)=a_{9}(t), \quad u_{7}(t)=a_{4}(t), \quad u_{8}(t)=a_{3}(t),
\end{aligned}
$$

for all $t \in K$, where the $\alpha_{i}(t)$ are as in [19]. We have

$$
\begin{aligned}
u_{i}(t) u_{i}(u) & =u_{i}(t+u) & & \text { for } i \text { even, } t, u \in K, \\
u_{i}(t) u_{i}(u) & =u_{i}(t+u) u_{i}(v)^{2} & & \text { for } i \text { odd, } t, u \in K, v^{2 \theta+1}=t u^{2 \theta} \\
{\left[u_{i}(t), u_{i}(u)\right] } & =u_{i}(v)^{2} & & \text { for } i \text { odd where } v^{2 \theta+1}=t u^{2 \theta}+t^{2 \theta} u, \\
{\left[u_{i}(t), u_{i}(u)^{2}\right] } & =1 & & \text { for } i \text { odd. }
\end{aligned}
$$

Set $w_{i}=u_{i}(1) u_{i+8}(1) u_{i}(1)$ for $i$ even, $w_{i}=u_{i}(1) u_{i+8}(1)^{2} u_{i}(1)^{-1}$ for $i$ odd. 
Then $w_{i}=w_{i+8}, w_{i}^{2}=1, W=\left\langle w_{i} \mid 1 \leq i \leq 8\right\rangle \cong D_{16}$ and $W$ is the "Weyl group" of the $(B, N)$ pair for $G$ [19]. Abusing terminology slightly, we write $U=$ $\left\langle u_{i}(t) \mid 1 \leq i \leq 8, t \in K\right\rangle, B=U H=N_{G}(U), N=H W\left(=N_{G}(H)\right.$ if $\left.H \neq 1\right), N / H \cong W$ and the extension splits (see [19]). Letting $U_{i}=\left\langle u_{i}(t) \mid t \in K\right\rangle, H$ normalizes each $U_{i}$, and $W$ permutes the $U_{i}$ under conjugation. If we think of the $U_{i}$ as points in Euclidean 2-space (as in the diagram below), the action of $W$ on the "roots" $U_{i}$ may be expressed by interpreting $w_{i}$ as the reflection through the line orthogonal to $U_{i}$

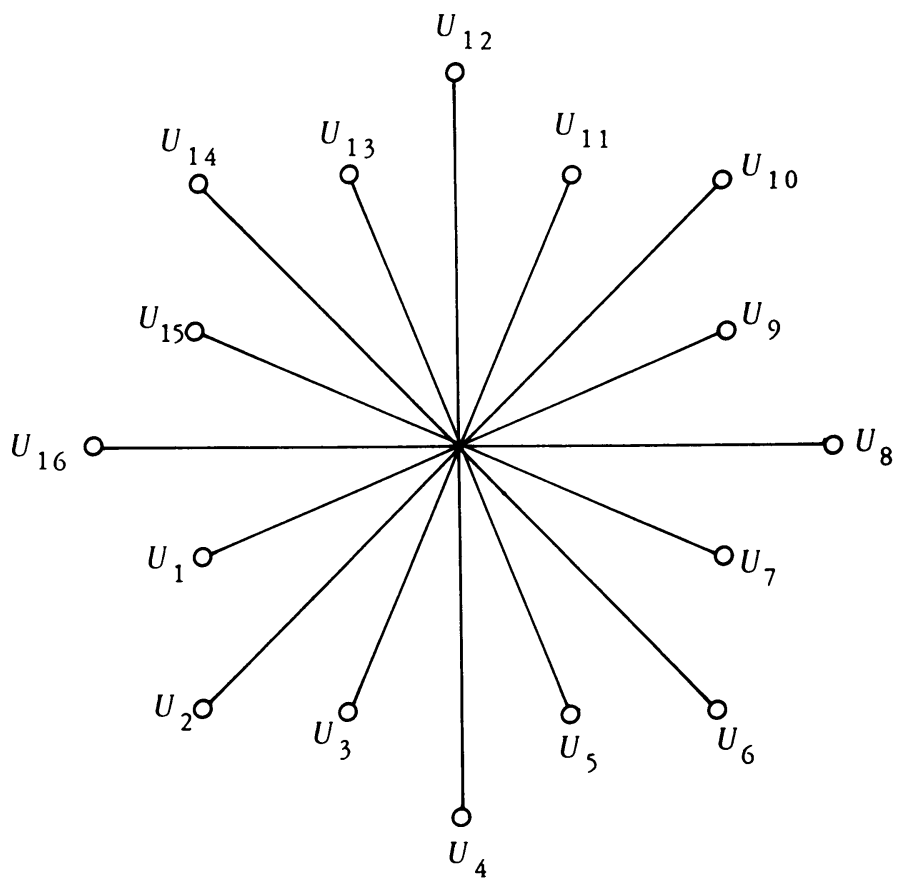

In fact, we have $u_{i}(t)^{w}=u_{j}(t)$ for $w \in W$, where $U_{i}^{w}=U_{j}$. To have some notation for elements of $H$, we momentarily view ${ }^{2} F_{4}(q) \subset F_{4}(q)$, and use the usual Chevalley group notation for elements of $F_{4}(q)$ [4]. For $i$ even, $\lambda \in K^{\times}$define $b_{i}(\lambda) \in{ }^{2} F_{4}(q)$ by $b_{i}(\lambda)=b_{r}\left(\lambda^{2 \theta}\right) b_{r}(\lambda)$, where $r$ is a short root, $\bar{r}$ long, in the expression for $a_{i}(t)=x_{r}\left(t^{\theta}\right) x_{r}(t)$. Similarly, for $i$ odd, define $b_{i}(\lambda)=b_{r}\left(\lambda^{\theta}\right) b_{r}(\lambda)$ where $r, r+\bar{r}$ are short, $\bar{r}$ is long in the expression for $a_{i}(t)=$ $x_{r}\left(t^{\theta}\right) x_{\bar{r}}(t) x_{r+\bar{r}^{-}}\left(t^{\theta+1}\right)$. The action of the $b_{i}(\lambda)$ on the $u_{j}(t)$ is thus calculated in the larger group $F_{4}(q)$. Finally, $\left\langle U_{i}, U_{i+8}\right\rangle \cong{ }^{2} B_{2}(q)$, the Suzuki group $\mathrm{Sz}(q)$, for $i$ odd, and $\cong A_{1}(q) \cong \operatorname{SL}(2, q)$, for $i$ even.

The nontrivial commutator relations among $u_{i}(t), u_{j}(u)$, for $1 \leq i, j \leq 8, i \neq j$ are given below. To get other $\left[u_{i}(t), u_{j}(u)\right], i \neq j+8(\bmod 16)$, conjugate the 
arguments by some $w \in W$ to a pair $u_{i^{\prime}}(t), u_{i^{\prime}}(t)$ for which $1 \leq i^{\prime}, j^{\prime} \leq 8$.

$$
\begin{aligned}
& {\left[u_{1}(t), u_{3}(u)\right]=u_{2}(t u),} \\
& {\left[u_{1}(t), u_{4}(u)\right]=u_{3}(v)^{2}, v^{2 \theta+1}=t u,} \\
& {\left[u_{1}(t), u_{6}(u)\right]=u_{3}(v)^{2} u_{4}\left(t^{2 \theta} u_{u_{5}}\left(v_{0}\right)^{2}, v^{2 \theta+1}=t^{2 \theta+1} u, v_{0}^{2 \theta+1}=t u^{2 \theta},\right.} \\
& {\left[u_{1}(t), u_{7}(u)\right]=u_{2}\left(t^{\theta+1} u_{3} u_{3}\left(t^{2 \theta} u\right) u_{5}\left(t u^{2 \theta}\right)^{3} u_{6}\left(t u^{2 \theta+1}\right),\right.} \\
& {\left[u_{1}(t), u_{8}(u)\right]=u_{2}\left(t ^ { 2 \theta + 2 } u _ { u _ { 3 } } \left(t^{2 \theta+1} u^{2} u_{4}\left(t^{4 \theta+2} u^{2 \theta+1}\right) u_{5}\left(t^{2 \theta+1} u^{2 \theta}\right)^{3}\right.\right.} \\
& \cdot u_{6}\left(t^{2 \theta+2} u^{2 \theta+1}\right) u_{7}(t u) .
\end{aligned}
$$

$\left[u_{2}(t), u_{5}(u)\right]=u_{3}(v)^{2}, v^{2 \theta+1}=t u$,

(B) $\left[u_{2}(t), u_{7}(u)\right]=u_{3}(v)^{2} u_{4}\left(t u^{2 \theta}\right) u_{5}\left(v_{0}\right)^{2}, v^{2 \theta+1}=t^{2 \theta} u, v_{0}^{2 \theta+1}=t u^{2 \theta+1}$,

$\left[u_{2}(t), u_{8}(u)\right]=u_{4}\left(t^{2 \theta} u_{u_{6}}\left(t u^{2 \theta}\right)\right.$,

$\left[u_{3}(t), u_{5}(u)\right]=u_{4}(t u)$,

$\left[u_{3}(t), u_{6}(u)\right]=u_{5}(v)^{2}, \quad v^{2 \theta+1}=t u$,

$\left[u_{3}(t), u_{8}(u)\right]=u_{5}(v)^{2} u_{6}\left(t^{2 \theta} u_{u_{7}}\left(v_{0}\right)^{2}, v^{2 \theta+1}=t^{2 \theta+1} u, v_{0}^{2 \theta+1}=t u^{2 \theta}\right.$,

$\left[u_{4}(t), u_{7}(u)\right]=u_{5}(v)^{2}, \quad v^{2 \theta+1}=t u$,

$\left[u_{5}(t), u_{7}(u)\right]=u_{6}(t u)$,

$\left[u_{5}(t), u_{8}(u)\right]=u_{7}(v)^{2}, \quad v^{2 \theta+1}=t u$.

We now assume $q=2^{2 n+1} \geq 8$. Let $\tilde{G}$ be a central extension of $G$ by a 2group $A$. To establish $m_{2}(G)=1$, we prove any such $\tilde{G}$ splits. By Gaschütz's theorem, it is enough to show that a set of representatives $v_{i}(t)$ for $u_{i}(t)$ in $G$ satisfy (A) and (B), for then $U$ splits, as $U$ is clearly presented by these relations holding among the generators lying in $U$. Note that commutators in $G$ depend only on the coset of $A$ in which the arguments lie. The same holds for conjugating elements. We freely regard elements of $G=\tilde{G} / A$ as cosets of $A$.

For $i$ even, let $v_{i}(t)=\left[u_{i}\left(t\left(1+\lambda^{2}\right)^{-1}\right), b_{i}(\lambda)\right] \in \tilde{G}$. Then these $v_{i}(t)$ satisfy $v_{i}(t) \in u_{i}(t)$ and

$$
\begin{aligned}
& v_{i}(t)^{b}=v_{i}\left(t^{\prime}\right) \text { where } u_{i}(t)^{b}=u_{i}\left(t^{\prime}\right), \text { for } b \in H, \\
& v_{i}(t)^{w}=v_{i^{\prime}}(t) \text { where } u_{i}(t)^{w}=u_{i^{\prime}}(t), \text { for } w \in W .
\end{aligned}
$$

We first proceed to lift the relations holding among the $u_{i}(t), i$ even, to the $v_{i}(t)$.

Set $\left[v_{i}(t), v_{i+2}(u)\right]=f(t, u),\left[v_{i}(t), v_{i+4}(u)\right]=g(t, u)$. Since $f$ and $g$ are $A$ valued, they are bilinear functions of $t$ and $u$. In the first case conjugate by $b_{i+4}(\lambda)$, and in the second, conjugate by $b_{i}(\lambda)$. This yields $f(t, u)=f\left(t, \lambda^{2 \theta} u\right)$. So, $1=f\left(t,\left(1-\lambda^{2 \theta}\right) u\right)$. Since $t$ and $u$ are arbitrary and $q \geq 8, f$ is identically 1 . Also, $g(t, u)=g\left(\lambda^{2} t, u\right)$. Likewise, bilinearity forces $g \equiv 1$. These relations now imply that $f(t, u)$ is bilinear in $\left[v_{i}(t), v_{i+6}(u)\right]=v_{i+2}\left(t^{2 \theta} u\right) v_{i+4}\left(t u^{2 \theta}\right) f(t, u)$, and conjugation 
by $b_{i+4}(\lambda)$ implies $f(t, u)=f\left(t, \lambda^{2 \theta} u\right)$; then $f \equiv 1$, as above.

Working out relations for $v_{i}(t), i$ odd, is a bit more subtle. $Z(U)$ consists of $\left\{u_{5}(t)^{2} \mid t \in K\right\}$ by $[12$, p. 414]. By arguments like the ones in the preceding paragraph, we can easily establish $\left[v_{i}(t), u_{i+1}(u)\right]=1, i$ even, and $\left[u_{i}(t), u_{i+2}(u)\right]=$ $v_{i+1}(t u), i$ odd (the $u_{i}$ 's denote cosets of $A$ ). Inspection of the table of commutator relations shows $u_{3}(t) u_{5}(u) \subseteq A V^{\prime}$ for some $t \neq 0$, all $u \neq 0$. Such elements commute with elements of $\left\{u_{5}(t)^{2} \mid t \in K\right\} \subseteq Z_{2}(V)$, the second center of $V$. Since we already know that for all $t^{\prime}, u^{\prime},\left[u_{3}\left(t^{\prime}\right), u_{5}\left(u^{\prime}\right)^{2}\right]=1$ in $\tilde{G}$, by the above two relations, we get $\left[u_{5}(t), u_{5}(u)^{2}\right]=1$ for all $t, u \in K$. This holds in all $U_{i}$, $i$ odd, by $W$-symmetry.

The subgroup $S=S_{i}=\left\langle U_{i}, U_{i+8}\right\rangle, i$ odd, $G$ is isomorphic to the simple Suzuki group defined over $K$. By Alperin-Gorenstein [1], $m(S)=1$, unless $q=8$. If $\tilde{S}$ denotes the induced extension of $S$ in $\tilde{G}$, we claim $S$ splits. This is clear if $q>8: \tilde{S}=A \times(\tilde{S})^{\prime}$. But if $q=8$ and $\tilde{S}$ does not split, the analysis in [1] shows that in $\tilde{S},\left[u_{i}(t), u_{i}(u)^{2}\right] \neq 1$ for some $t, u$, contrary to the previous paragraph. So, $\tilde{S}=A \times(\tilde{S})^{\prime},(\tilde{S})^{\prime} \cong S$ in all cases. For $i$ odd, we choose $v_{i}(t)$ by the rule $\left\{v_{i}(t)\right\}=u_{i}(t) \cap(\tilde{S})^{\prime}$. Then $\left\langle v_{i}(t) \mid t \in K\right\rangle \cong\left\langle u_{i}(t) \mid t \in K\right\rangle \subseteq G$, and we have the conjugacy properties for these $v_{i}(t)$ under $H$ and $W$ as for $i$ even.

The remaining relations to be lifted to the $v_{i}(t)$ are of the form $\left[u_{i}(t)\right.$, $u_{i+j}(u)$, where $i$ is odd and $3 \leq j \leq 7$. By $W$-symmetry, we may assume $i$, $i+j \in\{1,2, \cdots, 8\}$, and even that $i=1$.

$\left[v_{1}(t), v_{4}(u)\right]=v_{3}(v)^{2} f(t, u), v^{2 \theta+1}=t u$. Using previous relations, $f(t, u)$ is bilinear. Conjugation by, say, $b_{s}(\lambda)$ gives $f \equiv 1$ via the usual argument.

$\left[v_{1}(t), v_{5}(u)\right]=1$ because the commutator lies in $\left[\tilde{s}_{1}, \tilde{s}_{5}\right]$. The latter commutator is trivial because $S_{1}$ and $S_{5}$ are commuting perfect subgroups of $G$.

$$
\begin{aligned}
{\left[v_{1}(t), v_{6}(u)\right] } & =v_{3}(v)^{2} v_{4}\left(t^{2 \theta} u\right) v_{5}(w)^{2} /(t, u), \\
v^{2 \theta+1} & =t^{2 \theta+1} u, \quad w^{2 \theta+1}=t u^{2 \theta} .
\end{aligned}
$$

Using previous relations and taking $u_{1} \neq u_{2}$, we get, by the usual commutator identities, $\left[v_{1}(t), v_{6}\left(u_{1}+u_{2}\right)\right]=\left[v_{1}(t), v_{6}\left(u_{2}\right)\right]\left[v_{1}(t), v_{6}\left(u_{1}\right)\right]$. Comparing each side, we get $f\left(t, u_{1}+u_{2}\right)=f\left(t, u_{2}\right) f\left(t, u_{1}\right)$. Conjugating the original relation by $b_{s}(\lambda)$, we get $f(t, u)=f(t, \lambda u)$, all $t, u \in K, \lambda \in K^{\times}$. So $1=f(t,(1-\lambda) u)$ gives $f \equiv 1$.

$$
\left[v_{1}(t), v_{7}(u)\right]=v_{2}\left(t^{\theta+1} u\right) v_{3}\left(t^{2 \theta} u\right) v_{5}\left(t u^{2 \theta}\right)^{3} v_{6}\left(t u^{2 \theta+1}\right) f(t, u)
$$

Conjugating this relation by $b_{s}(\lambda)$, we get $f(t, u)=f\left(t, \lambda^{-1} \lambda^{2 \theta} u\right)$. Conjugating by $b_{3}(\lambda)$ gives $f(t, u)=f\left(\lambda^{-1} \lambda^{2 \theta} t, u\right)$. These imply $f(t, u)=f\left(t^{\prime}, u^{\prime}\right)$, for all $t, u, t^{\prime}$, 
$u^{\prime} \in K^{\times}$. Now, $\left[v_{1}(t), v_{7}(u)\right]=\left[v_{7}(u), v_{1}(t)\right]^{-1}=\left(\left[v_{1}(u), v_{7}(t)\right]^{w}\right)^{-1}$. So, $f(t, u)=$ $f(u, t)^{-1}=f(t, u)^{-1}$, i.e., $f(t, u)^{2}=1$ for all $t, u \in K^{\times}$. This fact and previous relations allow us to compute that $\left[v_{1}(t), v_{7}(u)^{2}\right]=\left[v_{1}(t), v_{7}(u)\right]\left[v_{1}(t), v_{7}(u)\right]^{v_{7}(u)}=$ $v_{3}\left(t^{2 \theta} u\right)^{2} v_{3}(v)^{2} v_{4}\left(t^{\theta+1} u^{2 \theta+1}\right) v_{5}\left(t u^{2 \theta}\right)^{2} v_{5}\left(v_{0}\right)^{2} v_{6}\left(t u^{2 \theta+1}\right)$ for some $v_{,} v_{0}$ (the point is, $f$ is not present). Also, $v_{7}\left(u^{\prime}\right)^{2}$ commutes with each term on the right, for any $u^{\prime} \in K$. Select $u_{1} \neq u_{2}$ in $K^{\times}$. Then, for some $u_{3}$,

$$
\begin{aligned}
& {\left[v_{1}(t), v_{7}\left(u_{1}+u_{2}\right)\right]=\left[v_{1}(t), v_{7}\left(u_{1}\right) v_{7}\left(u_{2}\right) v_{7}\left(u_{3}\right)^{2}\right]} \\
& \quad=\left[v_{1}(t), v_{7}\left(u_{3}\right)^{2}\right]\left[v_{1}(t), v_{7}\left(u_{2}\right)\right]^{v_{7}\left(u_{3}\right)^{2}}\left[v_{1}(t), v_{7}\left(u_{1}\right)\right]^{v_{7}\left(u_{2}\right) v_{7}\left(u_{3}\right)^{2}} .
\end{aligned}
$$

Using previous relations, we compute both sides and find $f\left(t, u_{1}+u_{2}\right)=$ $f\left(t, u_{1}\right) f\left(t, u_{2}\right)$. Since all these $f$ 's are equal, $f \equiv 1$.

$$
\begin{aligned}
& {\left[v_{1}(t), v_{8}(u)\right]} \\
& \quad=v_{2}\left(t^{2 \theta+2} u\right) v_{3}\left(t^{2 \theta+1} u\right)^{2} v_{4}\left(t^{4 \theta+2} u^{2 \theta+1}\right) v_{5}\left(t^{2 \theta+1}\right)^{3} v_{6}\left(t^{2 \theta+2} u^{2 \theta+1}\right) v_{7}(t u) f(t, u)
\end{aligned}
$$

is the last relation, and we handle it as before. Using previous relations, we calculate $\left[v_{1}(t), v_{8}\left(u_{1}+u_{2}\right)\right]=\left[v_{1}(t), v_{8}\left(u_{2}\right)\right]\left[v_{1}(t), v_{8}\left(u_{1}\right)\right]^{v_{8}\left(u_{2}\right)}$, which gives $f\left(t, u_{1}+u_{2}\right)=f\left(t, u_{1}\right) f\left(t, u_{2}\right)$. Conjugating the original relation by $b_{5}(\lambda)$ gives $f \equiv 1$ as usual.

We have shown that the $v_{i}(t)$ satisfy the relations (A), (B), the requirement for showing $m_{2}(G)=1, n \geq 1$.

The simple Tits group ${ }^{2} F_{4}(2)^{\prime}$ and ${ }^{2} F_{4}(2)$. We now let $G$ be the simple group $F^{\prime}, F={ }^{2} F_{4}(2)$, and prove $m(G)=1$. Since $F / G \cong Z_{2}$, cyclic, this is enough to give $m(F)=1$ as well (11).

We use previous notation as much as possible. Let $u_{i}=u_{i}(1), z=u_{5}^{2}$. Now, $C_{F}(z) \supseteq\left\langle u_{1}, u_{2}, \cdots, u_{9}\right\rangle$. Since the latter group is a maximal parabolic subgroup in $F$, the containment is equality. By the table of commutators $O_{2}\left(C_{F}(z)\right)=$ $\left\langle u_{2}, u_{3}, \cdots, u_{8}\right\rangle,\left\langle u_{1}, u_{3}\right\rangle$ is a Frobenius group of order 20 [19], and $C_{F}(z)=$ $\mathrm{O}_{2}\left(C_{F}(z)\right)\left\langle u_{1}, u_{9}\right\rangle$.

The homomorphism of $F$ onto $Z_{2}$ is induced by sending $u_{i} \rightarrow \overline{0} \in Z / 2 Z$ for $i$ even, $u_{i} \rightarrow \overline{1} \in Z / 2 Z$, for $i$ odd [19]. So $z \in G$, the kernel of this map, and it is easy to determine the elements of $C=C_{G}(z)=G \cap C_{F}(z)$. Letting $T=O_{2}(C)$, $T_{1}=T, T_{i+1}=\left[T_{i}, T\right]$ in the usual lower central series notation, we can easily get 


$$
\begin{aligned}
& T_{1}=\left\langle u_{2}, u_{4}, u_{6}, u_{8}, u_{i} u_{j} \mid i, j \in\{3,5,7\}\right\rangle . \\
& T_{2}=\left\langle u_{4}, u_{6}, u_{3}^{2}, u_{5}^{2}, u_{7}^{2}\right\rangle, \quad T_{3}=\left\langle u_{5}^{2}\right\rangle .
\end{aligned}
$$

$\left|T_{1}\right|=2^{9},\left|T_{2}\right|=2^{5},\left|T_{3}\right|=2$. Let $y$ be any element of order 5 in $C$ (say $y=$ $\left.u_{1}^{-1} u_{9}\right)$. By the multiplication table, $y$ is nontrivial on $X=T_{1} / T_{2}$. Since $|X|=$ $2^{4}, X$ is $y$-irreducible and $X=[X, y]$. Similarly, $T_{2} / T_{3}=\left[T_{2} / T_{3}, y\right]$.

Set $E=\left\langle u_{1} u_{5}, u_{5} u_{9}\right\rangle \subseteq G$. $E$ is a complement in $C$ to $T$, and $E$ is a Frobenius group of order 20 because $\left\langle u_{1}, u_{9}\right\rangle \subseteq F$ is, and $u_{5}$ centralizes $\left\langle u_{1}, u_{9}\right\rangle$. The normalizer in $C$ of the cyclic group of order 5 in $E$ is $\left\langle E, u_{5}^{2}\right\rangle$.

Let $\tilde{G}$ be a central extension of $G$ by a 2 -group $A$. We shall prove that if $A \nRightarrow 1, A \nsubseteq \widetilde{G}^{\prime}$, from which $m_{2}(G)=1$ follows.

As usual, $\tilde{K}$ denotes the induced extension of $K \subseteq G . \tilde{g}$ denotes an arbitrary (unless specified otherwise) representative in $G$ for $g \in G$. We write $\tilde{g} \in g$, regarding $g$ as an $A$-coset in $G$. Since commutators and conjugating elements depend only on their $A$-cosets, elements and subgroups of $G$ act on elements, etc. of $\widetilde{G}$. A word of caution: while elements $u_{1} u_{3}$, etc., lie in $G, u_{1}$ and $u_{3}$ do not. So $u_{1} u_{3}$ is represented in $\tilde{G}$ by $\overbrace{1} u_{3}$, not $\widetilde{u}_{1} \widetilde{u}_{3}$.

Consider $\tilde{C}$ and $S=\widetilde{T}$. The major step is to show $A \cap S^{\prime}=1$. We analyze the $S_{i}$ by the action of $E$ on the $S_{i} / S_{i+1}$.

Since $A$ is central, $T_{4}=1$ implies $S_{5}=1 . \widetilde{T}_{3}$ is clearly abelian since $T_{3}$ is cyclic. We claim $\widetilde{T}_{2}$ is abelian. It suffices to prove that $\tilde{u}, \tilde{v}$ commute, where $u, v \in\left\{u_{3}^{2}, u_{4}, u_{5}^{2}, u_{6}, u_{7}^{2}\right\}$, a set of generators for $T_{2}$.

(a) $u_{5}^{2}$ commutes with the others because $\left[\widetilde{T}_{2}, \widetilde{T_{3}}\right]=\left[A S_{2}, A S_{3}\right] \subseteq S_{5}=1$.

(b) Let $a=\left[u_{u_{3} u_{7}}, u_{7}^{2}\right] \in A$. The commutator is bilinear, so $a^{2}=1$ as $\left(u_{7}^{2}\right)^{2} \in A$. Thus, $1=\left[\left(\widetilde{u_{3} u_{7}}\right)^{2}, \widetilde{u_{7}^{2}}\right]=\left[\widetilde{u_{3}^{2}}, \widetilde{u_{7}^{2}}\right]\left[\widetilde{u_{7}^{2}}, \widetilde{u_{7}^{2}}\right]=\left[\widetilde{u_{3}^{2}}, \widetilde{u_{7}^{2}}\right]$. So, $\widetilde{u}_{3}^{2}$ and $\widetilde{u_{7}^{2}}$ commute.

(c) $\widetilde{u_{4}}$ and $\widetilde{u_{6}}$ commute because $\left[\widetilde{u_{4}}, \widetilde{u_{6}}\right]=\left[\widetilde{u_{4}}, \widetilde{u_{6}}\right]^{u_{10}}=\left[\widetilde{u}_{4} \widetilde{u}_{6} \widetilde{u_{8}}, \widetilde{u_{6}}\right]=$ $\left[\widetilde{u_{4}}, \tilde{u_{6}}\right]\left[\widetilde{u_{8}}, \widetilde{u_{6}}\right]=\left[\widetilde{u_{4}}, \widetilde{u}_{6}\right]^{2}$ by W-conjugation. So, $\left[\widetilde{u_{4}}, \tilde{u}_{6}\right]=1$.

(d) $u_{3}^{2}$ and $\widetilde{u_{4}}$ commute because $\left[\widetilde{u_{3}^{2}}, \widetilde{u_{4}}\right]^{w} 8^{w} 1=\left[\widetilde{u_{5}^{2}}, \widetilde{u_{6}}\right]=1$, by (a).

(e) Let $a=\left[{\widetilde{u_{3} u_{7}}}_{u_{6}}\right]$. As in (b), $a^{2}=1$ and $1=\left[\left(\widetilde{u}_{3} u_{7}\right)^{2}, \widetilde{u}_{6}\right]=$ $\left[\widetilde{u_{3}^{2}}, \widetilde{u}_{6}\right]\left[\widetilde{u_{7}^{2}}, \widetilde{u}_{6}\right]$. Since $\left[\widetilde{u_{7}^{2}}, \widetilde{u}_{6}\right]^{w}{ }^{w}{ }_{8}=\left[\widetilde{u_{5}^{2}}, \widetilde{u}_{4}\right]=1$ by (a) we see that $\tilde{u}_{3}^{2}$ and $\tilde{u}_{6}$ commute.

All other pairs of generators $u, v$ commute because $[u, v]$ is conjugate under $W$ to a pair covered in (a)-(e). So, $\widetilde{T_{2}}$ is abelian. Writing $\widetilde{T_{2}}=\left[\widetilde{T_{2}}, y\right] \times C_{T_{2}}(y)$ by Fitting's lemma, $S_{3}, A \subseteq C{\widetilde{T_{2}}}_{(y)}, S_{3} \cap A=1$ implies that $\widetilde{T_{2}}$ is elementary abelian. In particular, if $g$ is an involution in $G$, then $\tilde{g}$ is an involution as $g$ is conjugate to either $u_{4}$ or $u_{5}^{2}$. 
Assume $A \subseteq S^{\prime}=S_{2}$. Let $L$ be the Lie algebra associated with $S, L=L_{1} \oplus$ $L_{2} \oplus L_{3} \oplus L_{4^{*}} A \subseteq S_{2}$ implies $L_{1} \cong T_{1} / T_{2}$ as $E$-groups. Let $L_{0}=L_{01} \oplus L_{02} \oplus$ .. be the tensor product of $L$ with an algebraically closed field of characteristic 2. Let $\xi_{i}$ be an eigenvector in $L_{01}$ for the eigenvalue $\lambda^{i}$ of $y$ and let $\eta_{i}$ be an eigenvector for $\lambda^{i}$ on $L_{02}$. The $\xi_{i}$ form a basis for $L_{01}$. We may assume that $\xi_{i}^{v}=\xi_{2 i}$ upon altering the $\xi_{i}$ by scalars, where $v \in E, y^{v}=y^{3}$. Similarly on $L_{02}, \eta_{i}^{\nu}=\eta_{2 i}$. Since $L_{03}=\left[L_{01}, L_{02}\right], \xi_{i} \eta_{j}$ with $i+j \equiv 0(\bmod 5)$ are the only nontrivial products among the $\xi_{i} \eta_{j}$ because 1 is the only eigenvalue for $y$ on $L_{03}$. But $\left(\xi_{1} \eta_{4}\right)^{\nu^{e}}=\xi_{2} e \eta_{2}, e=0,1,2,3$ (indices taken mod 5). Thus $\operatorname{dim} L_{03} \leq 1$. The dimension is 1 because $1 \neq u_{5}^{2} \in T_{3}$. Say $\zeta$ generates $L_{03}$. $L_{04}=0$ because $\left[L_{02}, L_{02}\right]=0$ follows from $T_{2}$ abelian, and $\left[L_{01}, L_{03}\right]=0$ follows from $\xi_{i} \zeta$ being an eigenvector for $\lambda^{i} \neq 1$, all $i$, against $L_{04} \subseteq A$ being centralized by $y$. So, $\left|S_{3}\right|=2$.

Our analysis shows that the image of $A \subseteq S_{2}$ in $L$ (resp. $L_{0}$ ) must lie in $L_{2}$ (resp. $L_{02}$ ). Since $L_{01}$ generates $L_{0}$ as an algebra, $A \neq 1$ implies some $\xi_{i} \xi_{j}$, $i+j \equiv 0(\bmod 5)$ must be nonzero. Conjugating this product by $v$ implies all $\xi_{i} \xi_{j}$ with $i+j \equiv 0(\bmod 5)$ are equal and generate the image of $A$. So, $|A| \leq 2$ and $2^{4} \leq\left|S_{2}\right| \leq 2^{5}$.

We now analyze the structure of $C_{G}\left(u_{4}\right)$ and its induced extension in $\widetilde{G}$. Similar results will hold for any $g \in G$ conjugate to $u_{4}$; in particular, the $u_{i}$, $i$ even. $C_{F}\left(u_{4}\right)$ contains $X_{0}=\left\langle u_{1}^{2}, u_{2}, u_{3}, u_{4}, u_{5}, u_{6}, u_{7}^{2}, u_{8}, u_{16}\right\rangle ; X_{0}=$ $O_{2}\left(X_{0}\right)\left\langle u_{8}, u_{16}\right\rangle$ and $\left|X_{0}\right|=2^{10} \cdot 3 . C_{G}\left(u_{4}\right)$ contains $X=G \cap X_{0}=\left\langle u_{1}^{2}, u_{2}, u_{3} u_{5}\right.$ $u_{3}^{2}, u_{4}, u_{6}, u_{7}^{2}, u_{8}, u_{16}$ ) of order $2^{9} \cdot 3$. By the character table of $G$ (M. Hall-J. McKay, unpublished), $X$ is the full centralizer, so the containments are equalities.

In what follows, we shall prove that, for a given $\tilde{u}_{4} \in u_{4}$ in $G$,

(i) precisely one of $\tilde{u}_{4}, \widetilde{u}_{4} a$ is expressible as a commutator in $\tilde{X}$ (equivalently, in a Sylow 2-subgroup of $\tilde{X}$ ); call it $\hat{u}_{4}$,

(ii) $\hat{u}_{4}$ has a square root in $\widetilde{G}$ and $\hat{u}_{4} \cdot \alpha$ does not.

Let $R=O_{2}(X)=\left\langle u_{1}^{2}, u_{2}, u_{3} u_{5}, u_{3}^{2}, u_{5}^{2}, u_{4}, u_{6}, u_{7}^{2}\right\rangle, D=\left\langle u_{8}, u_{16}\right\rangle \cong D_{6}$. $|R|=2^{8},\left|R_{2}\right|=2^{3}, R_{3}=1, R / R_{2}$ and $R_{2}=\left\langle u_{3}^{2}, u_{4}, u_{5}^{2}\right\rangle$ are elementary abelian. The elements $u_{1}^{2}, u_{2}, u_{3} u_{5}, u_{6}, u_{7}^{2}$ generate $R$. The element $r=u_{8} u_{16}$ of order 3 in $D$ acts on $R_{2}$ with indecomposable constituents $R_{21}=\left\langle u_{4}\right\rangle$ and $R_{22}=\left\langle u_{3}^{2}, u_{5}^{2}\right\rangle$. It acts on $\bar{R}=R / R_{2}$ with indecomposable constituents of orders $2,2^{2}, 2^{2}$; namely $E_{1}=\left\langle\bar{u}_{3} u_{5} \bar{u}_{2} \bar{u}_{6}\right\rangle, E_{2}=\left\langle\bar{u}_{2}, \bar{u}_{6}\right\rangle, E_{3}=\left\langle\overline{u_{1}^{2} u_{7}^{2}}, \bar{u}_{2} \bar{u}_{3} \bar{u}_{5} \bar{u}_{6} \overline{u_{7}^{2}}\right\rangle$ and the se are each $D$-invariant .

Our first step is to show $\alpha \notin V_{2}$, where $V=\tilde{R}$. Assume $1 \neq \alpha \in V_{2}$. We argue that $\widetilde{u_{4}}$ remains central in $V$. Since $R \subseteq T=O_{2}\left(C_{G}\left(u_{5}^{2}\right)\right), u_{4} \in T_{2}$, if $\underset{u_{4}}{\text { were }}$ 
noncentral in $V$, then $\left[\widetilde{u}_{4}, x\right]=\alpha$, for some $x \in V$. But this violates $\left[S_{2}, S\right]=S_{3}$, $a \notin S_{3}$. Now, $V_{4}=1$ as $R_{3}=1$. Let $L(V)=L_{1} \oplus L_{2} \oplus L_{3}$ be the Lie algebra associated with $V\left(L_{1}=V / V_{2} \cong R / R_{2}\right.$ here $)$. If $\alpha \in V_{3}=L_{3}$, then $\langle\alpha\rangle=V_{3}$ and $\alpha=\left[x_{1}^{*}, x_{2}^{*}\right]$, where $x_{1} \in V_{1} \backslash V_{2}, x_{2} \in V_{2}\left({ }^{*}\right.$ denotes images in $\left.L(V)\right)$. Since $\tilde{u}_{4}$ is central in $V$, we may take $x_{2} \in \widetilde{R_{22}}$. This means $x_{2} \in u_{3}^{2}, u_{5}^{2}$ or $u_{3}^{2} u_{5}^{2}$, all conjugate in $X$ to $u_{5}^{2}$. Replacing $x_{1}, x_{2}$ by conjugates, we may assume $\left[x_{1}, \widetilde{u_{5}^{2}}\right]=$ $\alpha$, a contradiction to $\left[S_{1}, S_{2}\right]=1$. So, $\alpha \notin V_{3}=L_{3}=1$.

As $a \in V_{2}, a^{*}$ is a nontrivial element of $L_{2}$. Denoting by $L_{0}(V)=L_{01} \oplus$ $L_{02}$ the tensor product of $L(V)$ with an algebraically closed field of characteristic 2, we get $\alpha^{*}=\xi \xi^{\prime}$ in $L(V)$, where $\xi$, $\xi^{\prime}$ are $r$-eigenvectors in $L_{01}$ for eigenvalues $\lambda_{1}, \lambda_{2}$ respectively; $\lambda_{1}^{3}=\lambda_{2}^{3}=1$, and $\lambda_{1} \lambda_{2}=1$ since $\alpha$ is central. $\lambda_{1}=$ $\lambda_{2}=1$ is impossible since the eigenspace in $L_{01}$ for the eigenvalue 1 is onedimensional, generated by the image of $E_{1}$. So, $\lambda_{1} \neq 1 \neq \lambda_{2}, \lambda_{1}^{2}=\lambda_{2}$. Let $\xi_{1}$, $\xi_{3}$ be eigenvectors for $\lambda_{1}$ and let $\xi_{2}, \xi_{4}$ be eigenvectors for $\lambda_{2}$ in $L_{01}$. Then $\xi_{1} \xi_{2}, \xi_{1} \xi_{4}, \xi_{3} \xi_{2}, \xi_{3} \xi_{4}$ span the eigenspace for 1 on $L_{02}$.

We may choose notation so that $\xi_{1}, \xi_{2}$ lie in the subspace of $L_{01}$ generated by $E_{2}$ and $\xi_{3}, \xi_{4}$ lie in the subspace of $L_{01}$ generated by $E_{3}$. Now, we examine commutators involving elements of $V$ corresponding to $E_{2}$ and $E_{3}$. Note that these commutators depend only on the $V_{2}$-cosets of the arguments as $V_{3}=1$.

Since $\left[u_{2}, u_{6}\right]=1$, if $\alpha^{*}=\xi_{1} \xi_{2}$, then $\alpha=\left[\tilde{u}_{2}, \widetilde{u_{6}}\right] \in\left[A S_{2}, A S_{2}\right]=1$, contradiction; so $\xi_{1} \xi_{2}=0$. Now, $\xi_{3} \xi_{4} \neq 0$, because it corresponds to the image in $L_{02}$ of $\widetilde{u_{4}}=\left[u_{1}^{2} u_{7}^{2}, \widetilde{u_{2} u_{3} u_{5}} \widetilde{u_{6}} u_{7}^{2}\right]$.

This leaves $\xi_{1} \xi_{4}$ and $\xi_{2} \xi_{3}$; so we look at $\left[\tilde{e}_{2}, \widetilde{e}_{3}\right], \bar{e}_{2} \in E_{2}, \bar{e}_{3} \in E_{3}$. All these commutators lie in $\widetilde{R_{22}}$. Replacing $e_{2}, e_{3}$ by conjugates, we may assume $\left[\tilde{e}_{2}, \tilde{e}_{3}\right] \in u_{5}^{2} ; e_{1}, e_{2} \in C_{G}\left(u_{5}^{2}\right)$ since $V_{3}=1$. We now list all pairs $\left(\bar{e}_{2}, \bar{e}_{3}\right)$ for which $\left[\tilde{e}_{2}, \widetilde{e}_{3}\right] \in u_{5}^{2}$ :

$$
\left(\bar{u}_{2}, u_{1}^{2} u_{7}^{2}\right), \quad\left(\bar{u}_{6}, \bar{u}_{2} \bar{u}_{3} u_{5} \bar{u}_{6} \bar{u}_{7}^{2}\right), \quad\left(\bar{u}_{2} \bar{u}_{6}, \bar{u}_{1}^{2} \bar{u}_{2} \bar{u}_{3}^{3} u_{5} \bar{u}_{6}\right)
$$

$\left[\tilde{u}_{2}, \widetilde{u_{1}^{2}} \widetilde{u_{7}^{2}}\right]$ is conjugate to $\left[\hat{u}_{2}, \widetilde{u_{6}}, \widetilde{u_{1}^{2}} \widetilde{u_{2} u_{3}^{3} u_{5} \widetilde{u}_{6}}\right]$ in $X$ via $u_{8} \in C_{G}\left(u_{5}^{2}\right)$; hence, they

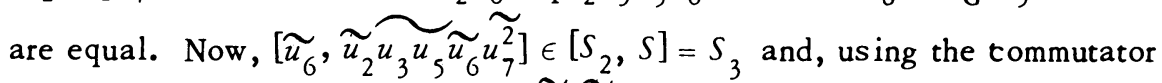
identity and previous results, $\left[\widetilde{u_{2}}, \widetilde{u_{1}^{2}} \widetilde{u_{7}^{2}}\right]=\left[\hat{u}_{2}^{\prime}, u_{7}^{2}\right]$, an element of $\left[S, s_{2}\right]=S_{3}$. Since $\left|u_{5}^{2} \cap S_{3}\right|=1$, all three commutators are equal. The same holds for the commutators $\left[\widetilde{e}_{2}, \widetilde{e}_{3}\right]$ in $u_{3}^{2}$ and in $u_{3}^{2} u_{5}^{2}$. Let $\widehat{u}_{5}^{2}$ denote the unique element of $u_{5}^{2} \cap\left[u_{2}, u_{1}^{2} u_{7}^{2}\right]$, and define $\widehat{u_{3}^{2}}, \widehat{u_{3}^{2} u_{5}^{2}}$ similarly for appropriate $\left[\widetilde{e}_{2}, \widetilde{e}_{3}\right]$. 
We now assert that the three distinct commutators $\left[\widetilde{e}_{2}, \widetilde{e}_{3}\right] \neq 1, \bar{e}_{2} \in E_{2}$, $\bar{e}_{3} \in E_{3}$, together with the identity element, form a subgroup (a four-group). By symmetry under $D$-conjugation, the following argument suffices: for

$$
\widehat{u_{5}^{2}}=\left[\widetilde{u}_{2}, \widehat{u_{1}^{2} u_{7}^{2}}\right], \quad \widehat{u_{3}^{2} u_{5}^{2}}=\left[\widetilde{u}_{2}, \widetilde{u_{2} u_{3}^{3} u_{5}} \widetilde{u_{6}} \widetilde{u_{7}^{2}}\right]
$$

we have

$$
\widehat{u_{5}^{2}} \cdot \widehat{u_{3}^{2} u_{5}^{2}}=\left[\widetilde{u_{2}}, \widetilde{u_{1}^{2} u_{7}^{2}}\right]\left[\widetilde{u}_{2}, \widetilde{u_{2} u_{3}^{3} u_{5} \widetilde{u}_{6} u_{7}^{2}}\right]=\left[\widetilde{u}_{2}, \widetilde{u_{1}^{2} u_{2} u_{3}^{3} u_{5} \widetilde{u}_{6}}\right]=\widehat{u_{3}} .
$$

So, $a$ does not lie in the subgroup of $V_{2}$ generated by the commutators $\left[e_{2}, e_{3}\right]$. This means $\xi_{1} \xi_{4}=\xi_{2} \xi_{3}=0$ and $\alpha \notin V_{2}$. Now, denote by $\widehat{u_{4}}$ the unique element of $u_{4} \cap V_{2}$.

Let $\langle\bar{\alpha}\rangle$ be the image of $\langle\alpha\rangle$ on $V / V_{2} .\langle\bar{\alpha}\rangle$ splits off the fixed point free $\langle r\rangle$ submodules of $V / V_{2}$ by Fitting's lemma. So, if $\alpha \in Y^{\prime}$, where $Y=\left\langle v, u_{8}\right\rangle$, a Sylow 2-subgroup of $X$, then we must have $\left[\widetilde{u_{2} u_{3} u_{5}} \widetilde{u_{6}}, \widetilde{u_{8}}\right] \equiv \alpha\left(\bmod V_{2}\right)($ recall that $E_{1}$ is the $\langle r\rangle$-fixed point space of $\left.R / R_{2}=V / V_{2}\right)$. Taking preimages, this implies $\left[\widetilde{u_{2} u_{3} u_{5} \widetilde{u}_{6}}, \widetilde{u_{8}}\right]=\widehat{u_{4} u_{5}^{2}} \alpha$. We also have $\left[\widetilde{u_{2} u_{3} u_{5}} \widetilde{u_{6}}, \widetilde{u_{6}} \widetilde{u_{8}}\right]=\widehat{u_{4}} \alpha$.

Now, $\rho=\widetilde{u}_{2} \overbrace{3} u_{5} \widetilde{u}_{6}$ has square $\widehat{u}_{4}$ or $\hat{u}_{4} \alpha$, and $\sigma=\widetilde{u}_{6} \widetilde{u}_{8}$ is an involution. Therefore, $(\sigma \rho)^{2}=\sigma^{2} \rho^{2}[\rho, \sigma]=\rho^{2} \hat{u}_{4} \alpha$. Since $\alpha$ has no square root in $G, \rho^{2}=\widehat{u}_{4} \alpha$. Now, $\left[\widetilde{u_{1}^{2}} \widetilde{u_{2}}, \widetilde{u_{6}} \widetilde{u_{7}^{2}}\right]=\widehat{u}_{4}$ (not $\widehat{u}_{4} \alpha$ ) since the arguments lie in $V$. The arguments being involutions, we have, for $\tau=\widetilde{u_{1}^{2}} \widetilde{u_{2}} \widetilde{u}_{6} u_{7}^{2}, \tau^{2}=\widehat{u}_{4}$. So, $(\rho \tau)^{2}=\rho^{2} \tau^{2}[\rho, \tau]=$ $\left(\hat{u}_{4} \alpha\right)\left(\widehat{u_{4}}\right) \cdot 1=\alpha$, a contradiction. Therefore $\alpha \notin Y^{\prime}$, which establishes assertion (i).

We can also see that $\langle\alpha\rangle$ splits off $V$. For $\alpha \notin V_{2}$ and $A V_{2} / V_{2}$ splits off the fixed point free $\langle r\rangle$-submodule, and off $\langle\vec{\rho}\rangle$ as well since $\alpha \notin Y^{\prime}$ and $\rho^{2}=\widehat{u_{4}} \in V_{2}$ can be established by repeating the argument with $\sigma, \rho$, using $[\rho, \sigma]=\hat{u}_{4} \cdot$ In particular $\mho^{1}(V) \subseteq V_{2}$.

Since we know $V_{2}$ and enough about the action of $u_{8}$ on $V / V_{2}$, a corollary is $\left|Y^{\prime}\right|=2^{5}$. In fact $Y^{\prime}=\left\langle V_{2}, x_{1}, x_{2}\right\rangle$, where the $x_{i}$ are suitable elements of the cosets $\widetilde{u}_{6}, \widetilde{u}_{2} \widetilde{u}_{3} u_{5} \widetilde{u}_{6} \widetilde{u}_{7}^{2}$. Another corollary is that $\widehat{u}_{4} a$ has no square root in $\widetilde{G}$. If it did, $\tau^{2}=\widehat{u}_{4} \alpha, \tau \in Y$. Write $\tau=\widetilde{u}_{8} u, u \in V$. Since $\tau^{2} \in A V_{2}, \tau$ must fix $u$ modulo $A V_{2}$; hence $\left[u, u_{8}\right] \in A V_{2} \subseteq C(u)$. So, $\tau^{2}=u_{8}^{2} u^{2}\left[u, u_{8}\right]=u^{2}\left[u, u_{8}\right]$. Since $u^{2} \in V_{2}, \widehat{u}_{4} \alpha=\tau^{2} \in Y^{\prime}$ follows, a contradiction. At this point, (ii) is clear, and so both assertions have been proven. 
For later use, let $\hat{u}_{6}$ denote the above-mentioned suitable element $x_{1} \in u_{6}$; i.e., $\widehat{u_{6}}=\left[\widetilde{u}_{2}, \widetilde{u}_{8}\right]$. Since ${\widetilde{u_{2}}}_{2}, \widetilde{u}_{8} \in C \widetilde{G}^{\left(u_{6}\right.})$, as is easily seen, and $u_{6}$ is conjugate to $u_{4}$, the use of here coincides with the previous meaning for conjugates of $u_{4}$. We now return to $S$ and compute $S_{2}$. Let $L=C_{S}\left(\widehat{u}_{4}\right)=\left\langle S_{2}, \tilde{u}_{2} \tilde{u}_{4}, \widetilde{u}_{3}^{2} \widetilde{u_{3} u_{5}}, \widetilde{u_{8}}\right\rangle$. $|S: L|=2$, and a representative for the nontrivial coset of $L$ in $S$ is $\rho=\widetilde{u}_{4} \widetilde{u}_{5} u_{7} \tilde{u}_{8}$. From our analysis of $\tilde{X}$, we can calculate that $L^{\prime}=\left\langle\widehat{u_{5}^{2}}, \widehat{u_{6}}, \widehat{u_{4}}, \widehat{u_{3}^{2}}\right\rangle$ and $\left|L^{\prime}\right|=$ $2^{4}$. Since $S_{3} \subset L^{\prime} \subset S_{2}, L^{\prime} \triangleleft S$ and $S / L^{\prime}$ has class 2. So, $S_{2}=\left\langle L^{\prime},[\sigma, \rho]\right|$ $\left.\sigma \in\left\{\widetilde{u}_{2} \widetilde{u}_{4}, \widetilde{u_{3}^{2}} \widetilde{u_{3} u_{5}}, \widetilde{u}_{8}\right\}\right\rangle$.

We proceed to determine the $[\sigma, \rho]$. $\left[\tilde{u}_{2} \tilde{u}_{4}, \rho\right]$ is conjugate via $w_{1} w_{8}$ to $\left[\tilde{u}_{16} \widetilde{u}_{2}, \widetilde{u}_{2} \widetilde{u}_{3} u_{5} \widetilde{u}_{6}\right] \in u_{4}$, and the arguments lie in $C\left(\tilde{u}_{4}\right)$. By (i), this conjugate equals $\widehat{u}_{4}$. Conjugating back, $\left[\tilde{u}_{4} \tilde{u}_{6}, \rho\right]=\widehat{u}_{6} \in L^{\prime}$.

Next, define $\tilde{u}_{4}=\left[\widetilde{u_{3}^{2}} \widetilde{u_{3} u_{5}}, \rho\right] \epsilon u_{4}$. Set $\sigma=\widetilde{u_{3}^{2}} \widetilde{u_{3} u_{5}}$. Then, $\sigma^{2}=\widehat{u_{3}^{2}} \widehat{u}_{4} u_{5}^{2}$, as $\sigma \in V$. Also $\rho^{2}=\widehat{u}_{6}$ because $\rho^{w_{1} w_{8}} \in V$ and $\left(\rho^{w_{1} w_{8}}\right)^{2}=\widehat{u}_{4}$. So, $(\rho \sigma)^{2}=$

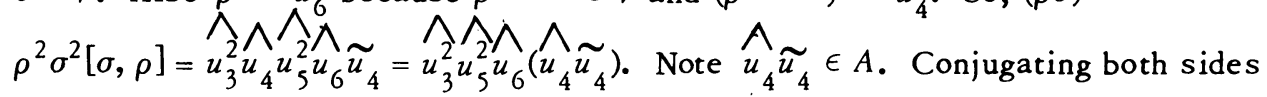
by $\widetilde{u_{3} u_{5}} \widetilde{u_{1}^{2}}=x$, we get $\left(\rho^{x} \sigma^{x}\right)^{2}=\widehat{u}_{6}\left(\hat{u}_{4} \widetilde{u}_{4}\right)$. If $\hat{u}_{4} \neq \widetilde{u}_{4}$, we have a contradiction to (ii). Hence, $\left[\tilde{u}_{3}^{2} \cdot \widetilde{u}_{3} u_{5}, \rho\right]=\hat{u}_{4} \in L^{\prime}$.

This means $S_{2}$ is generated by $L^{\prime}$ (order $2^{4}$ ) and the remaining $[\sigma, \rho]$; viz., $\left[\tilde{u}_{8}, \rho\right] \in u_{7}^{2}$. We conclude $\left|S_{2}\right|=2^{5}=\left|T_{2}\right|$, and that $A=1$ under the hypothesis $A \subseteq S_{2}$.

The remainder of the proof is easy. In our central extension $\tilde{G}$ of $G$ by a 2 group $A$, we have $A \cap S_{2}=1$. Let $\bar{A}$ denote the image of $A$ in $\bar{S}=S / S_{2}$. By the action of $y$ on $T / T_{2}$, Fitting's lemma yields $\bar{S}=[\bar{S},\langle y\rangle] \oplus \bar{A}$, the direct sum as $\langle y\rangle$-modules. Since the element $v$ of order 4 in $E$ normalizes $\langle y\rangle$ and $S, v$ leaves $[\bar{S},\langle y\rangle]$ invariant. Therefore $S_{0}$, the preimage in $S$ of $[\bar{S},\langle y\rangle]$, is a normal subgroup of $U=\langle S, v, A\rangle$, a Sylow 2-subgroup of $\tilde{G}$. Furthermore, $U / S_{0}$ is abelian, since the image is covered by $\langle v, A\rangle$. As $A \cap U^{\prime}=1, A \subseteq Z(\widetilde{G})$, a transfer lemma (2) implies that $A \cap \tilde{G}^{\prime}=1$.

Since $\tilde{G}$ is an arbitrary central extension of $G$ by a 2 -group $A$, we infer $m_{2}(G)=1$.

Showing $m_{p}(G)=1$ for $p \neq 2$ is not hard. Since a Sylow 13-subgroup is cyclic, $m_{13}(G)=1$. Since $G \cap\left(\left\langle u_{1}, u_{9}\right\rangle \times\left\langle u_{5}, u_{13}\right\rangle\right)$ contains a normal subgroup $S$ of order $5^{2}$, which is a Sylow 5-subgroup of $G$, we see that there is an element in $N_{G}(S)$, say $u_{1}^{2} \in G$, which effects a nonspecial transformation on $S$. By (7), $m_{5}(G)=1$. G contains $H=\left\langle u_{8}, u_{16}\right\rangle \times\left\langle u_{4}, u_{12}\right\rangle \cong D_{6} \times D_{6} \cdot H$ contains a normal elementary abelian subgroup $P$ of order 9 . Take $z \in P^{\#} ;\left|C_{G}(z)\right|=2^{2} \cdot 3^{3}$. Let $R$ 
be a Sylow 3-subgroup of $C_{G}(z),|R|=27$. Since $\left|C_{G}(z): R\right|=4$, if $R$ is not normal in $C_{G}(z), C_{G}(z)$ has $A l t(4)$ as a quotient. Since $R$ has no elements of order 9, the extension splits, $C_{G}(z)=K \cdot A l t(4)$ by the Frattini argument. We may assume $z \in K$. But then some involution $i$ of Alt(4) centralizes $K$, a contradiction to $9 \nmid\left|C_{G}(i)\right|$. So, $R \triangleleft C_{G}(i)$. Checking centralizer orders of $3^{\prime}$-elements, $N_{G}(R) / R$ is faithful on $R / \Phi(R)$. If $R$ is elementary abelian, $\left|N_{G}(R) / R\right|=2^{3} \cdot 13$ since all elements of order 3 are conjugate. But this implies 13||$C_{G}(i) \mid$, some involution $i$, impossible. So, $R$ is extra special of order $3^{3},\langle z\rangle=R^{\prime}$. This forces $C_{G}(z) / R \cong Z_{4}$, hence an involution inverts $R / R^{\prime}$, centralizes $R^{\prime}$. By 2.1 of [20], $m_{3}(G)=1$.

This completes the proof that $m(G)=1$.

\section{BIBLIOGRAPHY}

1. J. L. Alperin and D. Gorenstein, The multiplicators of certain simple groups, Proc. Amer. Math. Soc. 17 (1966), 515-519. MR 33 \#1362.

2. N. Bourbaki, Éléments de mathématique. Fasc. XXXIV. Groupes et algèbres de Lie, Actualités Sci. Indust., no. 1337, Hermann, Paris, 1968. MR $39 \# 1590$.

3. H. Cartan and S. Eilenberg, Homological algebra, Princeton Univ. Press, Princeton, N. J., 1956. MR 17, 1040.

4. R. W. Carter, Simple groups and simple Lie algebras, J. London Math. Soc. 40 (1965), 193-240. MR $30 \# 4855$.

5. C. Chevalley, Sur certains groupes simples, Tôhoku Math. J. (2) 7 (1955), 14-66. MR 17, 457.

6. B. Fischer, Finite groups generated by 3-transpositions, Invent. Math. (to appear).

7. D. Gorenstein, Finite groups, Harper \& Row, New York, 1968. MR 38 \#229.

8. R. Griess, Schur multipliers of the known finite simple groups, Bull. Amer. Math. Soc. 78 (1972), 68-71.

9. - Schur multipliers of some sporadic simple groups, J. Algebra (to appear).

10. B. Huppert, Endliche Gruppen I, Die Grundlehren der math. Wissenschaften, Band 134, Springer-Verlag, Berlin, 1967, MR 37 \#302.

1.1. J. Lindsey, On a six dimensional projective representation of $\operatorname{PSU}(4,3)$ (to appear).

12. R. Ree, A family of simple groups associated with the simple Lie algebra of type $\left(F_{4}\right)$, Amer. J. Math. 83 (1961), 401-420; A family of simple groups associated with the simple Lie algebra of type $\left(G_{2}\right)$, Amer. J. Math. 83 (1961), 432-462. MR 24 \#A2617; MR $25 \# 2123$.

13. I. Schur, Über die Darstellung der endlichen Gruppen durch gebrochene lineare Substitutionen, J. Reine Angew. Math. 127 (1904), 20-50; ibid. 132 (1907), 85-137.

14. - Über die Darstellung der symmetrischen und der alternienden Gruppe durch gebrochene lineare Substitutionen, J. Reine Angew. Math. 139 (1911), 155-250.

15. R. Steinberg, Générateurs, relations, et revêtements de groupes algébriques, Colloq. Théorie des Groupes Algébriques (Bruxelles, 1962), Librarie Universitaire, Louvain; Gauthier-Villars, Paris, 1962, pp. 113-127. MR 27 \#3638. 
16. R. Steinberg, Representations of algebraic groups, Nagoya Math. J. 22 (1963), 33-56. MR $27 \# 5870$.

17. - Variations on a theme of Chevalley, Pacific J. Math. 9 (1959), 875-891. MR $22 \# 79$.

18. - Lectures on Chevalley groups, Yale University Notes, New Haven, Conn., 1967.

19. J. Tits, Algebraic and abstract simple groups, Ann. of Math. (2) 80 (1964), 313329. MR $29 \# 2259$.

20. D. Wales and J. McKay, The multipliers of the simple groups of order 604,800 and 50, 232, 960, J. Algebra 17 (1971), 262-272. MR $43 \# 340$.

21. H. N. Ward, On the triviality of primary parts of the Schur multiplier, J. Algebra 10 (1968), 377-382. MR 38 \#1181.

22. K. Yamazaki, On projective representations and ring extensions of finite groups, J. Fac. Sci. Univ. Tokyo 10 (1964), 147-195. MR 31 \#4842.

DEPARTMENT OF MATHEMATICS, UNIVERSITY OF MICHIGAN, ANN ARBOR, MICHIGAN 48104 